Florida International University FIU Digital Commons

\title{
Inspection of Parts with Complex Geometry and Welds with Structural Health Monitoring Techniques
}

Srikanth Korla

Florida International University, skorl001@fiu.edu

DOI: $10.25148 /$ etd.FI1 1081003

Follow this and additional works at: https://digitalcommons.fiu.edu/etd

\section{Recommended Citation}

Korla, Srikanth, "Inspection of Parts with Complex Geometry and Welds with Structural Health Monitoring Techniques" (2011). FIU Electronic Theses and Dissertations. 460.

https://digitalcommons.fiu.edu/etd/460 


\section{FLORIDA INTERNATIONAL UNIVERSITY}

Miami, Florida

INSPECTION OF PARTS WITH COMPLEX GEOMETRY AND WELDS WITH STRUCTURAL HEALTH MONITORING TECHNIQUES

A dissertation submitted in partial fulfillment of the

requirements for the degree of

DOCTOR OF PHILOSOPHY

in

MECHANICAL ENGINEERING

by

Srikanth Korla

2011 
To: Dean Amir Mirmiran

College of Engineering and Computing

This dissertation, written by Srikanth Korla, and entitled Inspection of Parts with Complex Geometry and Welds with Structural Health Monitoring Techniques, having been approved in respect to style and intellectual content, is referred to you for judgment.

We have read this dissertation and recommend that it be approved.

$\begin{array}{r}\text { Sabri Tosunoglu } \\ \hline \text { Yiding Cao } \\ \hline \text { Jean Andrian } \\ \hline \text { Ibrahim Tansel, Major Professor }\end{array}$

Date of Defense: June 15, 2011

The dissertation of Srikanth Korla is approved.

Dean Amir Mirmiran

College of Engineering and Computing

Dean Kevin O'Shea

University Graduate School

Florida International University, 2011 
(C) Copyright 2011 by Srikanth Korla

All rights reserved. 


\section{DEDICATION}

I would like to dedicate this dissertation to my family: my grandfather Late Vishwanatha Rao Gunta and my grandmother Kundanam, My parents Sriramulu Korla and Bharati Gunta, and my ever caring brother Rajesh Korla; without whose support and endless love, the completion of the work would not have been possible. 


\section{ACKNOWLEDGMENTS}

First of all, I would like to express my profound appreciation to my major professor, Dr. Ibrahim Tansel, for his great care and guidance to successfully finish my Doctoral degree. I also thank my dissertation committee member, Dr. Sabri Tosunoglu for his valuable suggestions. I wish to thank my other dissertation committee members, Dr. Yiding Cao and Dr. Jean Andrian for their help and mentorship throughout my dissertation. I would also like to thank Dr. Cesar Levy and Dr. George S Dulikravich for their perpetual care and financial support to finish my PhD successfully.

I would like to extend my sincere thanks to my post doctorial research fellow member, Dr. Mustafa Demetgul for initiating motivation in me. I heart fully thank fellow lab mates and friends, Mr.Gurjashan Singh, Mr. Gurjiwan Singh and Ms. Minig Li for their valuable support at mechatronics laboratory.

I would like to extend my thanks to all my colleagues and friends for those numerous times they helped me out with my work and encouragement. Especially the assistance offered by Mr. Sudheer Posireddy and care taken by my best friend Mr. Srinivas Boddepalli was exceptionally great. I also express my sincere thanks to my friends Mr. Puneet Gill, and Mr. Chandu for their support at FIU. Last but not the least I would like to thank my beautiful wife Swapna Boddepalli for her love and care showed towards finishing my PhD successfully.

Finally, I would like to thank Florida International University Mechanical and Material Engineering department for giving me the opportunity to pursue the most prestigious $\mathrm{PhD}$ degree. 


\section{ABSTRACT OF THE DISSERTATION \\ INSPECTION OF PARTS WITH COMPLEX GEOMETRY AND WELDS WITH STRUCTURAL HEALTH MONITORING TECHNIQUES}

by

Srikanth Korla

Florida International University, 2011

Miami, Florida

\section{Professor Ibrahim Tansel, Major Professor}

Structural Health Monitoring (SHM) systems were developed to evaluate the integrity of a system during operation, and to quickly identify the maintenance problems. They will be used in future aerospace vehicles to improve safety, reduce cost and minimize the maintenance time of a system. Many SHM systems were already developed to evaluate the integrity of plates and used in marine structures. Their implementation in manufacturing processes is still expected. The application of SHM methods for complex geometries and welds are two important challenges in this area of research.

This research work started by studying the characteristics of piezoelectric actuators, and a small energy harvester was designed. The output voltages at different frequencies of vibration were acquired to determine the nonlinear characteristics of the piezoelectric stripe actuators. The frequency response was evaluated experimentally. AA battery size energy harvesting devices were developed by using these actuators. When the round and square cross section devices were excited at $50 \mathrm{~Hz}$ frequency, they generated $16 \mathrm{~V}$ and $25 \mathrm{~V}$ respectively. 
The Surface Response to Excitation (SuRE) and Lamb wave methods were used to estimate the condition of parts with complex geometries. Cutting tools and welded plates were considered. Both approaches used piezoelectric elements that were attached to the surfaces of considered parts. The variation of the magnitude of the frequency response was evaluated when the SuRE method was used. The sum of the square of the differences was calculated. The envelope of the received signal was used for the analysis of wave propagation.

Bi-orthogonal wavelet (Binlet) analysis was also used for the evaluation of the data obtained during Lamb wave technique. Both the Lamb wave and SuRE approaches along with the three methods for data analysis worked effectively to detect increasing tool wear. Similarly, they detected defects on the plate, on the weld, and on a separate plate without any sensor as long as it was welded to the test plate. 


\section{TABLE OF CONTENTS}

CHAPTER PAGE

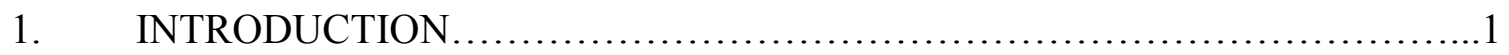

1.1 Structural Health Monitoring (SHM) ......................................

1.2 Scope of Research.........................................................

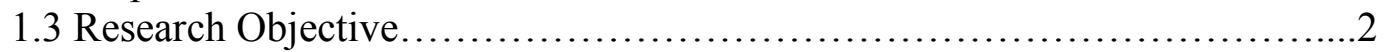

1.4 The Dissertation Outline.............................................. 3

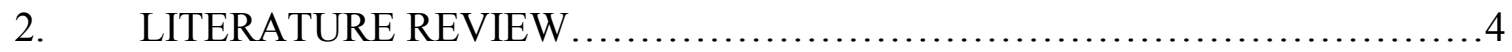

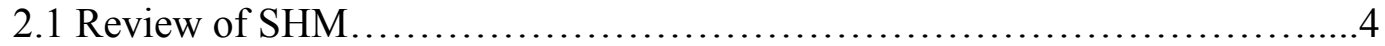

2.2 Piezoelectric Materials.................................................... 5

2.3 SHM using Piezoelectric Materials............................................. 8

2.3.1 Electro Mechanical Impedance Method..............................9

2.3.2 Guided Surface Waves...........................................11

2.4 Methods for Data Analysis in SHM....................................

2.4.1 Wavelet Transformation.......................................................16

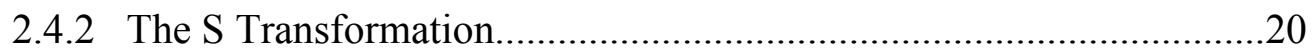

2.5 Monitoring Tool Wear in Machining......................................20

2.6 Brief Summary of the Chapter.......................................24

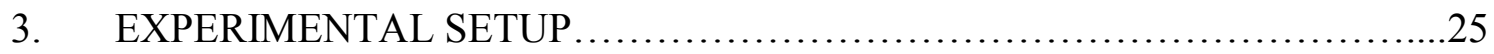

3.1 Experimental Setup to Investigate Electrical Response.....................25

3.2 Experimental Setup to Inspect Complex Geometry Parts and welds.............30

3.2.1 Monitoring Wear of an End Mill..........................................30

3.2.2 Detecting Imperfections in Welds.................................34

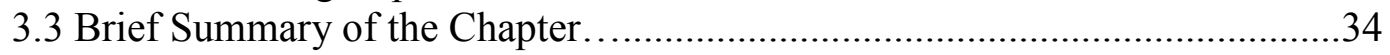

4. PROPOSED PROCEDURES FOR DATA ANALYSIS ........................36

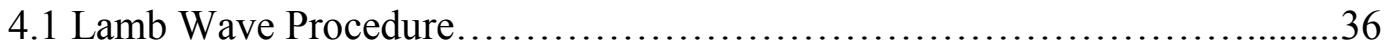

4.2 Surface Response to Excitation (SuRE) Technique..........................39

4.3 Bi-orthogonal Wavelet (Binlet) Analysis ................................40

4.4 Brief Summary of the Chapter........................................ 44

5. RESULTS AND DISCUSSION: CHARACTERIZATION OF PIEZOELECTRIC MATERIALS AND ENERGY SCAVENGING...............45

5.1 Frequency Response and Nonlinear Characterization..........................46

5.2 Electrical Response due to Harmonic Excitation of Actuators..................50

5.3 Brief Summary of the Chapter............................................53 
6. RESULTS AND DISCUSSION: IDENTIFICATION OF DEFECTS OF COMPLEX SHAPES AND WELDS ....................................54

6.1 Wear Estimation of an End mill........................................54

6.1.1 Lamb wave Analysis...........................................54

6.1.2 Analyzing the Surface Response data.........................67

6.1.3 Binlet Analysis..................................................76

6.2 Classification of Imperfections in Weld Joints..........................8 80

6.2.1 Lamb wave Analysis..........................................80

6.2.2 Data Analysis with SuRE Approach..............................84

6.2 .3 Binlet analysis............................................. 89

6.3 Brief Summary..................................................91

7. CONCLUSION AND FUTURE WORK...................................94

7.1Characterization of Piezoelectric Materials and their Application..................94

7.2 Monitoring the Integrity of the Complex Geometries and Welded Parts.......95

7.3 Future Work.....................................................96

LIST OF REFERENCES..................................................97

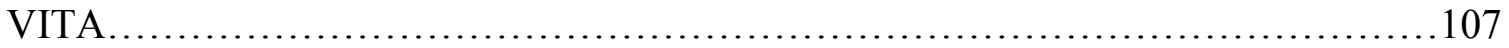




\section{LIST OF FIGURES}

FIGURE

PAGE

Figure 2.1 Comparison of Symmetric and Anti-Symmetric wave dispersion curves (lowest modes). .15

Figure 2.2 Shifting and Scaling operations during wavelet decomposition.....

Figure 2.3 Perfectly reconstructed orthogonal filter

bank.

Figure 3.1 Schematic diagrams representing the experimental setup that evaluated frequency response.

Figure 3.2 Experimental setup used to investigate the electrical response and nonlinear characteristics of piezoelectric actuators.

Figure 3.3 Comparison of the electrical response testing devices

(Round, and Rectangular shapes). .28

Figure 3.4 Circuit diagram and simulation to convert AC output to DC: (a) Rectifying circuit (b) Simulation of the circuit to determine DC output voltage....

Figure 3.5 Experimental setup that classified end mill tool wear using wave method (Métis Design SHM Node MD 7502).

Figure 3.6 Experimental setup used to record the surface response to excitation signals

Figure 3.7 Sensor attachments and wear on the end mill specimens.... .33

Figure 3.8 Butt weld joint with a $1 / 4$ th inch cut and $1 \mathrm{~mm}$ diameter hole.

Figure 4.1 Simulation of wave modes (Excitation frequency: $680 \mathrm{kHz}$ )

Figure 4.2 Data analysis using wave Technique 38

Figure 4.3 SuRE Technique to detect damages on a weld joint. ...

Figure 4.4 Decomposition and Reconstruction filters of a bi-orthogonal wavelet $\left(\mathrm{N}_{\mathrm{d}}=7, \mathrm{~N}_{\mathrm{r}}=9\right)$.

Figure 5.1 Frequency spectrum of single piezoelectric actuator without any weight......46 
Figure 5.2 Frequency spectrum of a single piezoelectric actuator with half the weight $(2.85 \mathrm{~g})$

Figure 5.3 Non Linear output characteristics of piezoelectric actuators..................48

Figure 5.4 Comparisons of RMS and Peak-Peak output values.........................49

Figure 5.5 Electrical response of the energy harvester (Round shape).................50

Figure5.6 Electrical response of the energy harvester (Rectangular design) ...............51

Figure 5.7 Comparison of Volume and Power Density of energy harvesting devices.....53

Figure 6.1 Comparison of Data 1 (no wear signals) with Data 2:

(a) Slightly worn (b) Medium wear(c) Heavy wear (d) Severe wear

(e) Extreme wear of mill tool. .56

Figure 6.2 Comparison of S Transformation data zoomed below $600 \mathrm{KHz}$ (a) No wear (b) Slightly worn (c) Heavy wear (d) Extreme wear

Figure 6.3 Comparison of s-transformation of Micro tool (a) No wear

(b) Slightly worn (c) Heavy wear (d) Extreme wear

Figure6.4 Comparison of s-transformation envelopes during five stages of wear

(a) Slightly worn (b) medium wear (c) Heavy wear (d) Severe Wear

(e) Extreme wear of mill tool

Figure 6.5 Comparison of s-transformation envelopes of the micro tool

(a) Slightly worn (b) medium wear (c) Heavy wear (d) Severe Wear

(e) Extreme wear of mill tool

Figure 6.6 Simulink model to evaluate square of difference value......................66

Figure 6.7 Simulation square of difference data using Simulink model .................67

Figure 6.8 Frequency responses at the receiving sensor (a) Data1: No wear

(b) Data 2-Data 6: Slightly worn -extreme wear of the tool

Figure 6.9 Comparison of sum square of difference among wear levels of the End mill 68

Figure 6.10 Comparison of sum square of difference among wear levels (Micro tool) 
Figure 6.11 Comparison of sum square of difference (Dissimilar frequency intervals).

Figure 6.12 Comparison of sum square of difference for Micro tool (dissimilar frequency intervals).

Figure 6.13 Comparison of the sum square differences at different bins .72

Figure 6.14 Comparison of peak data of the envelopes (a) No wear vs. slightly worn (b) No wear vs. medium wear (c) No wear vs. heavy wear

(d) No wear vs. severe wear (e) No wear vs. extreme wear. .75

Figure 6.15 Comparison of peaks at $20.4 \mathrm{kHz}$ frequency and $90 \mathrm{kHz}$ frequency at

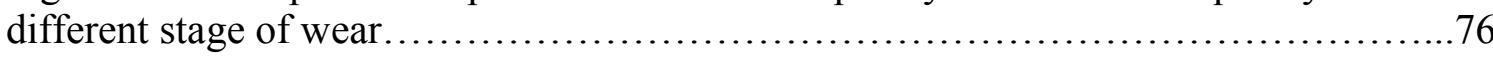

Figure 6.16 Binlet Coefficients: (a) no wear (b) slightly worn (c) medium wear (d) heavy wear..... .77

Figure 6.17 Comparison Residuals (SD): (a) No wear (b) Slightly worn (c) medium wear (d) heavy wear.

Figure 6.18 Comparison Residuals (Mean Abs. Deviation): (a) No wear (b) Slightly worn (c) medium wear (d) heavy wear.................................... 79

Figure 6.19 Comparison of SD residuals at three different levels of binlet analysis.......80

Figure 6.20 Comparison perfect signal data (Data 1) with signals with Imperfections (Data2): (a) $1 / 4^{\text {th }}$ inch cut

(b) 0.0625 inch diameter hole (Lamb wave data).

Figure $6.21 \mathrm{~s}$-transformation data (Zoomed below $600000 \mathrm{~Hz}$ )

(a) Initial Signal (b) Cut (c) Hole.

Figure6.22 Comparison of s-transformation envelopes ( $200 \mathrm{kHz})$ between a

(a) $14^{\text {th }}$ inch cut (b) 0.0625 inch diameter drilled hole.

Figure 6.23 Comparison (a) perfect signal data (Data 1) with signals with Imperfections (Data2): (b) $1 / 4^{\text {th }}$ inch cut (c) 0.625 inch diameter hole (SuRE data)......84

Figure 6.24 Comparison of amplitude difference between perfect weld (data 1) and (a) cut (b) hole. .85

Figure 6.25 Comparison of square of difference between perfect weld (Data 1) and Cut (Data 2) and Hole (Data 3)..........................86 
Figure 6.26 Comparison of sum square of difference between

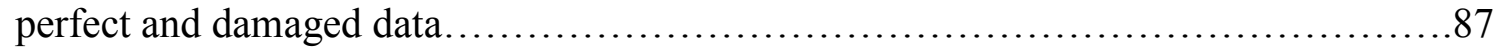

Figure 6.27 Comparison of sum square of difference between perfect and weld with Cut and Hole at dissimilar frequency intervals....................87

Figure 6.28 Comparison of Sum square of difference at different bins..................88

Figure 6.29 Binlet coefficients (a) $1 / 4$ inch cut

(b) 0.0625 inch diameter drilled hole (D1 level decomposition) .......................90

Figure 6.30 Comparison of statistical data between a the cut and hole imperfections: (a) Standard Deviation (b) Mean Abs Deviation.............92

Figure 6.31 Error in SD values between different trails of the experimental analysis.....96 


\section{ABBREVIATIONS}

$\begin{array}{ll}\text { SHM } & \text { Structural Health Monitoring } \\ \text { PWAS } & \text { Piezoelectric Wafer Active Sensors } \\ \text { QP } & \text { Quick-Pack } \\ \text { MFC } & \text { Micro-Fiber Composite } \\ \text { VTS } & \text { Vibration Test System } \\ \text { FFT } & \text { Fast Fourier Transform } \\ \text { STFT } & \text { Short Time Fourier Transform } \\ \text { CWT } & \text { Continuous Wavelet Transform } \\ \text { DWT } & \text { Discrete Wavelet Transform } \\ \text { APC } & \text { American Piezo Corporation } \\ \text { FEM } & \text { Finite Element Model } \\ \text { GONNS } & \text { Genetically Optimized Neural Network Systems } \\ \text { SRS } & \text { Standard Research Systems } \\ \text { SD } & \text { Standard Deviation }\end{array}$




\section{CHAPTER 1}

\section{INTRODUCTION}

\subsection{Structural Health Monitoring (SHM)}

Structural Health Monitoring (SHM) is developed for continuous monitoring of the condition of the structures. Unlike conventional scheduled driven inspection, SHM allows condition based maintenance; it improves reliability and safety while reducing the maintenance cost. Many researchers are expecting wide applications of the SHM systems in the aerospace structures in future.

SHM techniques mainly use two approaches: (a) Passive and (b) Active method. Passive SHM systems have only sensors. They monitor the stress, acceleration and temperature, when the system is excited externally. Active SHM systems excite the system and monitor the response at the same time. One of the applications of active SHM employs piezoelectric elements, and monitors the damages by creating Lamb waves. The damage identification characteristics of piezoelectric materials are presented in Chapter Six of this dissertation.

\subsection{Scope of Research}

The SHM methods have been developed in last two decades, mainly by the civil engineering and aerospace communities. Aerospace applications estimated location, severity and type of damage mainly in metal and composite plates. The goal of this study is to adopt SHM methods into other manufacturing applications. This dissertation focused on evaluation of the integrity of weld joints and cutting tools. 


\subsection{Research Objective}

The recent developments of continuous SHM using piezoelectric materials have opened new approaches for active damage recognition techniques in thin plates, and complex geometries of metallic structures. The present research investigates the capabilities of piezoelectric actuators and their application in SHM. The proposed methodologies and newly developed analytical techniques in this dissertation are listed briefly as follows:

(1) Determining the dynamic characteristics and electrical output of piezoelectric materials.

(2) Proposing an effective wear monitoring methodology to estimate the progressive wear in an end mill tool using Lamb wave and Surface Response to Excitation (SuRE) techniques.

(3) Implementing experimental procedures and signal processing techniques to identify dissimilar imperfections in welds.

(4) Developing binlet filters, to analyze the damage detection characteristics using statistical residue coefficients and wavelet analysis

Condition monitoring during machining is vital to avoid unwanted interruptions of manufacturing processes. The cutting tool wear was monitored earlier using force and torque signals (Teti et al, 2010) and other methods like vibration techniques, laser techniques and Acoustic methods (Byrne et. al, 1995). The objective of the present work is to investigate the performance of SHM methods in cutting tools and welds, using the piezoelectric materials as sensors. 


\subsection{The Dissertation Outline}

The dissertation is organized into Seven Chapters. Chapter Two outlines the detailed studies on related topics of the present research. It describes the previous studies about the characterization of piezoelectric actuators and sensors, bonding characteristics, surface waves and signal processing tools. It also outlines various techniques that use SHM methods for aerospace applications. The experimental setup and data collection methods are presented in Chapter Three. The Procedure followed to analyze the experimental data is elaborated in Chapters Four. Chapter Five discusses the results and the electricity generation characteristics of the piezoelectric elements. The results of damage estimation for welds and tools are outlined in Chapter Six. Chapter Seven summarizes important conclusions and scope for extended research. 


\section{CHAPTER 2}

\section{LITERATURE REVIEW}

As outlined in the previous chapter, this dissertation work investigates the electric performance, and evaluates imperfections in metallic structures with complex geometry by using piezoelectric materials. Therefore, the literature related to the previous SHM techniques, and damage identification methodologies using piezoelectric materials is elaborately discussed in the following sections of this chapter. The theory behind related signal processing tools is also reviewed thoroughly in the following sections. The Chapter presents the literature survey behind the wavelet theory. The support of the literature resources to establish the present work is briefly discussed at the end of the chapter.

\subsection{Review of SHM}

The interest to monitor structural damage at its earliest possible stage is very important in civil architectures, aerospace and mechanical systems. SHM technology involves the observation of a system over a time using periodically sampled dynamic response from an array of sensors. Farrar and Doebling (1996) described the SHM problem as a four part process: 1. the operational evaluation process defines the damage of a system that is being monitored, 2. data acquisition techniques determine the type and number of sensors, location of the sensor, the data recording and transmission hardware, 3. feature extraction methods distinguish undamaged and damaged structures by comparing the damage sensitive properties, 4. statistical models were developed for 
feature discrimination by quantifying the damage level of the structure. Structural health monitoring have been an emerging tool that uses vibration characteristics of the structure to identify the internal damages in structures like airframes, vessels, bridges, and machinery (Giurgiutiu, 2001; Mehrjoo et al., 2008). The applications of SHM and various type of sensors used during SHM are tabulated in Table 2.1. Piezoelectric materials have been widely used as sensors to monitor the structural health during the data acquisition phase of SHM.

Table 2.1 Applications of SHM and Sensor Technology

\begin{tabular}{|l|l|}
\hline Applications & Sensor Technology \\
\hline $\begin{array}{l}\text { Structural Integrity of Buildings and civil } \\
\text { engineering structures }\end{array}$ & Fiber optical sensors \\
& FBG (Fiber Brag Grating) sensors \\
Health monitoring of composite plates & Carbon Fiber/ Nanotube \\
Crack and damage detection Air structures & Based sensors \\
& Piezoelectric sensors \\
& Magneto- elastic active sensors \\
\hline
\end{tabular}

\subsection{Piezoelectric Materials}

Many researchers worked on conversion of mechanical energy to the electricity (Mossi et al., 2005; Kim et al., 2005; Minazara et al., 2006), Piezoelectric materials have been an excellent choice for conversion of the electrical energy to mechanical energy and vice versa. The different types of piezoelectric materials includes Lead Zirconate Titanate (PZT), Quick Pack (QP), monolithic piezo ceramic materials embedded in the epoxy 
matrix, and Micro-Fiber Composite (MFC) (Goldfarb and Jones, 1999; Sodano et al., 2005). They have found many engineering applications and used for manufacturing of the sensors and actuators (Niezrecki et al., 2001; Sirohi and Chopra, 2001). They are inexpensive, robust, and conveniently adaptable in most of the applications.

Piezoelectric materials can be used as sensors and actuators. As sensors they are used to convert mechanical energy to electrical energy. They convert electrical energy to mechanical vibration when they are used as exciters or actuators. Giurgiutiu et al., (2004, 2008), cited the constituent equations of piezoelectric materials by deriving mechanical strain $\left(S_{i j}\right)$ and electrical displacement $\left(D_{j}\right)$ in terms of mechanical stress $T_{k l}$ and electric field $E_{k}$ :

$$
\begin{aligned}
& S_{i j}=S_{i k l}^{E} T_{k l}+d_{k i j} E_{k} \\
& D_{j}=d_{j k l} T_{k l}+\varepsilon_{j k}^{T} E_{k}
\end{aligned}
$$

$S_{i k l}^{E}, d_{k i j}$, and $\varepsilon_{j k}^{T}$ are mechanical compliance at zero electric field, piezo electric coupling between electrical and mechanical variables and dielectric permittivity measured at zero mechanical stress respectively.

The piezoelectric stripe actuators resemble cantilever beams and previously an aluminum beam with piezoelectric patches (Sodano, 2003), a bimorph beam (Erturk, 2009) and a plate (Charnegie, 2007) were modeled. The analytical model of bimorph beams for energy harvesting has been discussed and the expression of stiffness for a horizontal cantilever beam against the vertical forces acting at the tip of a bimorph beam 
was derived by Erturk, 2009 is described in eq. (2.3) based on the lumped-parameter model and when the base was subjected to a harmonic excitation:

$$
k_{e q}=\frac{3 Y I}{L^{3}}
$$

$Y I$, is the bending stiffness of the beam of length L. The un-damped natural frequency depends on the equivalent stiffness $\left(k_{e q}\right)$ and equivalent mass $\left(m_{e q}\right)$, and derived from eq. (2.3):

$$
\omega_{n}=\sqrt{\frac{k_{e q}}{m_{e q}}}=\sqrt{\frac{3 Y I / L^{3}}{\left(\frac{33}{140}\right) m L+M_{t}}}
$$

$m$, is the mass per unit length of the beam. $M_{t}$, is the mass at the tip of the beam. The analytical model for the undamped natural frequency $\left(\omega_{n}\right)$ of a uniform cantilever (clamped-free) plate with no additional mass was derived by Charnegie (2007) with the following expression:

$$
\omega_{n}=\lambda_{n} \sqrt{\frac{D}{\rho h b a^{3}}}
$$

The characteristic values $\left(\lambda_{n}\right)$ for the first 3 modes of a square cantilever plate their values are $3.494,8.54$ and 21.44. The parameters $h, a$ and $b$ represent the thickness, length and width of the beam respectively. The modulus of elasticity $(D)$ was calculated using the following equation (2.6):

$$
D=\frac{E t^{3}}{12\left(1-v^{2}\right)}
$$


The modulus, thickness and Poisson's ratio of the material were represented byE, $t$ and $v$ respectively. Studies were available on the modeling, testing and performance of the energy harvesters (Lu et al., 2004; Dutoit et al., 2005; Twiefel et al., 2006; Beeby et al., 2006). The energy harvesters that fit into small spaces or standard shapes such as standard AA battery size were developed in mechatronics laboratory (Tansel et al., 2008).

Degradation evaluation of piezoelectric sensors was performed recently by many researchers. A simple strain based SHM approach adopted to monitor born/ epoxy patch repair that could overcome the limitations in using the conventional strain gauges for SHM. These approaches use the Bragg grating optical fibers as strain gauges and helps in patch repair. Rodrigo et al. (2009) demonstrated how the strain based methodology can be utilized to detect the crack propagation in secondary bonded woven E-glass/vinyl ester composite double plate joints (Baker et al., 2008).

The effectiveness of sensor bonding with its host structure was estimated by Giurgiutiu, 2008 and Baker et al., 2008. The deboning and crack defects of the piezoelectric elements were identified under varying structural load conditions; the change in capacitive values of the piezoelectric materials during the de-bonding of the sensors with their host structures was estimated earlier by Park et al., 2006; Sohn and Lee, 2009. Piezoelectric sensor characteristics are estimated at various physical and mechanical environments, and the reliability of the adhesives used to bond these patches was monitored. The interfacial shear stress behavior between the piezoelectric sensors and the host structure under the presence of two or more surface waves was estimated by Pfeiffer et al., 2007; Rajic et al., 2002. 


\subsection{SHM using Piezoelectric Materials}

Firstly Giurgiutiu (2003) and Lynch (2004) used piezoelectric Ultrasonic SHM system for an aircraft wing inspection. The low cost and light weight piezoelectric disc transducers were bonded to various parts of an aircraft wing for detection, location and growth monitoring of defects (Zhao et al., 2007). A real-time method for predicting accurate damage location in complex structures was proposed to provide a way to efficient damage location detection for large scale complex structures, with a minimum number of sensors. Piezoelectric smart materials embedded in laminated composite structures evaluated the intensity of damage. Past work also described the in-service inspection of composite inaccessible structures without additional excitation facility. (Kessler and Raghavan, 2009; Yan and Yam, 2002).

Piezoelectric wafer active sensors (Lin et al., 2006), developed for monitoring fatigue cracks and corrosion damage, were often used as embedded ultrasonic transducers on metallic frames. Conventional ultrasonic transducers work with surface tapping and pressure to the objects surface, whereas PWAS act through pinching with the object's surface (Su et al., 2006). Giurgiutiu, 2003 described the effectiveness of piezoelectric wafer active sensors during health monitoring of metallic slats. The SHM using piezoelectric materials as sensors was mainly performed by two methods called the Electromechanical Impedance and Lamb wave technique.

\subsubsection{Electro Mechanical Impedance Method}

Damage in a structure can be indicated by monitoring the changes in electro mechanical impedance. High frequency structural excitations through surface bonded 
piezoelectric transducers were used by Park and Inman (2006). A spectral element method was used by Peairs et.al., 2007, in combination with electric circuit analysis, for impedance modeling. Grisso and Inmann (2009) developed a sensor diagnostics method to identify the unhealthy sensors during the impedance based SHM. A digital wideband technique was proposed by Kim et al., to reduce the power dissipation during the impedance based SHM systems.

Giurgiutiu (2008) described his experiments conducted using electro-mechanical impedance on durability and survivability of PWAS. E/M impedance technique was used to characterize the damage behavior in a truss, beam and a concrete tube and the electromechanical signature at the piezoelectric path surface was analyzed to identify the damage (Lim et al., 2006). A two dimensional electro mechanical impedance model was proposed to predict the electrical admittance change on the PZT transducer due to damage. Nonlinear feature damage identification technique using self-sensing impedance measurements was developed earlier by Rutherford et al. (2007). The applicability of the nonlinear feature for damage identification was investigated in various frequency ranges using impedance signals measured form a laboratory test impedance methods. Piezoelectric self-diagnosis schemes were developed to detect the functionality and defects within the piezoelectric transducers. Pulse echo method and acoustic ultrasonic methods were used for structural interrogation at far field, and E/M impedance method was more appropriate technique used to detect the damages in near fields (Giurgiutiu, 2002 and 2003). 
Baptista and Filho (2009) in their article described the influence of structural area on the performance of SHM systems based on electro mechanical Impedance (E/M Impedance), and it was observed that the sensitivity of piezoelectric transducers for damage detection significantly decreases with the mechanical impedance of the host structure.

\subsubsection{Lamb wave based methods:}

Surface waves are used in the palates to determine the damages in plates and tubes using piezoelectric sensors and actuators. The surface wave techniques are reviewed in the following paragraphs. Lee and Staszewski (2007) analyzed a fatigue crack on an aluminum plate using Lamb waves to recognize the optimal location of the sensor.

Guided waves find a way to overcome the drawback discussed in previous section that uses E/M Impedance method. Guided waves have a great significance in structural health monitoring in recent years as they can travel large distances in plates and pipes with very minimum energy loss. Giurgiutiu (2008) in his well-known text book on SHM, Lee and Staszewski (2003), Pfeiffer (2007) have described the potentiality of Lamb waves between the piezoelectric sensors in plates and air structures. The performance of acoustic ultrasonic waves in damage detection was demonstrated by analyzing two dimensional wave interactions with slot type defects. The change in the output of the guided ultrasonic waves was able to determine the damage in the structure. The response of the ultrasonic transient waves was investigated as a function of different defect sizes of cracks. The strain coupled surface bonding of the piezoelectric sensors enable effective 
transmission of in-plane ultrasonic Lamb waves. Theoretical models and experiments results were previously presented by Giurgiutiu since 2001 indicated that piezoelectric sensors can satisfactorily perform Lamb wave transmission and reception in aluminum and metallic plates. A comprehensive review of Lamb wave based damage identification techniques for composite materials was established by scholars like Su and Lin (2006), who elaborately discussed the physics behind the Lamb wave propagation in laminated composites. A separate work by Lin and Giurgiutiu (2006) demonstrated that the flexible piezoelectric materials like piezoelectric polymers perform the same Lamb wave transmission and reception functions as piezoelectric wafer active sensors perform.

Grabowska et al. (2008) demonstrated the propagating Lamb waves to distinguish different kinds of failure occur during structural health monitoring, Park et al. (2006) and Rajic et al. (2002) used Lamb waves in a beam to analyze and characterize the degradation performance.

Time of flight estimation methods like cross-correlation, envelop moment, matching pursuit decomposition, and dispersion compensation were compared using a dispersive Lamb wave mode (Xu et al., 2009). A solution for generation and propagation of Lamb waves in homogeneous isotropic viscoelastic plates was established by Vonende et al. (2009). This methodology for analytical analysis of Lamb wave generation and propagation was identified as an effective technique when the wave guide exhibits significant material damping.

Experimental results were presented by Huang et al. (2008) and Tan et al. (1995) on directionality of Lamb waves. An approach was developed using the fundamental 
symmetric mode of Lamb waves to detect the delamination in unidirectional fiber composite materials, and it was observed that the Lamb wave amplitude decreases significantly over a delamination region. The wave displacement fields were evaluated and the displacement fields excited by a piezo patch actuators were found to be directional; the directionality of the surface wave displacement fields were governed by the bending deformation of the piezoelectric actuator.

Development of theoretical models for the surface bonded piezoelectric sensors and modeling of Lamb waves was performed in earlier research. Dunn et al. (2009) presented an analytical model to couple transducer dynamics with axi-symmetric guided surface wave excitation for isotropic plates. Piezoelectric patch actuators work as a point source that excites an axi-symmetric wave motion. It was proved in Huang et al. (2009) that the Lamb wave displacement fields generated by piezoelectric patches are directional and frequency dependent. Asymmetric Lamb wave propagation was modeled based on superposition of plate bending modes by Kerkira et al. (2007).

Zhongqing et al. (2006), Raghavan and Cesnik (2005) developed a mathematical model to describe guided wave structural health monitoring. The size of the sensors was optimized based on numerical analysis. Park et al. (2009) investigated the effect of sensor and bonding defects of Impedance models using single degree of freedom spring mass damper system.

The equation of motion for isotropic elastic medium for a plate with stress free upper and lower, and in cylindrical pipes were derived by Silk and Bainton (1979), Rose et al. (1994). If $\rho$ is density of the metal structure and $\lambda$ and $\mu$ are lame's constants, then 
the corresponding equations of motion due to a displacement field $(u)$ are given by eq.(2.7) and eq.(2.8):

$$
\begin{aligned}
& \text { Plate Equation: } \mu \nabla^{2} u+(\lambda+\mu) \nabla \nabla \cdot u=\rho \frac{\partial^{2} u}{\partial t^{2}} \\
& \text { Pipe Equation: } \mu \nabla u+(\lambda+\mu) \nabla \nabla \cdot u=\rho \frac{\partial^{2} u}{\partial t^{2}}
\end{aligned}
$$

Giurgiutiu (2008) simulated Lamb wave speed dispersion curves (Figure 2.2) in plates from the Rayleigh-Lamb equation. The governing equations with a longitudinal and transverse wave speeds, $c_{p}=\sqrt{\frac{(\lambda+2 \mu)}{\rho}}$ and $c_{s}=\sqrt{\frac{\mu}{\rho}}$ are described using eq. (2.9) to eq.(2.12):

$$
\begin{gathered}
A\left(-c_{3} \sin \alpha d\right)+H\left(c_{4} \sin \beta d\right)=0 \\
A\left(c_{1} \cos \alpha d\right)+H\left(c_{2} \cos \beta d\right)=0 \\
B\left(c_{1} \sin \alpha d\right)+G\left(-c_{2} \sin \beta d\right)=0 \\
B\left(c_{3} \cos \alpha d\right)+G\left(c_{4} \cos \beta d\right)=0 \\
\alpha=\sqrt{\left(\frac{\omega^{2}}{c_{p}^{2}}-\xi^{2}\right)} ; \quad \beta=\sqrt{\left(\frac{\omega^{2}}{c_{s}^{2}}-\xi^{2}\right)} \\
c_{1}=(\lambda+2 \mu) \alpha^{2}+\lambda \xi^{2}, c_{2}=2 i \mu \xi \beta, c_{3}=2 i \xi \alpha, c_{4}=\xi^{2}-\beta^{2}
\end{gathered}
$$

$A, H, B, G$ were determined by the stress free conditions at the upper and lower surface of the plate. These coefficients would correspond to the symmetric and antisymmetric Lamb waves. 
If the product of Frequency (f) and the thickness (d) is equal to zero, then both symmetric and anti-symmetric waves resemble the across-the thickness variation of conventional axial plate waves. In the other case when the product trends to infinity, the Lamb wave modes degenerate into Raleigh waves, which will be confined to the plate surface.

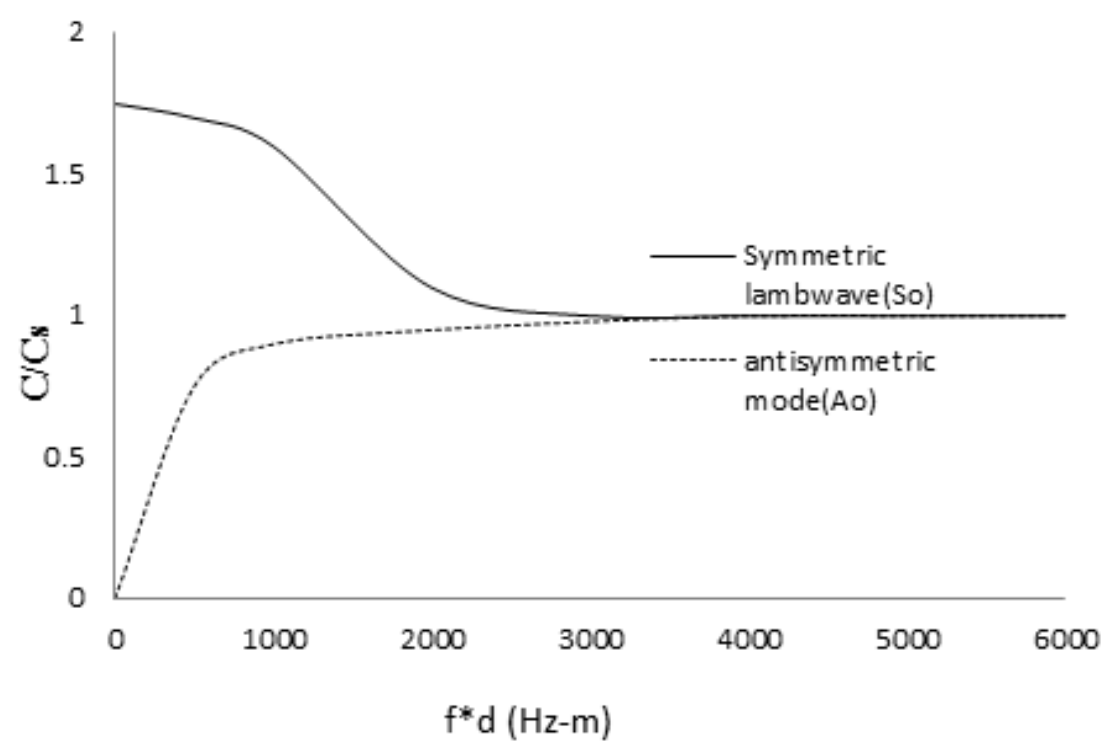

Figure 2.1 Comparison of Symmetric and Anti-Symmetric Lamb wave dispersion curves (lowest modes)

\subsection{Methods for Data Analysis in SHM}

The unprocessed Lamb wave perturbations could not predict the damage or wear effectively. Therefore, an effective signal processing technique is required to investigate the output data. The Fourier analysis gives the frequency response of the signal, which has been an effective tool during signal processing of stationary signals. The Fourier 
Transformation decomposes the signal into simpler pieces to analyze the signal (Pei and Yeh, 1998; Sejdiic et al., 2011). The Fast Fourier Transformation (FFT) was performs the the Fourier transformation more rapidly (Duhamel and Vetterli, 1990) But these techniques have a limitation when the signals characteristics vary with time. The Lamb

waves generated at the actuators in these experiments are non-stationary in nature and therefore Fourier analysis is not an appropriate signal processing tool to analyze the signals particularly to identify the localized variations in a processed signal.

\subsubsection{Wavelet Transformation}

A wavelet transform decomposes a time series into time- frequency-space domain and determines how the dominant modes vary with time. Wavelet analysis is similar to Fourier analysis in a way that it breaks a signal down into its constituents. It breaks the signal into its wavelets and scaled versions of mother wavelet. The translation and dilation operations performed to the mother wavelet describe the correlation between the wavelet and the localized portion of a signal. The scaling and translation processes during the wavelet decomposition are clearly showed by the trade ways company (Figure 2.2) during their elaborative description on wavelet analysis.

One dimensional (1-D) wavelet analysis was used to process a signal when the input data was a vector. The time series data taken on an oscilloscope could be analyzed using 1-D wavelet analysis. Two dimensional wavelets were used to compress and denoise the images and mainly used in image processing as described by Rout (2003) in his master's thesis titled: Orthogonal vs. Bi-orthogonal Wavelets for Image Compression). 
The data collected in the present research was one dimensional signal, and therefore 1-D analysis was performed in the following sections of the dissertation. From previous literature a perfect reconstruction orthogonal 1-D filter is presented below. If X $(\mathrm{Z})$ is the Z-transform of a the unprocessed signal $\mathrm{X}(\mathrm{t})$, a perfect reconstruction filter bank is constructed by an analysis and synthesis sub band passes as shown in the Figure 2.3 .

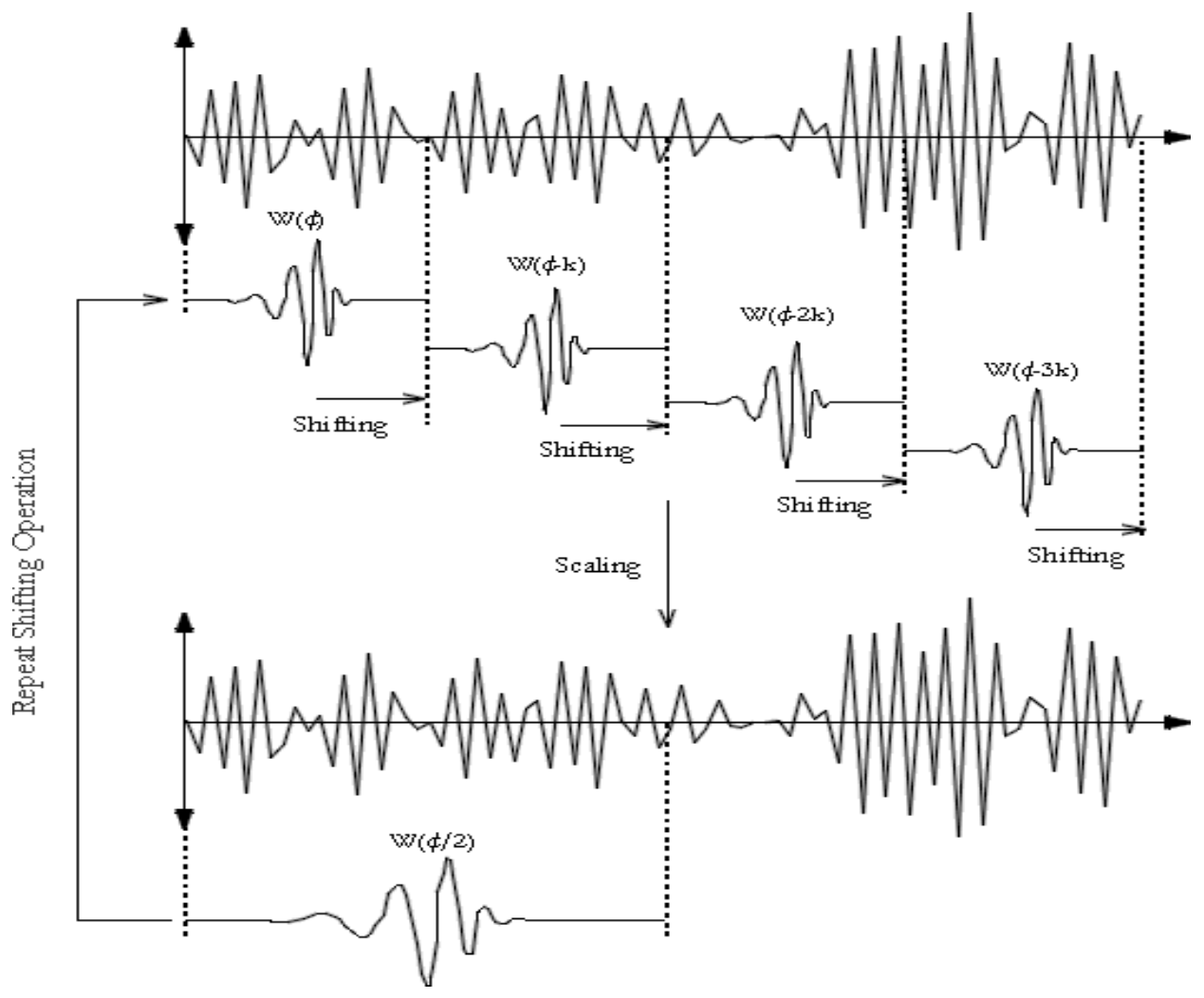

Figure 2.2 Shifting and Scaling operations during wavelet decomposition 


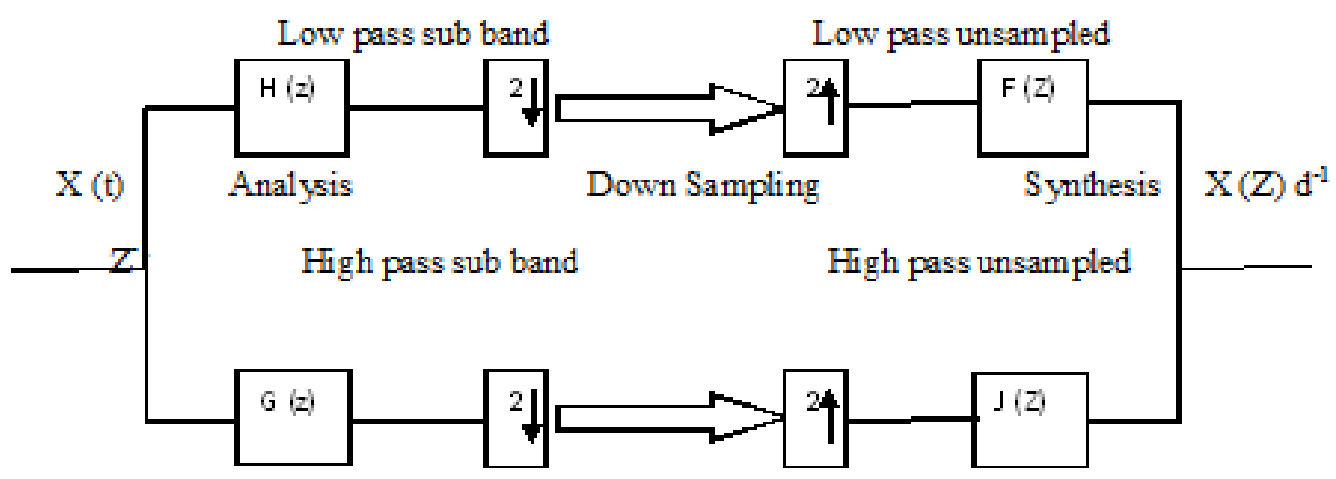

Figure 2.3 Perfectly reconstructed orthogonal filter bank

$\mathrm{H}(\mathrm{Z})$ and $\mathrm{G}(\mathrm{Z})$ is the low pass and high pass analysis filters. $F(Z)$ and $\mathrm{J}(\mathrm{Z})$ is the corresponding low pass and high pass filters to reconstruct the original signal with an overall delay $\mathrm{d}$.

The original signal of Continuous wavelet analysis can be reconstructed by integration over all the resulting frequency components (Lemistre and Balageas, 2001). The equation for the continuous wavelet transform of a signal data $\mathrm{X}(\mathrm{t})$ was obtained by convolving the data with mother wavelet $(\psi)$. The signal would be subjected to decomposition by the daughter wavelets; which are scaled $(\mathrm{s}>0)$ and translated $(\operatorname{tr} € R)$ interpretation of the original wavelet as shown in Figure 2.2 of this chapter. The equation for a Continuous Wavelet Transform (CWT) was derived by Vetteri and Herley (1992), and Strang and Nguyen (1996) in their famous books on Wavelets and Filter Banks, as cited in eq. (2.15). The original signal $\mathrm{X}(\mathrm{t})$ can be retrained back using an inverse wavelet transform as showed in eq. (2.16). 


$$
\begin{aligned}
& C W T(s, t r)=\frac{1}{\operatorname{sqrt}(s)} \int_{-\infty}^{+\infty} x(t) \psi\left(\frac{(t-t r)}{s}\right) d t \\
& X(t)=\frac{1}{s^{2}} \int_{-\infty}^{+\infty} \int_{0}^{\infty}\left[C W T(s, t r) \frac{1}{\operatorname{sqrt}(s)} \psi^{\prime}\left(\frac{(t-t r)}{s}\right)\right] d s d(t r)
\end{aligned}
$$

Since it was computationally very difficult to analyze signals using all wavelet coefficients, suggestion was made by the same others to pick up a discrete subset of the points to reconstruct a signal from corresponding wavelet coefficients. Analogically a Discrete Wavelet Transform (DWT) would look similar to a CWT while the convolution was between the signal data and a family of daughter wavelets as described in the following equation. In other words a DWT in its simplest form for a signal $X(t) € L^{2}$ is:

$$
D W T_{d, t r^{*}}=\int_{0}^{\infty} X(t) \psi_{d, t r^{*}}(\mathrm{t}) \mathrm{dt}
$$

The daughter wavelet family $\left(\psi_{d, t r}\right)$ was obtained by dilation of the mother wavelet. The suffix $d$ represents the dilation factor. Each response of the dilation factor represents a decomposition level of the wavelet. Unlike in CWT, $t r^{*}$ in a discrete wavelet transform represent dyadic translation. The original signal $X(t)$ can be reconstructed using an inverse discrete transform. The synthesis part of the signal was obtained by summation of the translated bands of all dilations (2.18).

$$
X(t)=\sum_{d}\left[\sum_{t r^{*}} D W T_{d, t r^{*}}\right] \psi_{d, t r^{*}}(\mathrm{t})
$$

This process of analyzing $\mathrm{X}(\mathrm{t})$ by decomposing into multi levels of different band widths, and again synthesizing to obtain the discrete wavelet is called multi resolution. Previously researchers (Mallat, 1989; Kim et al., 2006) developed such wavelet families for signal and Image processing. 


\subsubsection{The S-Transformation}

Stockwell et al. (1996) developed the s-transformation to improve the time and frequency resolution of the short time Fourier Transformation (STFT):

$$
S(\tau, f)=\int_{-\infty}^{\infty} x(t) \frac{|f|}{\sqrt{2 \pi}} \exp \left[\frac{-f^{2}(\tau-t)^{2}}{2}\right] \exp (-i 2 \pi f t) d t
$$

$\mathrm{x}(\mathrm{t})$, is the signal in the time domain. $t$ and $f$ are the time and frequency respectively. The inverse transform of the s-transformation may be calculated with the following equation:

$$
x(t)=\int_{-\infty}^{\infty}\left[\int_{-\infty}^{\infty} S(\tau, f) d \tau\right] \exp (i 2 \pi f t) d f
$$

The s-transformation envelopes of the Lamb wave signals will be plotted using eq. (2.19) and eq. (2.20) in Chapter Six.

\subsection{Monitoring Tool Wear in Machining}

Manufacturing cost comprises a major portion of the total cost of a product, and it could be reduced by increasing the machine utilization time. Frequently serious interruption in the manufacturing process occurs due the catastrophic failure of cutting tools. Automatic tool wear monitoring is very important in present day manufacturing to monitor the tool condition and decrease the production cost. Tool wear monitoring identifies the actual state of tool wear, and evaluates the remaining life of the tool. The dimensions of the components manufactured under tool wear conditions could result in 
mismatch during assembly. Tool wear monitoring also prevents machine tools from sudden failures.

Previously force sensors, torque sensors, accelerometers and acoustic emission probes were developed to monitor tool wear. Drill operation generates blurs due to plastic deformation. These blurs cause dimensional errors and misalignments during assembly. In order to avert the extra effort and time for deburr operations, an automated tool wear monitoring method that adopt spindle torque signals was demonstrated by Pena et al. (2005). This method detects the low height burr information that would not affect the dimensions of the machined components. Huseyin et al. (2004) developed a method to identify tool wear status based on cutting force and torque measurements using hidden markov models. The vibration characteristics of a machining process determined the state of tool wear. Metin and Oysu (2004) found that the damping ratio measured by impact excitation would enhance the tool wear development, and therefore the tool wear status was successfully estimated using the damping ratio measured by impact excitation.

An acoustic wave is a stress wave generated by sudden release of energy in elastic materials. Acoustic emission based methodologies were also successful to monitor tool wear (Li, 2002; Kivio et al., 2003). Acoustic emission signals were analyzed to describe the breakage monitoring in a multi spindle drilling machine. The increase in spectral power in low and high frequency bands predict the breakage of the tool.

An Online toll wear monitoring method was described by Castejon et al. (2007) and a tool wear probe was designed by Szararczyk et al. (2004) for Numerical Control (NC) lathes. This method classified the tool wear into three levels called low, medium 
and high, i.e., the technique automatically recognized the condition of the tool and allowed to replace the tool after medium level of wear, and the probe was able to monitor the damage successfully at the tip of the cutting edge on NC lathe.

Previously, optimization of the life time of a tool ensuring the dimensional tolerance of a product was demonstrated using a statistical signal processing methodology (Prickett and Johns, 1999; Stoic and Raos, 2003; Verl et al., 2009). Multiple sensor systems were used to increase the intelligence level of machine tool condition monitoring of a milling process, and s ensor less automated condition monitoring was performed to compute the characteristic parameters that enable tool wear.

The wear monitoring in Mill tool was studied earlier using cutting force models (Altinta et al, 1988) and various signal processing tools like wavelet transforms and neural networks (Tansel et al, 1995). The work proposes a new methodology that adopts guided waves for effective mill tool wear monitoring. Piezoelectric elements were already proved to be efficient to detect damages and for health monitoring in aluminum plates (Lee and Stazewski, 2003, 2007; Rajic et al.). A combination of piezoelectric sensors, Lamb waves and signal processing techniques were effectively established in this dissertation work to effectively monitor the wear of an end mill tool.

Teti et. al., (2010) reviewed the past contributions of different machining processes and the condition monitoring techniques used in SHM. The sensor systems and the signal processing methods that were used to monitor various imperfections and features of a cutting tool are listed in Table 2.1. Byrne et.al. (1995) in their keynote paper described various sensor methods and signal processing techniques used during various 
cutting operations. A brief summary of the sensor research for tool and process monitoring in machining is showed in Table 2.2

Table 2.2: Summary of tool condition monitoring features

\begin{tabular}{|l|l|l|l|}
\hline Monitoring Feature & Sensor System & Signal Analysis & Machining \\
& & & Process \\
\hline Tool Wear & Acoustic emission & Wavelet transform & Milling \\
Tool Geometry & Vibrations & $\begin{array}{l}\text { Time and frequency } \\
\text { domain analysis } \\
\text { Tool Temperature }\end{array}$ & Band sawing \\
& Cutting force & Bydraulic pressure & Broaching \\
& Motor Power & Histogram method & Drilling \\
& Laser & Thermal analysis & Turning \\
& Camera & Fractal dimensions & \\
& Thermal Imaging & & \\
& Audible Sound & & \\
& & & \\
& & & \\
\end{tabular}


Table2.3: Sensor systems and signal processing techniques used in various cutting processes

\begin{tabular}{|l|l|l|}
\hline Cutting Process & Sensor System & Signal Processing Technique \\
\hline Turning & AE & FFT \\
Milling & Force & NN \\
Drinding & Vibration & Average power \\
Multing spindle drilling & Temperature & RMS \\
& Power & Auto Regressive moving average \\
& Vision & GA \\
& Acceleration probe & \\
\hline
\end{tabular}

\subsection{Brief Summary}

This chapter performed a detailed survey of the previous research in the field of SHM. Piezoelectric materials and their application during SHM were demonstrated in Section 2.2. The previous studies of Electromechanical Impedance method and guided surface waves used in SHM were presented. Important signal processing techniques that supported during the analysis part of the present research were reviewed in the Section 2.4. Previous tool monitoring techniques in machining were studied in Section 2.5. The dissertation work in the following chapters inspected the complex geometry parts and welds for imperfections based on the previous literature studied in this chapter. 


\section{CHAPTER 3}

\section{EXPERIMENTAL SETUP}

One of the main contributions of this dissertation work is to estimate the electrical performance of the piezoelectric material actuators under induced mechanical stress. This chapter describes the experimental procedures adopted to estimate the performance as well to determine the reliability of piezoelectric materials as inspection actuators. A detailed experimental procedure of investigating the electrical performance of the piezoelectric beams is demonstrated. It also specifies the experimental setup to evaluate the utility performance of piezoelectric elements as sensors and receiving resonators to monitor wear in an end mill.

\subsection{Experimental Setup to Investigate Electrical Response}

Piezoelectric materials have a crystalline structure that provides them with the ability to transform mechanical strain energy into electrical charge and, vice versa, to convert an applied electrical potential into mechanical strain. This property provides these materials with the ability to absorb mechanical energy from their surroundings, usually ambient vibration, and transform it into electrical energy that can be used to power other devices. Conversely With an electrical excitation they can be used to determine mechanical flaws with in a structure.

Actuators using piezoelectric materials have been developed by manufacturers like American Piezo Corporation (APC) International Limited. Bi morph piezo electric 
actuators and the disc resonators were purchased from APC Corporation to conduct various experiments in the laboratory.

This section describes the experimental procedure to find the natural frequency and the nonlinear performance of the piezoelectric bimorph actuators. The natural frequency of the piezoelectric elements manufactured my APC International Limited (catalog no. 40-1010) was determined and the voltage output estimated using a two small (12 $\mathrm{mm}$ long $8 \mathrm{~mm}$ wide and $0.6 \mathrm{~mm}$ thick) piezoelectric Actuators.

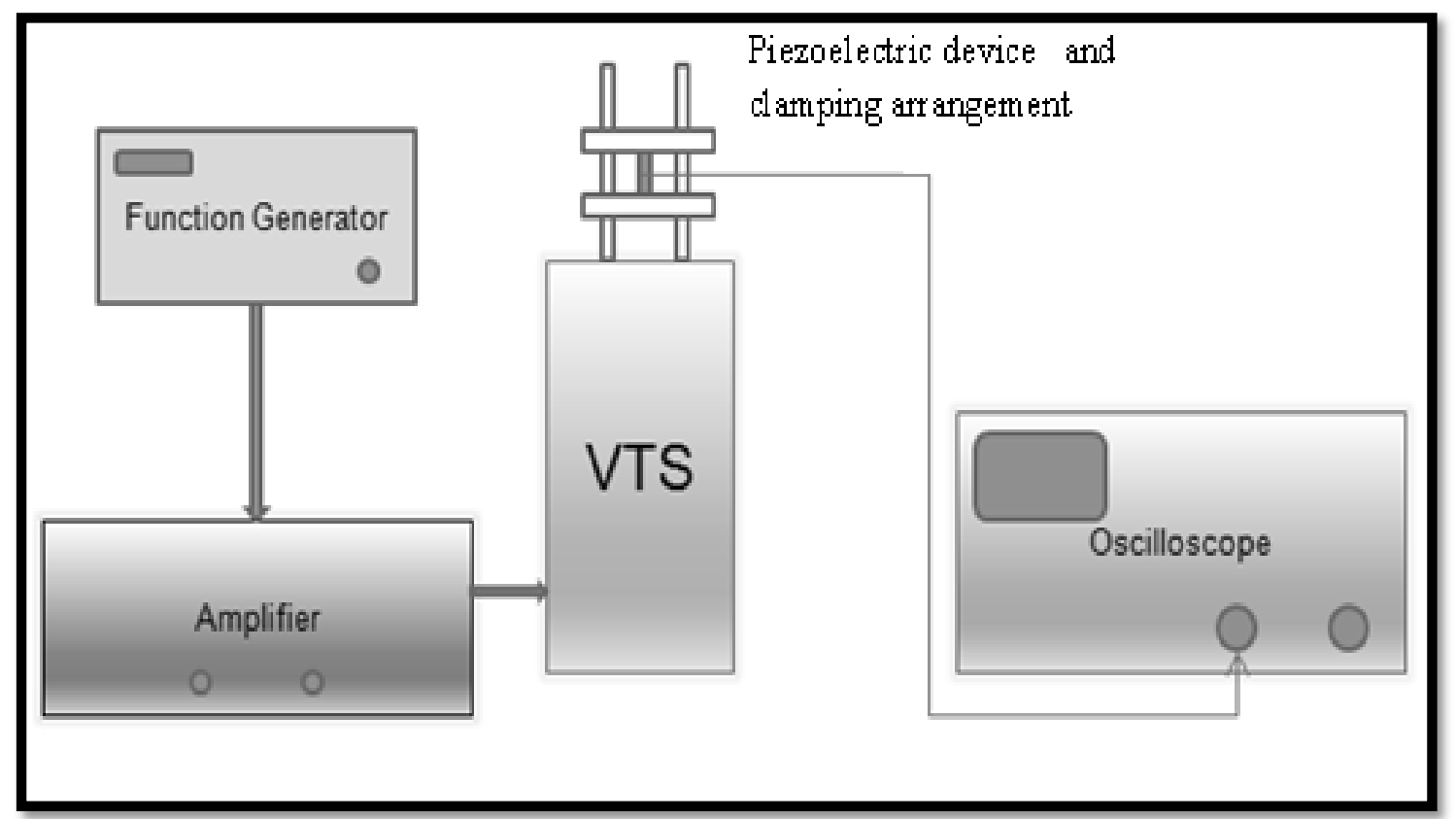

Figure 3.1 Schematic diagrams representing the experimental setup that evaluated frequency response 
The experimental setup consists of a BK Precision 4017 signal generator, a Macro Tech Crown XTI 1000 power amplifier, and a vibration test system. The frequency response was recorded on a using a Stanford Research Systems model SR780 two channel network analyzer. The impedance of the data acquisition system was $1 \mathrm{M} \Omega$. The schematic and the experimental setup that describes the experimental procedure are showed in Figure 3.1 and Figure 3.2.

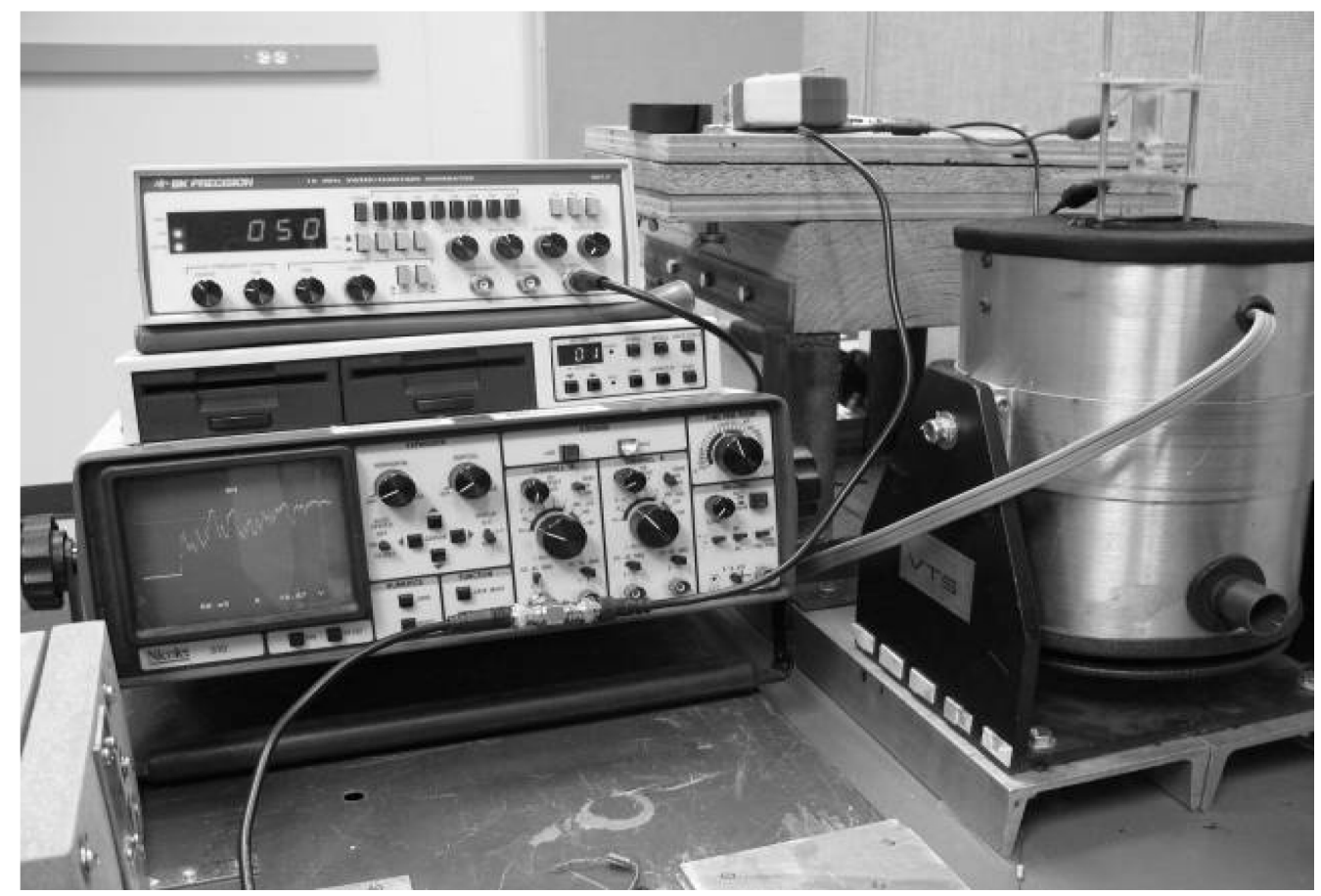

Figure 3.2 Experimental setup used to investigate the electrical response and nonlinear characteristics of piezoelectric actuators

The specimens for the experiment to test the electrical performance were prepared from the small piezoelectric elements $(12 \mathrm{~mm}$ long $8 \mathrm{~mm}$ wide and $0.6 \mathrm{~mm}$ thick) purchased from APC International Corporation. Two different shapes (Round and 
Rectangular) were designed from $0.2 \mathrm{~mm}$ thick aluminum case. The two newly formed bimorphs were fixed at an optimum distance in a $4.8 \mathrm{~cm} \mathrm{X} 1.5 \mathrm{~cm} \mathrm{X} 1.5 \mathrm{~cm}$ dimensional aluminum tube as showed in Figure 3.3.

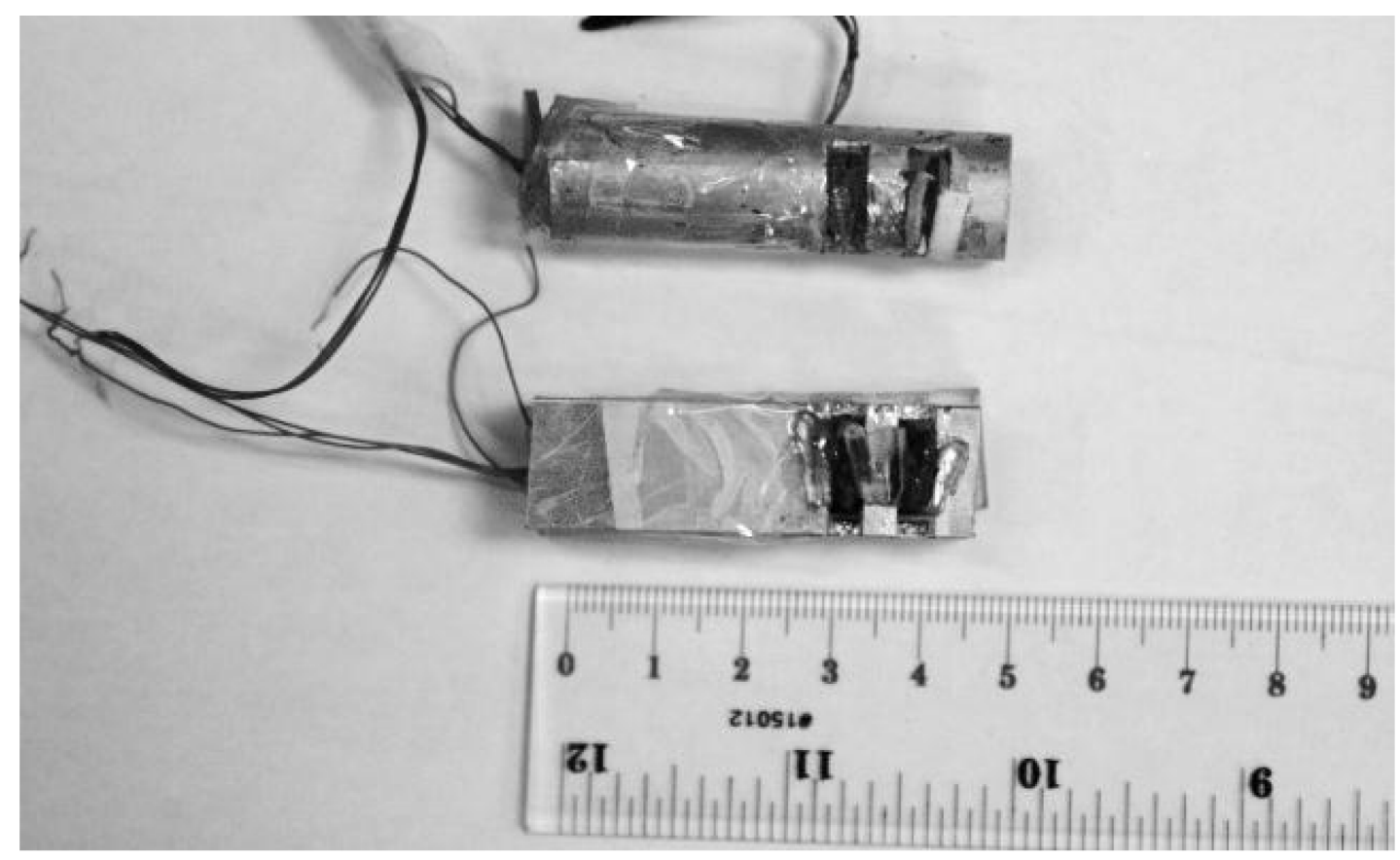

Figure 3.3 Comparison of the electrical response testing devices (Round, and Rectangular shapes)

A rectifying circuit consisting of a $25 \mathrm{~V}(10 \mu \mathrm{F})$ capacitor along with four diodes was connected to the piezoelectric actuators and installed inside the aluminum case, for both round and rectangular designs in order to convert the generated AC current into DC. The DC voltage generated would be useful to charge the rechargeable batteries. The rectifying circuit design is presented in Figure 3.4 (a) and the simulation of the output of the circuit is presented in Figure 3.4 (b). 


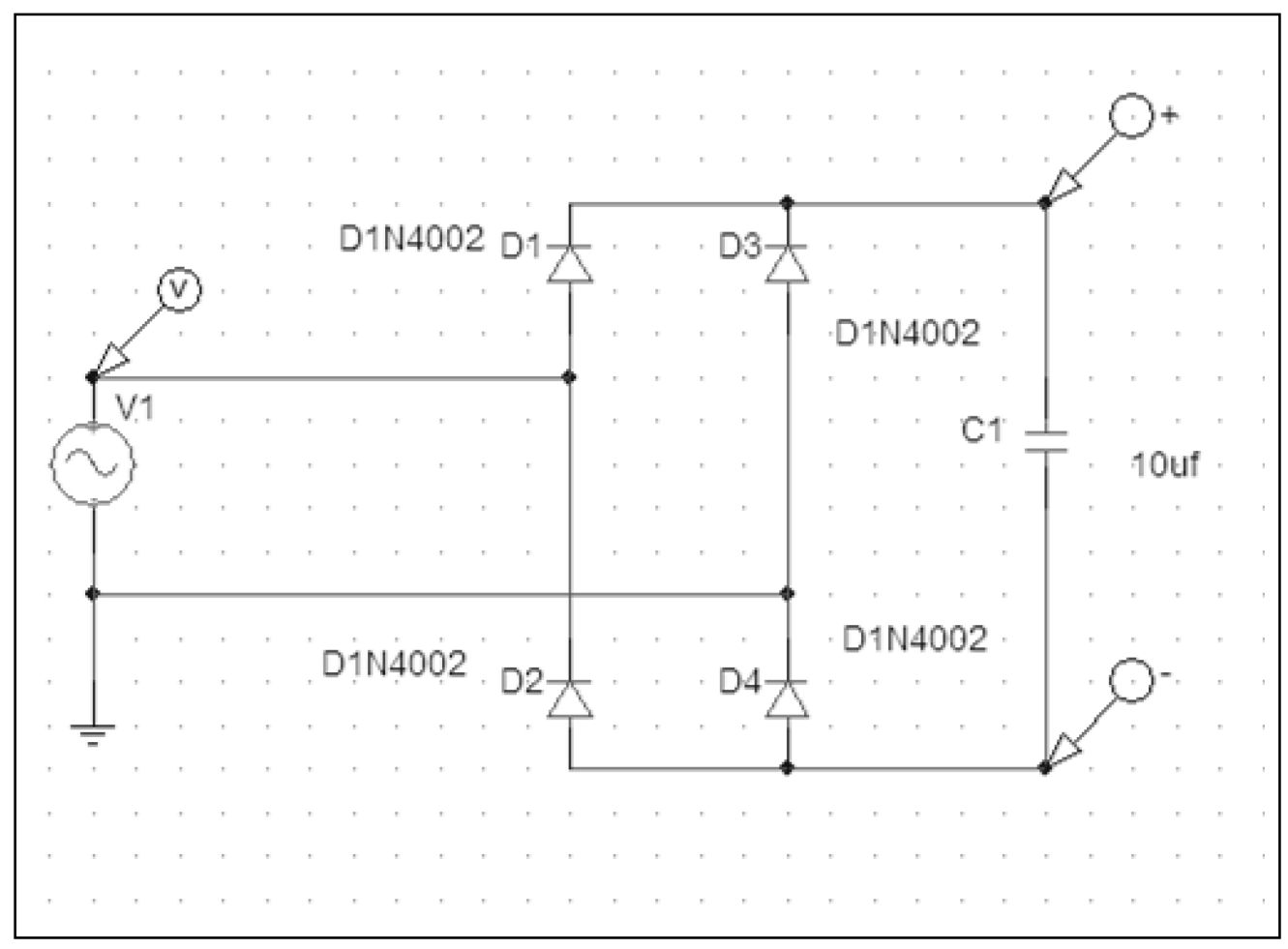

(a)

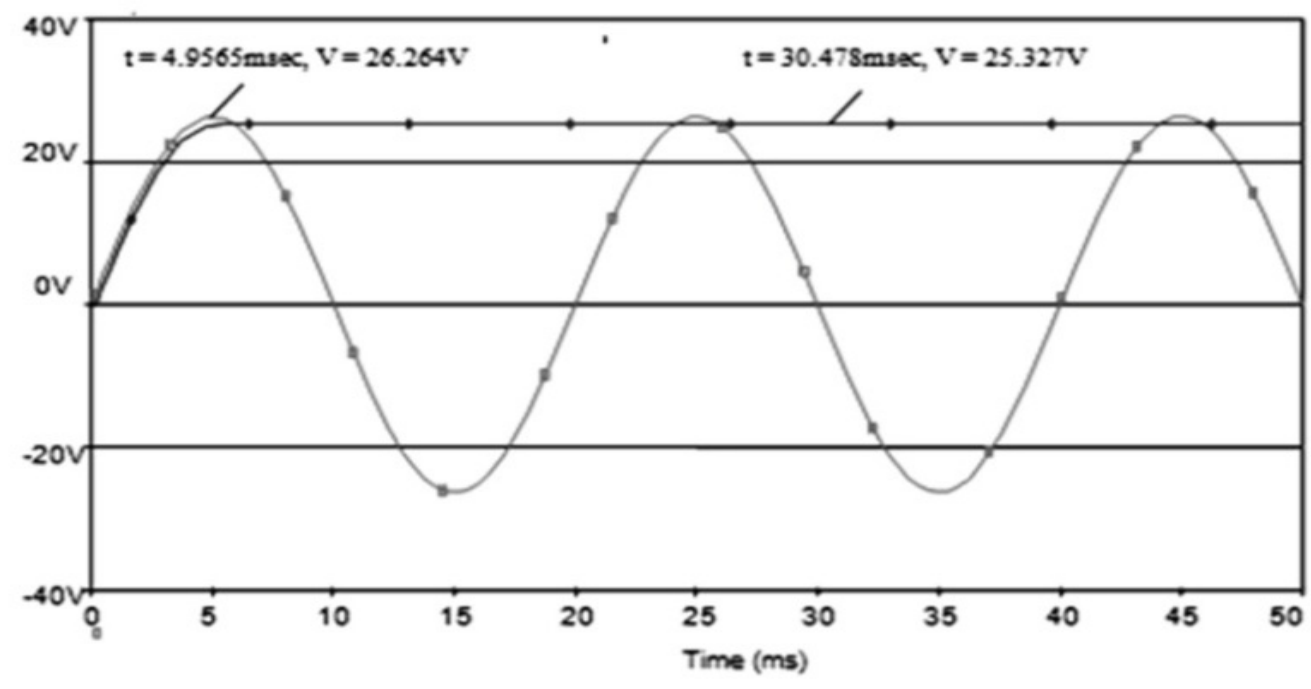

(b)

Figure 3.4 Circuit diagram and simulation to convert AC output to DC: (a) Rectifying circuit (b) Simulation of the circuit to determine DC output voltage 
The designed aluminum cases with the actuator and electrical circuit attachments are then placed between two wooden fixtures on a vibration test system. A similar experimental set up showed in Figure 3.2 was used to analyze the performance of the bimorphs. Detailed analysis of results of the experiments described in section 3.1 will be explicated in Chapter Four.

\subsection{Experimental Setup to Inspect Complex Geometry Parts and Welds}

Another important contribution to this research work is to discover the capabilities of piezoelectric materials to sense and receive surface waves and response of signals with complex geometry cutting tool (End Mill). The evaluation of degradation performance of piezoelectric disc sensors was performed using surface waves. Piezoelectric disc materials were characterized under mechanical stress conditions and when they were subjected to tensile, compressive and fatigue loads during earlier experiments conducted at out laboratory. Lamb waves were used as propagating waves

during the experiments. In the following sections the applicability and effectiveness of piezoelectric disc actuators for damage detection during structural health monitoring is established.

\subsubsection{Monitoring Wear of an End Mill}

The wear generated on the mill tool during the experiment was analyzed simultaneously by establishing two different methodologies. The first method used the surface elastic waves called Lamb waves as the sensing, transmitted and receiver signals. 
The second method uses a surface response to a chirp signal excitation to determine the level of damage in an end mill tool.

Initially a picket 2 circuit is prepared on a bread board with a microchip (pic16F690) was used to generate the Lamb waves. The quality of the Lamb waves are observed on a Tektronix (TDS 2024B) four channel oscilloscope. These set up preliminarily serve the purpose of generating the Lamb waves, but it could not be able to generate accurate surface waves that could efficiently analyze the data for detection of the damage. A Métis Design SHM Node MD 75602 setup was established to generate accurate Lamb waves as shown in Figure 3.6. The generated Lamb waves were monitored on a Tektronix oscilloscope to check the characteristics of the signal.

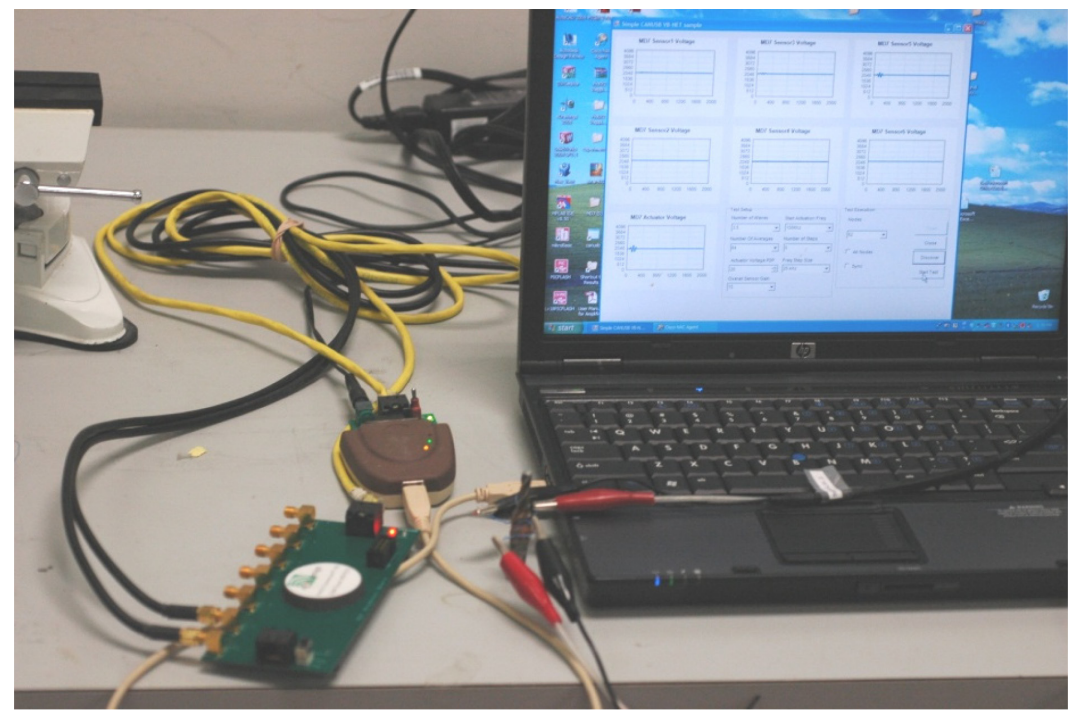

Figure 3.5 Experimental setup that classified end mill tool wear using Lamb wave method (Métis Design SHM Node MD 7502) 
The experimental setup to record the surface response due to the excitation at one of the actuators is showed in Figure 3.8. A Stanford Research Systems (SRS) Model SR780 signal analyzer was used for the experiment. The Two channels of this signal analyzer are utilized as both a signal generator and an oscilloscope to record the signal. The Analyzer can generate a chirp signal excitation at one of two piezoelectric elements attached to the mill tool. The surface response to excitation was recorded at channel 2 of the signal analyzer during 2000 data points. The time domain signals and the frequencyamplitude plots at various stages of wear were recorded and showed in Figure 3.9. Data1 represents the no wear condition of the mill tool. Data2-Data6 denotes the five different (Low, Medium and Severe) stage of the tool wear.

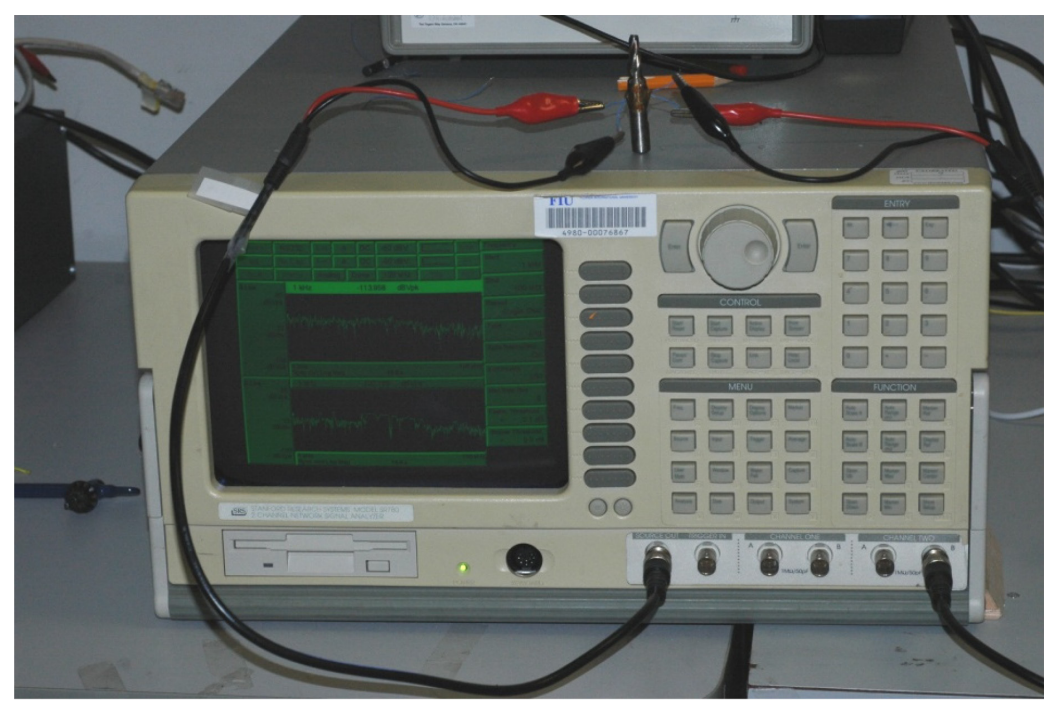

Figure 3.6 Experimental setup used to record the surface response to excitation signals

Piezoelectric disc actuators were purchased from APC International $(0.25$ inch diameter) to install on the mill tool as signal sensors and receivers. M Bond 200 glue was 
used to attach them on the mill tool of Length 2.5 inch and diameter 0.25 inch. Two disc actuators were installed on either of the sides on the shank portion of the end mill. One of the two discs acted as a resonator and the other was used to receive the propagated signal. An artificial wear was created at the tip of the flank portion of the tool in five stages using a grinding machine.

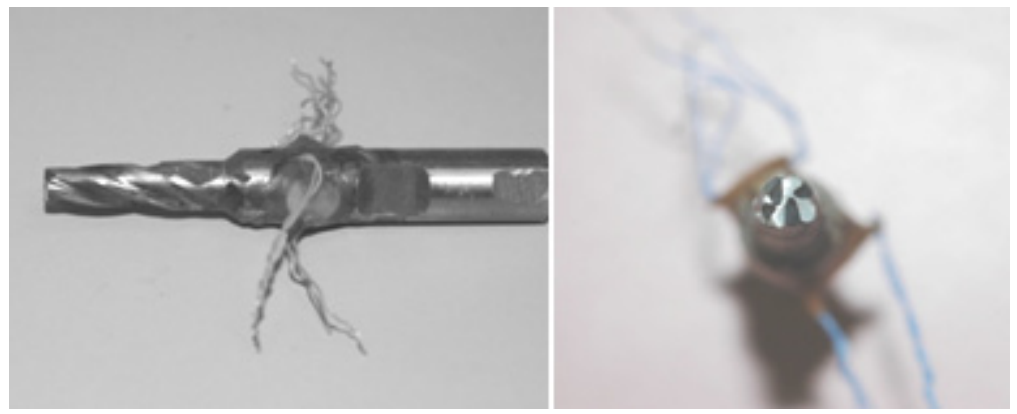

(a) End mill tool

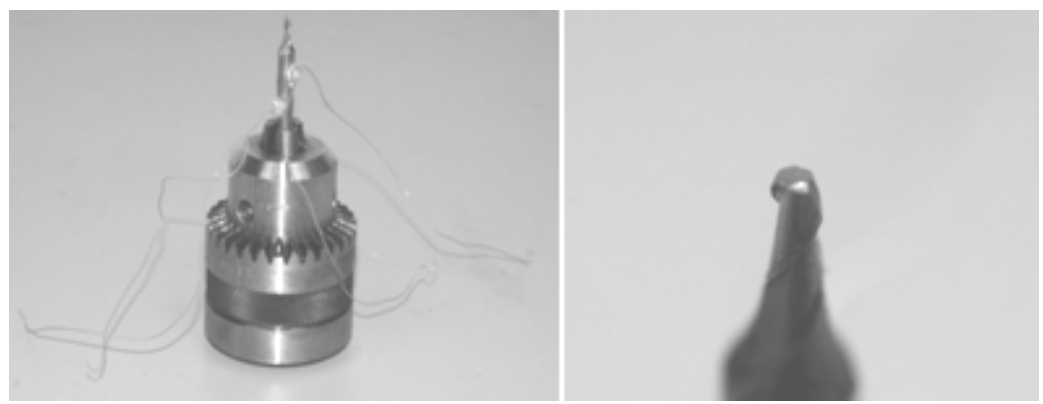

(b) Micro mill tool

Figure 3.7 Sensor attachments and wear on the end mill specimens

The same experiment was repeated with a micro mill tool. The tools and different stages of wear for the mill tool and micro mill tool are showed in figure 3.7(a) and 3.7 
(b). The first step indicated a low wear, second and third stages denoted a medium wear, and in the last two stages a sever wear was induced on the tool.

\subsubsection{Detecting Imperfections in Welds}

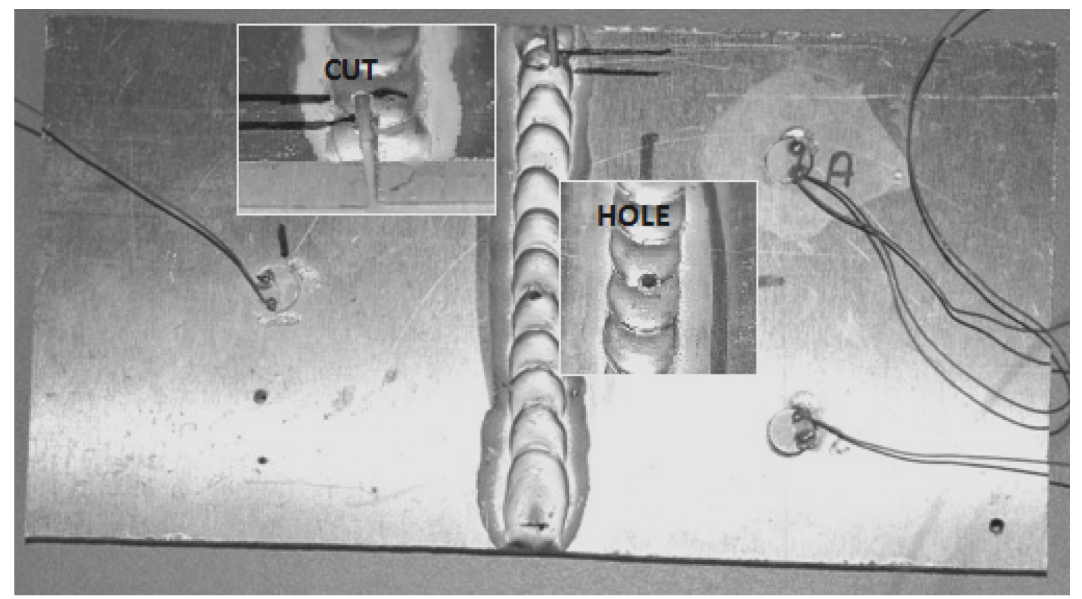

Figure 3.8 Butt weld joint with a $1 / 4^{\text {th }}$ inch cut and $1 \mathrm{~mm}$ diameter hole

A similar experimental setup as described in section 3.2 was established to analyze the flaws occur on welds. A butt weld joint is prepared between two 3 inch length, 3 inch wide and 0.25 inch thickness aluminum plates as showed in Figure 3.10. Two piezoelectric actuators were installed on the aluminum plates at either sides of the weld. The experiment was conducted using both Lamb wave perturbations and the surface response to excitation methods.

\subsection{Brief Summary of the Chapter}

Chapter Three demonstrated the experimental procedures during the investigation of electric response and damage detection characteristics of piezoelectric actuators. Experimental setups to investigate the electrical output of compact energy harvesting 
devices were discussed in Section 3.1. Experiment setup for evaluation of imperfections in complex structures, and the data acquisition methodologies were elaborately discussed in the chapter. The data obtained in the chapter will be analyzed in Chapters Five and Six. 


\section{CHAPTER 4}

\section{PROPOSED PROCEDURES FOR DATA ANALYSIS}

\subsection{Lamb Wave Procedure}

. Ultrasonic waves in flat plates were first described by Lamb (1917) and a broad analysis was given by Royer and Dieulesaint (2000). Lamb waves are guided ultrasonic waves and travel inside the plates with vertical polarization. Lamb waves appear in the two modes called symmetric and anti-symmetric. These waves were simulated at 680 $\mathrm{kHz}$ frequency using a 2D Lamb wave simulation software, and the dispersive nature was compared in Figure 4.1 (a) and Figure 4.1(b).

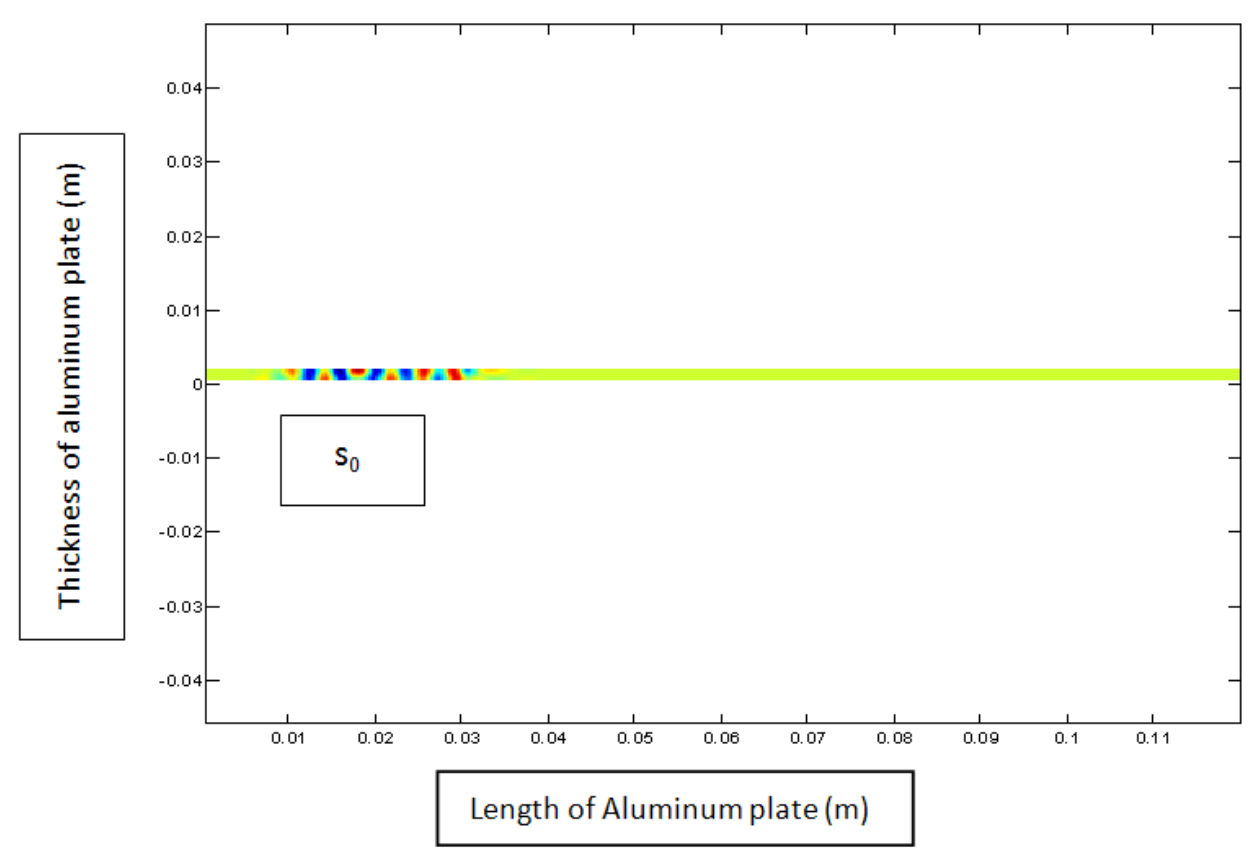

(a)Symmetric mode $\left(\mathrm{S}_{0}\right)$ 


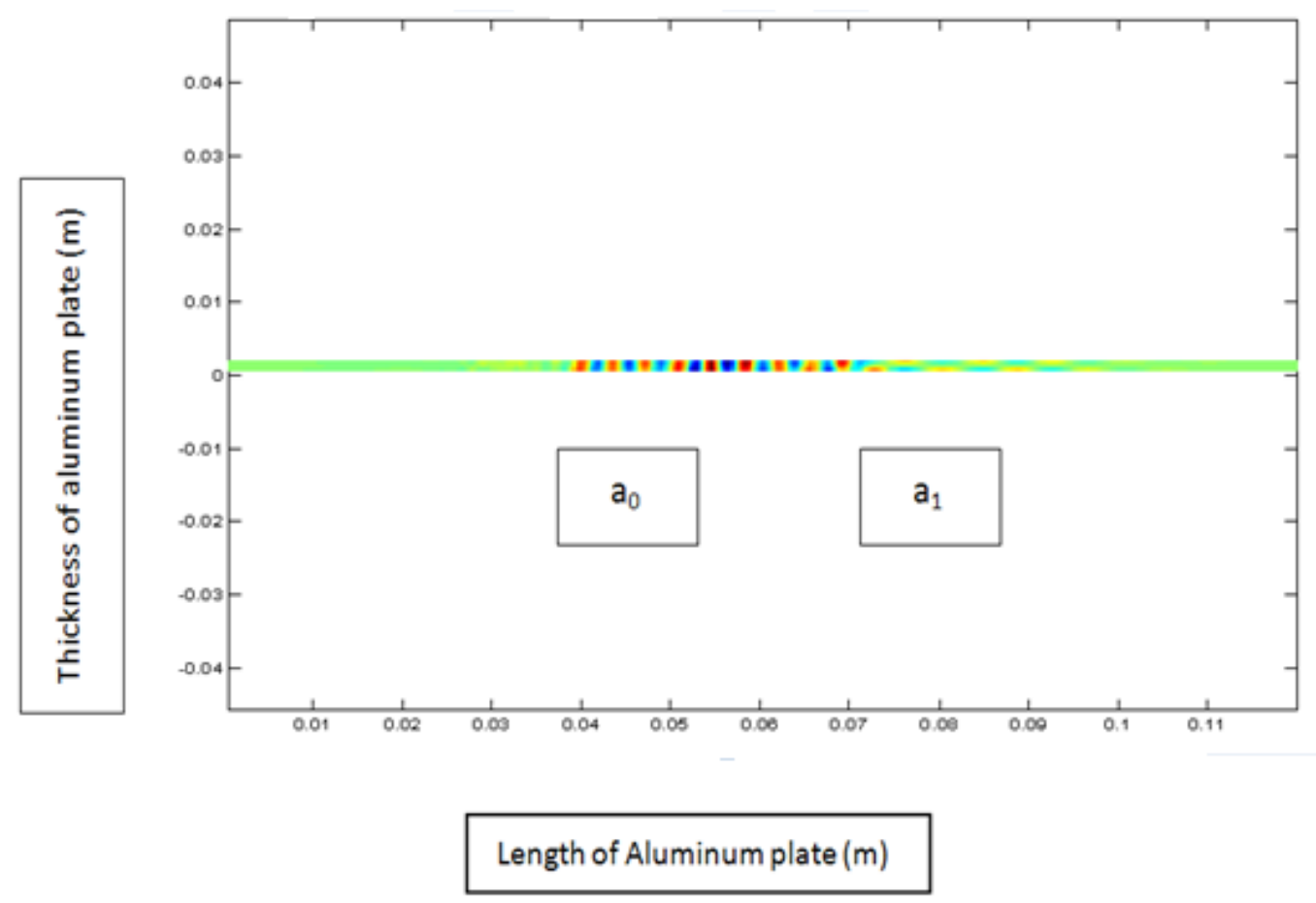

(b) Anti-Symmetric mode $\left(\mathrm{A}_{0}\right)$

Figure 4.1 Simulation of Lamb wave modes (Excitation frequency: $680 \mathrm{kHz}$ )

Symmetric mode Lamb waves are longitudinal and travel at same speed, while anti-symmetric mode Lamb waves called flexural waves are much dispersive in nature. The schematic that described operating principle to record the Lamb wave data is showed in Figure 4.2. A Metis design SHM node MD 7052 chip consists of an actuator and six sensor ports. Two piezoelectric elements were connected on both the sides of the milling tool. The metis design was used to actuate one of the piezoelectric elements attached on the tool surface. The piezoelectric element attached on the other surface sensed the transmitted Lamb wave signals. 
An interface was used between the metis design SHM MD 7052 and a computer to record the data on the computer. Progressive wear was generated on the cutting tool in five steps, and the Lamb wave data due to the wear was collected.

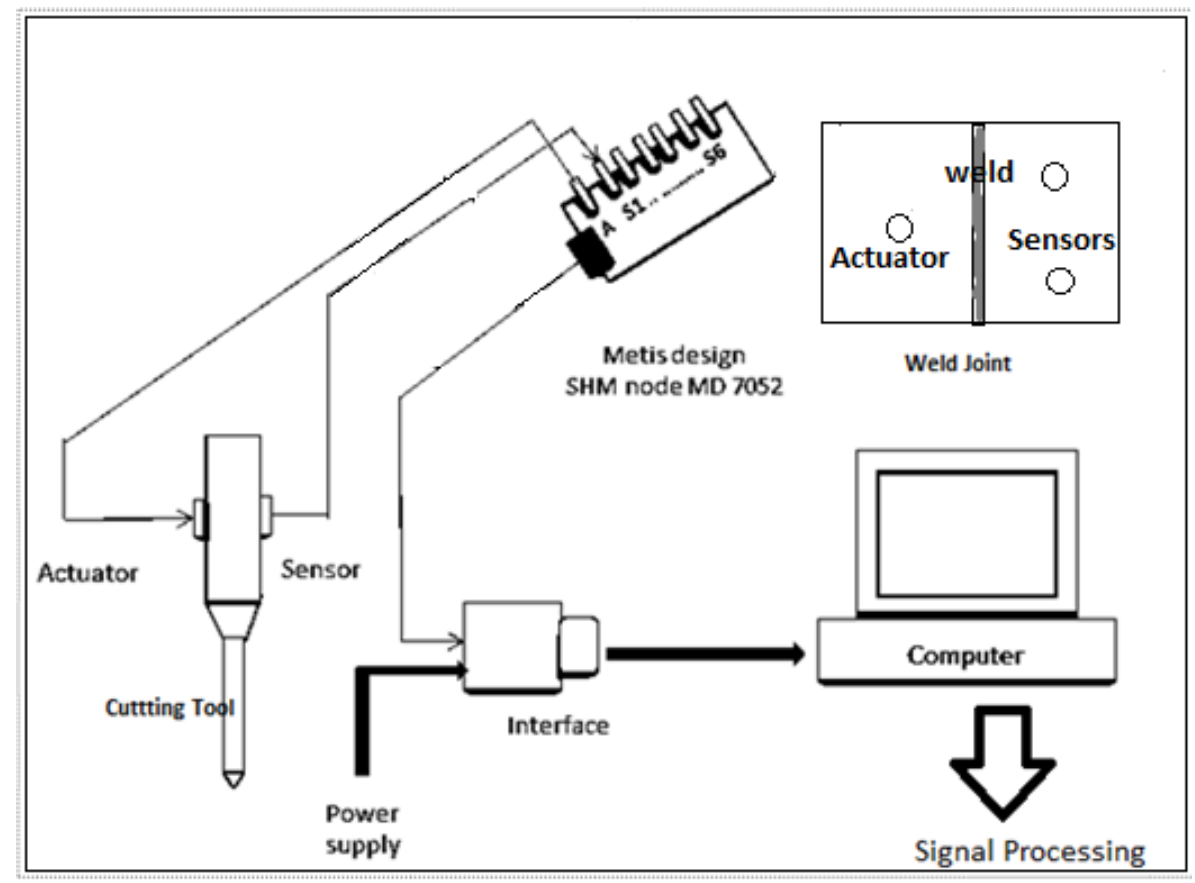

Figure 4.2 Data analysis using Lamb wave technique

The same principle was used to record the Lamb wave data on a weld joint. The actuator/sensor setup of the weld joint is showed in the Figure 4.2. The actuator node of the metis design excited the actuating piezoelectric element on the left side of the weld. Two piezoelectric elements were placed on the right side of the weld, which sensed the Lamb wave perturbations that pass through the weld medium. The actuator and sensor data was recorded on the computer.

The data collected using the Lamb wave method analysis was performed in Chapter Five using the s-transformation. The theoretical background of the s- 
transformation was described in Section 2.3.2. s-transformation and their envelopes were used to estimate the data obtained during the Lamb wave method.

\subsection{Surface Response to Excitation (SuRE) Technique}

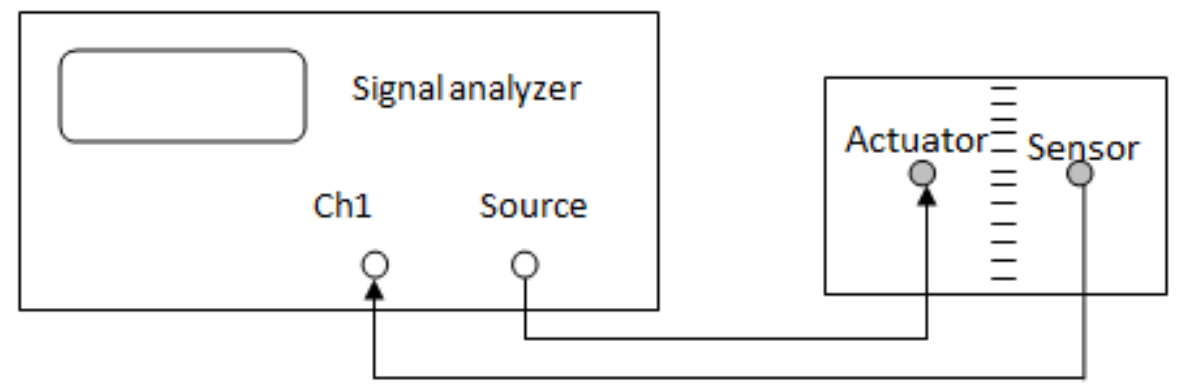

Figure 4.3 SuRE Technique to detect damages on a weld joint.

The SuRE principle that collects the surface response data on a weld joint is described in Figure 4.3. The end mill described in the Lamb wave approach was also tested using the SuRE method. In SuRE, A sweep sine wave was generated on a signal analyzer, which is used to determine the imperfections of the end mill and a weld joint. The sweep sine wave excited at one of the piezoelectric elements that marked as actuator. The surface response data due to the excitation was collected using the other piezoelectric element that was attached on the other side of the joint. The sweep sine wave data obtained during the experiment was analyzed to determine the wear and damage characteristics.

Sum of Square of the difference values were determined from the SuRE data to analyze the damages in cutting tools and welds. The square of difference $\left(x_{i+1}^{2}-x_{i}^{2}\right)$ was 
calculated between two successive data points $x_{i}$ and $x_{i+1}$. This analysis was performed during all stage of the wear, and during dissimilar frequency intervals. The variation in significant peaks in data was compared to determine the wear level of the tool using SuRE approach. The complete discussion of the results will be presented in Chapter Six.

\subsection{Bi-orthogonal Wavelet (Binlet) Analysis}

Wavelet transforms decompose time series signals into frequency as well as space simultaneously. The signal processing of the experimental results was performed by using bi-orthogonal filters in MATLAB (wavelet tool box) environment. The capability of bi-orthogonal synthesis and analysis filters to recognize various kinds of damages and wear in welds and cutting tools is demonstrated in the following paragraphs.

The multi resolution wavelets, where the synthesis filteres are the transposes of analysis filters are named as orhogonal wavelets (Strang and Nguyen 1996 ). The scaling functions in biorthogonal filters for analyis were denoted by the dilation entity $\tilde{\phi}(t)$ and tranlation as $\tilde{\phi}\left(t-t r^{*}\right)$ in a particular multireolution space. The wavelets of scaling and translation can be expressed as $(\widetilde{w}(t))$ and $\widetilde{w}\left(t-t r^{*}\right)$ in the corresponding multi resolutin space . The bi-orthogonoal anlysis dilation and wavelet values can be obtained using the following equations (4.1 and 4.2).

$$
\begin{aligned}
& \widetilde{w}(t)=\sum_{\mathrm{tr}^{*}=0}^{\mathrm{N}} 2 \mathrm{~h}_{1}\left(t r^{*}\right) \widetilde{\phi}\left(2 t-t r^{*}\right) \\
& \widetilde{\phi}(t)=\sum_{\mathrm{tr}^{*}=0}^{\widetilde{\mathrm{N}}} 2 \mathrm{~h}_{0}\left(t r^{*}\right) \widetilde{\phi}\left(2 t-t r^{*}\right)
\end{aligned}
$$


The dilatiion and tranlation of functions mention above represents at each level of multiresolution (Ali and Haddad, 2001). The suceeding scale and wavelet values of a multiresolution space can be obtained by adding scale function to the of the corresponding wavelet :

$$
\begin{gathered}
\tilde{V}_{j+1}=\tilde{V}_{j}+\widetilde{W}_{j} . \\
\tilde{V}_{j}=\text { scale of of } j^{\text {th }} \text { multi resolution space } \\
\widetilde{W}_{j}=\text { corresponding wavelet }
\end{gathered}
$$

The high pass analysis multi resolution space coefficients $\left(\mathrm{H}_{0}=\left\{h_{0}, h_{1}, h_{2}, \ldots\right\}\right)$ in bi-orthogonal filters differs from orthogonal filters. The high pass analysis bi-orthogonal filter coefficients are not orthogonal to each other instead they are bi-orthogonal to their corresponding synthesis filter coefficients $\left(\mathrm{F}_{0}=\left\{f_{0}, f_{1}, f_{2}, \ldots\right\}\right)$. The analysis wavelet of $\mathrm{j}$ th multi resolution space would be bi-orthogonal to its corresponding synthesis dilate and vice versa. Also in continuous time the synthesis dilation function $\phi(t-$ tr) and synthesis wavelet function $w\left(t-t r^{\prime}\right)$ are bi-orthogonal to corresponding analysis functions even when $\left(t r \neq t r^{\prime}\right)$. The transform and inverse bi-orthogonal wavelet transform were described by Strang and Nguyen, 1996 as cited in eq. (4.4) and eq. (4.5).

$$
\begin{aligned}
& X(t)=\sum_{-\infty}^{\infty} \sum_{-\infty}^{\infty} \tilde{b}_{j k} w_{j k}(t) d t \\
& \tilde{b}_{j k}=\int_{-\infty}^{\infty} X(t) \widetilde{w}_{j k}(t) d t
\end{aligned}
$$

$w_{j k}(t)$, represents the dyadic synthesis wavelet 


$$
\begin{aligned}
& \widetilde{w}_{j k}(t), \quad \text { represents the analysis dyadic wavelet } \\
& \tilde{b}_{j k}, \quad \text { represents the coefficients during analysis }
\end{aligned}
$$

A binary coefficient or dyadic coefficient (Abdukiram et al., 2005) is an integer divided by a power of 2 . The "binlet" family consists of various high pass and low passes dyadic coefficients of bi-orthogonal decomposition and reconstruction filter banks. The Wavelet tool box window of MATLAB provided various combinations high pass and low pass dyadic coefficient of bi-orthogonal filters to analyze the data. The maximum number of high and low pass dyadic coefficients that were used in the wavelet tool box window was confined to eight. Strang et al., 1996 derived bi-orthogonal filter with more low and high pass coefficients. The low and high pass decomposition filters (DF) and reconstruction filters $(\mathrm{RF})$ were described in Figure 4.4. The number of coefficients was denoted by $\mathrm{Nd}$ and $\mathrm{Nr}$ respectively.

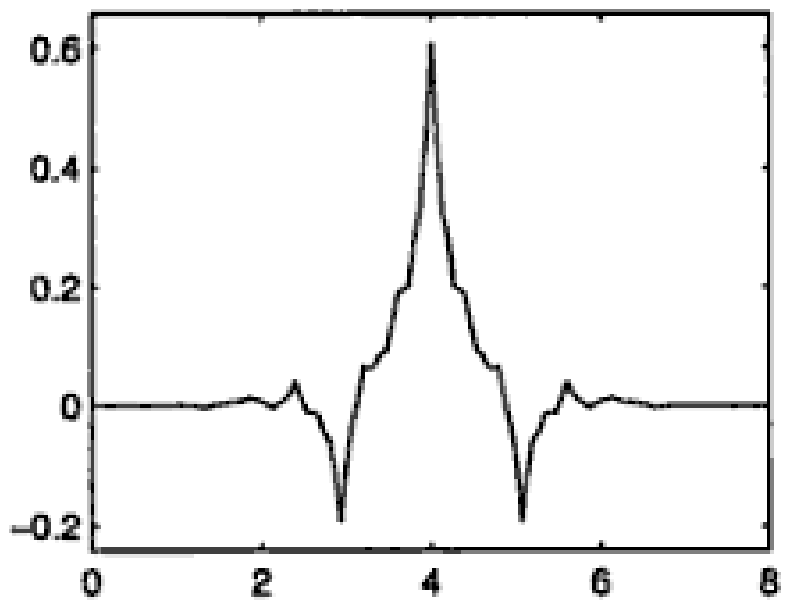

(a) Decomposition filter 


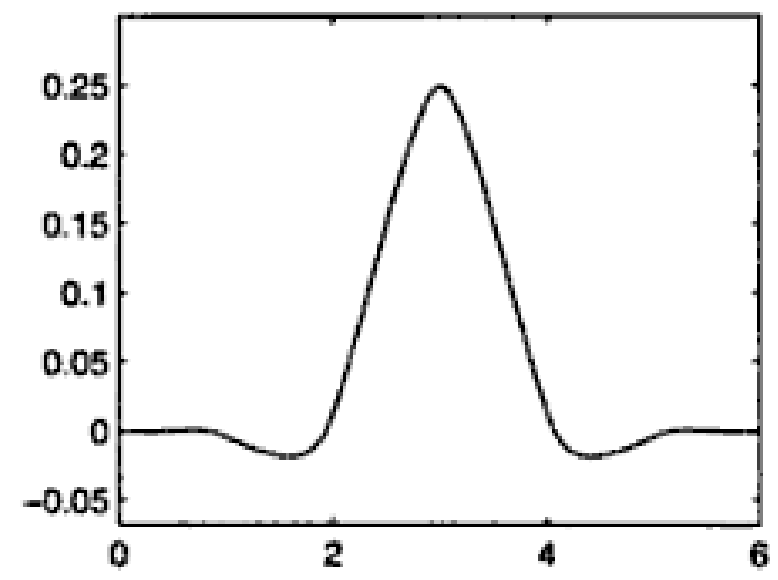

(b) Reconstruction filter

Figure 4.4 Decomposition and Reconstruction filters of a bi-orthogonal wavelet $\left(\mathrm{N}_{\mathrm{d}}=7\right.$, $\mathrm{N}_{\mathrm{r}}=9$ )

The first function dilates the input and the second reconstructs the data. The low pass and high passes filter dyadic coefficients decompose and reconstruct the wavelet of the signal X (t). Two higher levels of Bi-orthogonal Wavelets (Binlets) were constructed using theory described by Strang and Nguyen, 1996 in their famous book wavelets and filter banks. Both of the filters contained an array of thirteen low and high pass analysis coefficients $\left(\left\{h_{0}, h_{1}, h_{2}, \ldots h_{12}\right\}\right)$. The number of synthesis coefficients is different for the user defined functions named srik_h13/f7 and srik_h13/f11 build in MATLAB environment. Srik_h13/f7 function consists of seven low and high pass coefficients $\left(\left\{f_{0}, f_{1}, f_{2}, \ldots f_{6}\right\}\right)$, while srik_h13/f11 contained eleven low and high pass $\operatorname{coefficients}\left(\left\{f_{0}, f_{1}, f_{2}, \ldots f_{10}\right\}\right)$.

These filters were added to the wavelet tool box as user defined programs and used for wear monitoring of cutting tools, and to separate imperfections in weld joints. Srik_h13/f7 was used in the results section to perform the multisclae principle component 
analysis (MSPCA) of the signals. MSPCA was used to filter the original signal data and residuals at each level of decomposition to detect the deterministic changes in measurements (Bakshi, 1998). The Residual coefficients were compared to determine the wear level of the cutting tool and damages in the weld joints.

\subsection{Brief Summary of the Chapter}

This chapter described the data analysis techniques adopted to identify imperfections in cutting tools and welds. Lamb wave method along with the stransformation technique was demonstrated to analyze the wear level of a mill tool in Section 4.1. The procedure for data analysis using the surface response excitation was described in section 4.2. The last section explicated the binlet analysis that was used to separate dissimilar imperfections in weld joints. 


\section{CHAPTER 5}

RESULTS AND DISCUSSION: CHARACTERIZATION OF PIEZOELECTRIC MATERIALS AND ENERGY SCAVENGING

Instead of mathematical models, numerical and semi experimental computational tools were also used for the preliminary studies of the experiments conducted. The power generation of the bimorph materials was simulated by using the Finite Element Models (FEM) of the ANSYS package (Yenilmez et al., 2007). The Genetically Optimized Neural Network Systems (GONNS) were used for the estimating the optimum design of energy scavengers with the help of experimental data (Tansel et al., 2009) FEMs allowed us to work with any piezoelectric geometry without considering them as beam or plate. It was found that FEM based studies were very beneficial for studying attachment of piezoelectric patches to machine surfaces directly without using additional mass to the aerospace structures. GONNS was very useful for designing energy harvesters for restricted spaces based on experimental data.

Based on the experiments conducted in the Chapter Three of the dissertation, investigation was made with unprocessed signals to determine the dynamic and flaw detection characteristics of the piezoelectric actuators. Three different aspects related to the piezoelectric actuator efficiency are completely investigated in the following section of this chapter. This chapter will describe the dynamic characteristic of piezoelectric stripe actuators. It demonstrates the frequency response and nonlinear characteristics of these actuators. The results obtained from the rectangular and round shaped energy harvesters will be tested to determine their optimal electrical output due to periodical mechanical stress. 


\subsection{Frequency Response and Nonlinear Characterization}

The dynamic characteristics of the piezoelectric elements were initially studied under free vibration and without induced load. The energy harvester with single piezoelectric element was oscillated on the electromagnetic exciter showed in Figure 3.2 with a chirp wave and the response was analyzed on a signal analyzer. The frequency response of the piezoelectric actuator without any mass showed (Figure 4.1) a maximum magnitude at about $-18 \mathrm{~dB}$. The resonance frequency of the piezoelectric element was estimated as $1135 \mathrm{~Hz}$ without any mass.

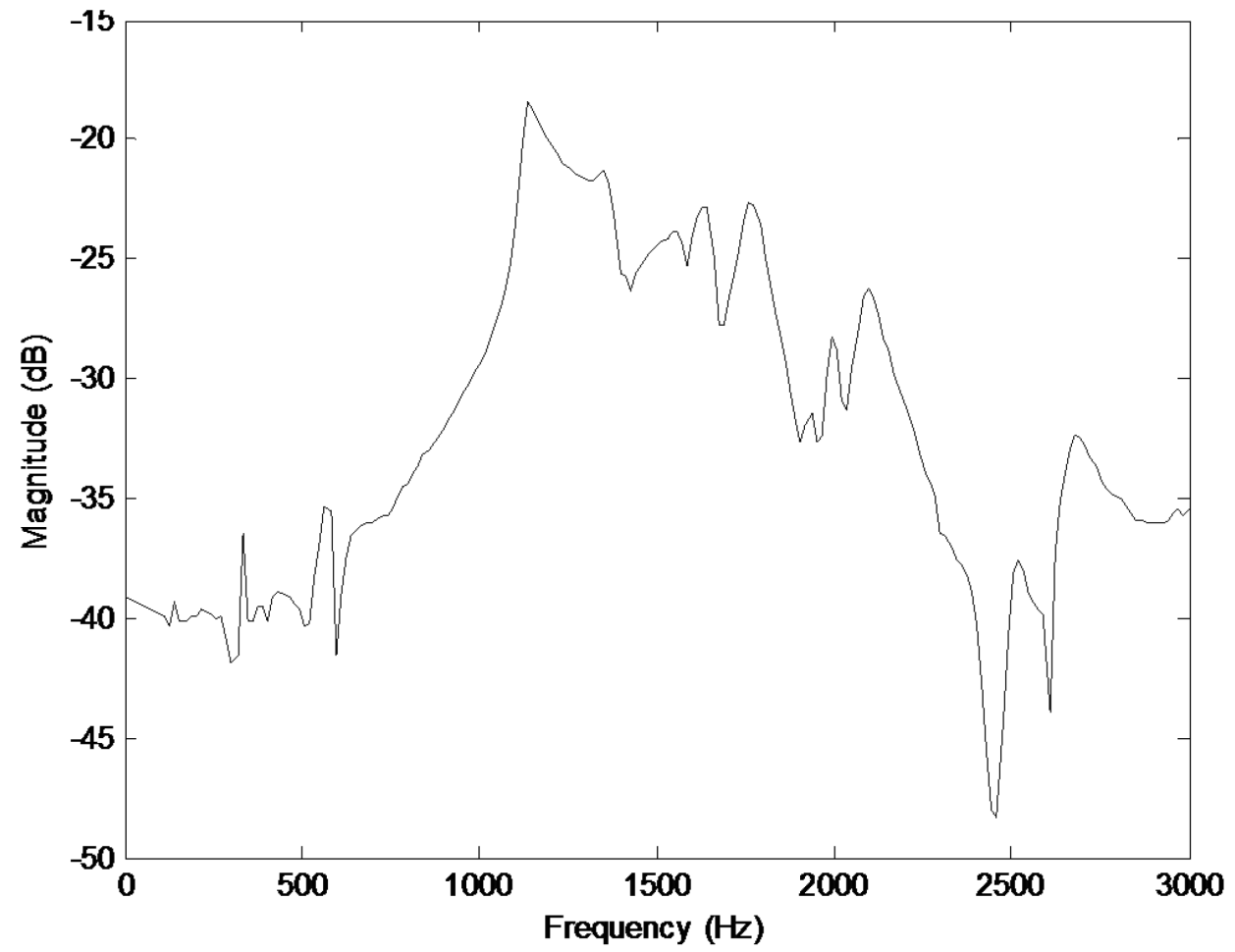

Figure 5.1 Frequency spectrum of single piezoelectric actuator without any weight 
The experiments on the VTS were conducted using forced vibration in order to derive an optimal energy of the device. Therefore, the resonance frequency was recalculated during forced vibration. An optimum weight (5.60 g) was used to induce a forced vibration in the design that generated maximum electrical energy. The same experiment described in the previous paragraph was repeated to calculate the natural frequency due to the effect of the optimum weight. It was observed that the natural frequency of the piezoelectric elements decreased due to the induced weight. To compare this present case with the previous case, it was assumed that half the weight $(2.85 \mathrm{~g})$ was carried by one of the two piezoelectric actuators. This development in the design setup caused a decrease in the frequency response to $107 \mathrm{~Hz}$ (Figure 4.2). The evaluation of the optimum frequency was used to estimate the electrical performance of the actuators (Section 4.1.2).

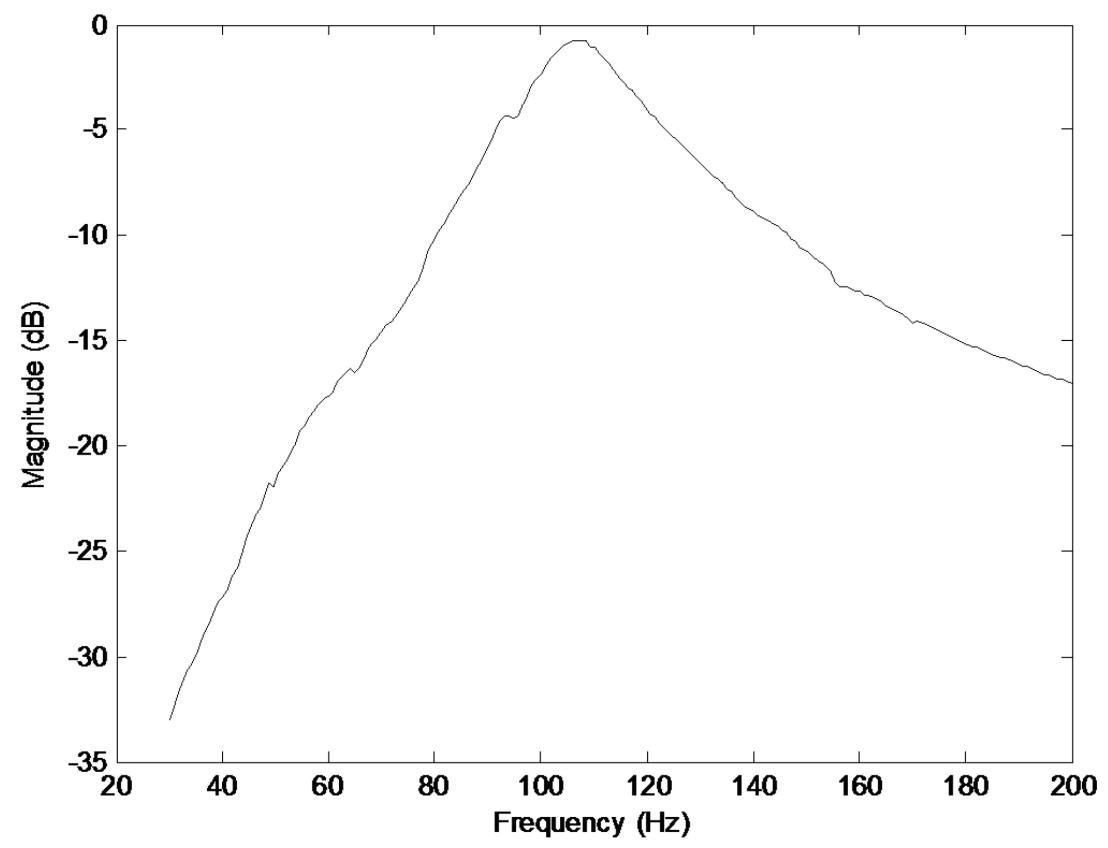

Figure 5.2 Frequency spectrum of a single piezoelectric actuator with half the weight $(2.85 \mathrm{~g})$ 
The investigation was further continued to determine the output characteristics of the actuators around the optimum frequency calculated in the last paragraph (107 HZ). The linearity of the actuators was estimated at different frequencies $(40 \mathrm{~Hz}-108 \mathrm{~Hz})$ of vibration using the function generator. The results showed highly nonlinear AC outputs during all excitation frequencies Figure 4.3. The composite signal was prepared by putting together the signals collected at the $40,50,60,70,80,90$ and $108 \mathrm{~Hz}$ vibration frequencies. Each 0.1 second long section was collected at one of the listed frequencies.

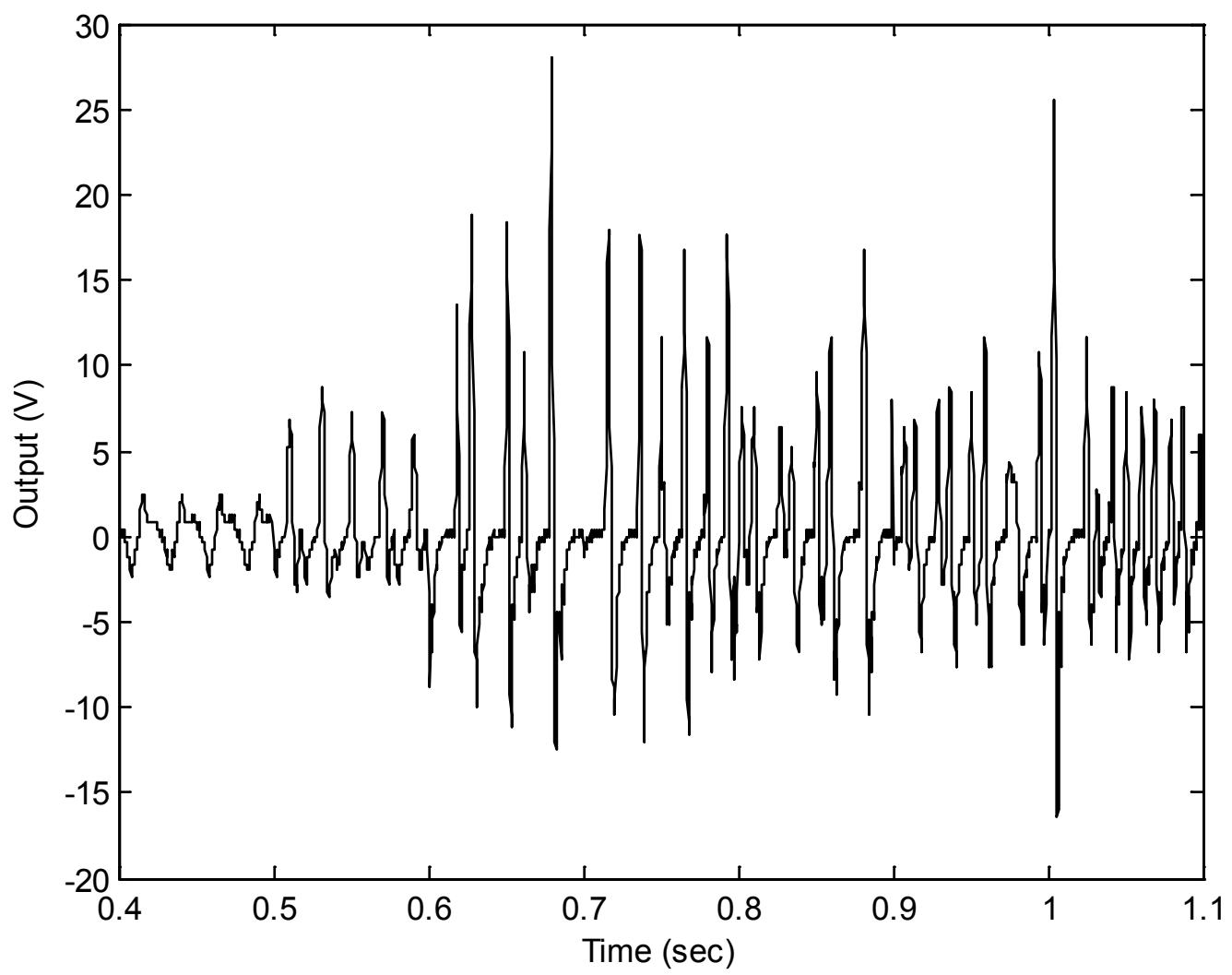

Figure 5.3 Non Linear output characteristics of piezoelectric actuators: 0.4-0.5, 0.5-0.6, $0.6-0.7,0.7-0.8,0.8-0.9,0.9-1$, and $1-1.1$ second intervals corresponds to outputs at the 40, 50, 60, 70, 80, 90, and $108 \mathrm{~Hz}$ oscillations 


\subsection{Electrical Response due to Harmonic Excitation of Actuators}

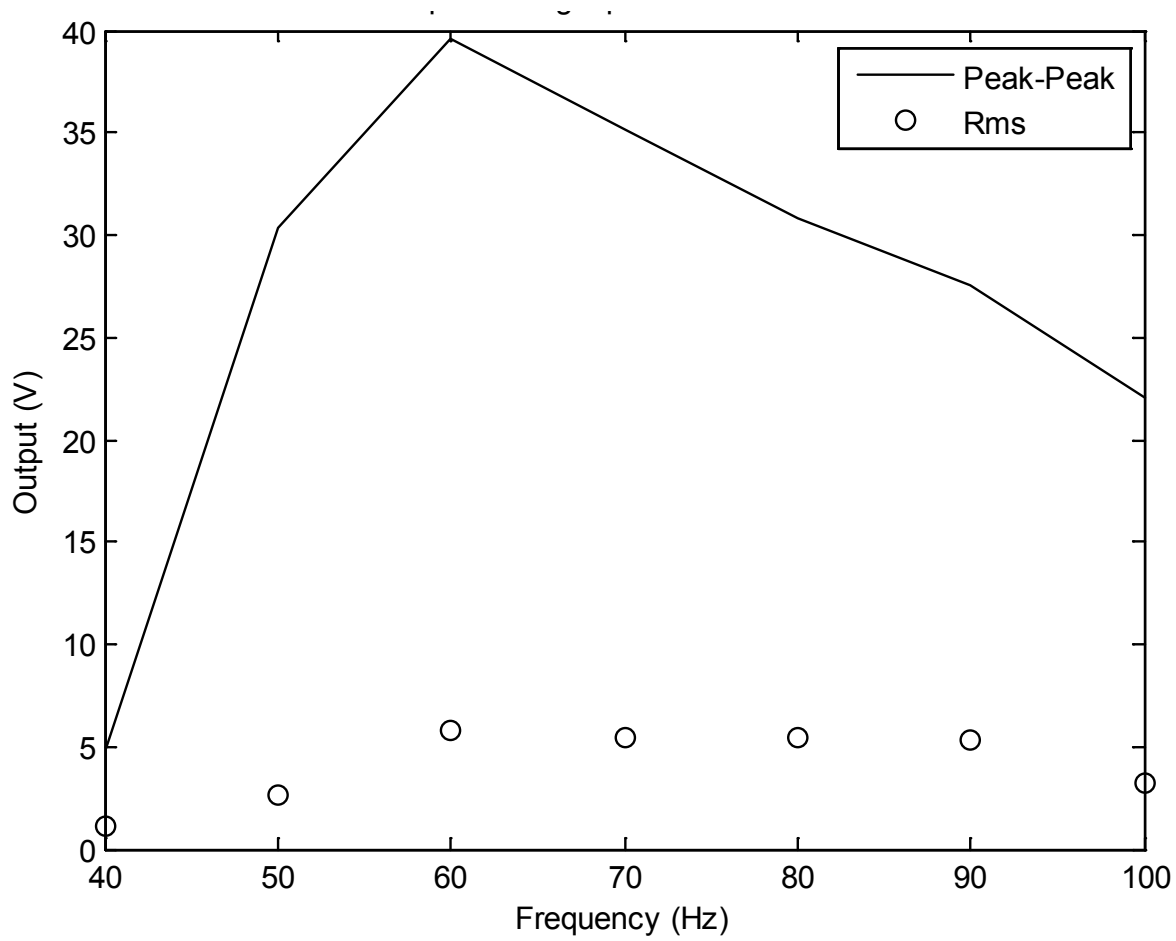

Figure 5.4 Comparisons of RMS and Peak-Peak output values

Since the nonlinear characteristics of the actuators were evident from Figure 4.3, conclusions were made using the peak to peak and the Root Mean Square (RMS) output estimation. The Peak-Peak and RMS values obtained from the Figure 4.4 indicated that the optimum output of the actuators could be achieved around $50-60 \mathrm{~Hz}$. The highest values of the output were obtained at $60 \mathrm{~Hz}$ frequency. Although the mass was almost twice the one used for the previous tests for identification of the dynamic characteristics of the piezoelectric elements, two elements were supporting the mass in the harvester. The final experiments were performed at $50 \mathrm{~Hz}$ since it corresponds to $3,000 \mathrm{rpm}$ and there are many mechanical systems operating around this frequency. The chances of 
device failure to a greater extent were also observed when tested at higher frequencies (above $50 \mathrm{~Hz}$ ).

A two element compact size device was developed (Section 3.1.3) using these actuators to record the maximum generated power. The newly developed compact device was connected to digital oscilloscopes with $1 \mathrm{M} \Omega$ impedance. The DC voltage output of the energy harvester rose to $10 \mathrm{~V}$ in less than 10 seconds when it was excited at $50 \mathrm{~Hz}$ as shown in Figure 4.5, and when the amplitude was about $3.2 \mathrm{~mm}$ based on the trace of the oscillation left on a paper. It was observed that Voltage continued to increase and reached 14 Volts in less than 2 minutes. Voltage continued to rise and reached over $16 \mathrm{~V}$ in less than 3 minutes. The output oscillated during the experiments due to the nonlinearity in the device design. Based on the impedances of the data acquisition devices the power output of the energy harvesters was $100 \mu \mathrm{W}$ in less than 10 seconds and $196 \mu \mathrm{W}$ in 2 minutes. The highest observed power in the experiment was above $250 \mu \mathrm{W}$.

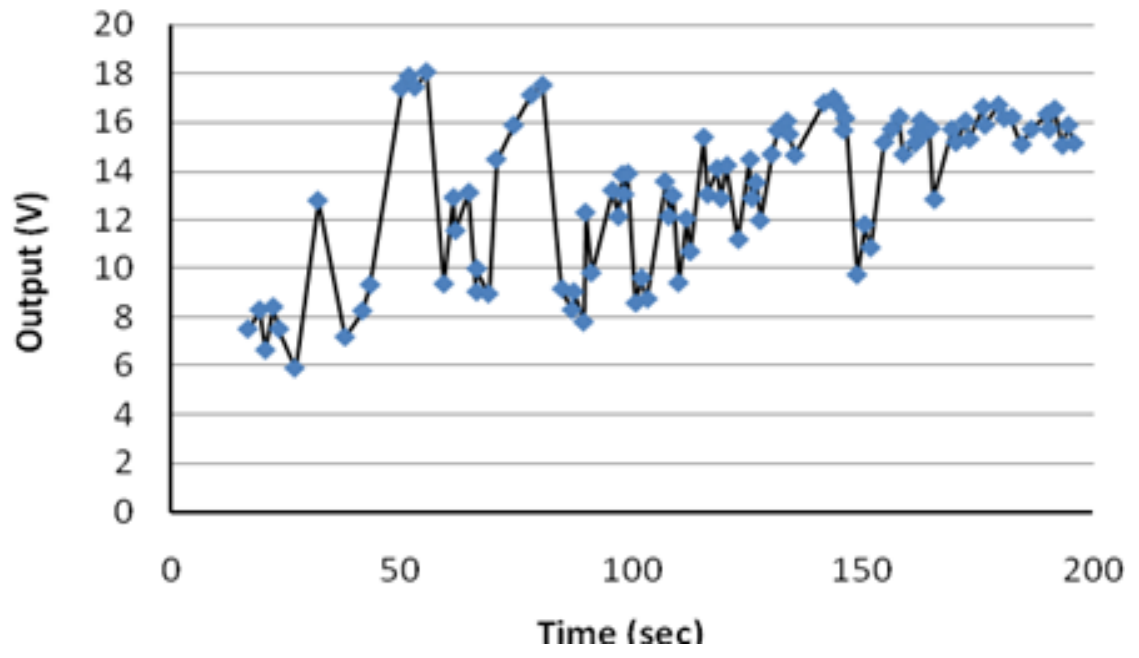

Figure 5.5 Electrical response of the energy harvester (Round shape) 
A better design of the device was accomplished using rectangular aluminum case, which facilitated a better movement of weight inside, and reduced the nonlinear characteristics of the output. The harvesters with square cross-section generated voltages between 10 and 14 volts when excited at $50 \mathrm{~Hz}$. The test was conducted at approximately the same amplitude of vibration where round cross-section harvesters were tested. The design of the mass was slightly changed to fill the available space of the square crosssectional area better. Also the notch of the mass was slightly sharpened to give it V shape to have bigger weight. The weight of the modified masses increased to 5.1912 gram ( $\sim 5.60$ g, optimum weight for the design). The modified energy harvester generated $15 \mathrm{~V}$ when excited at $50 \mathrm{~Hz}$ with $4.5 \mathrm{~mm}$ amplitude. The output voltage increased to $25 \mathrm{~V}$ when the amplitude was increased to $9 \mathrm{~mm}$ (Figure 4.6).

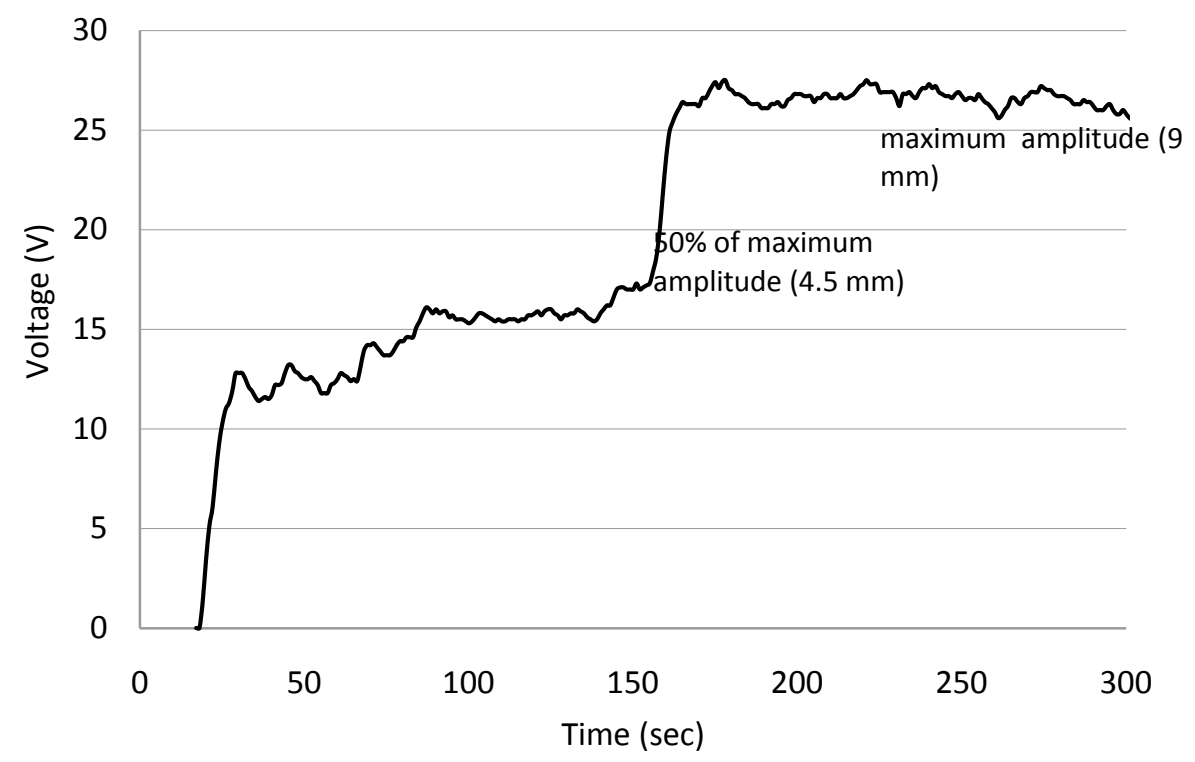

Figure 5.6 Electrical response of the energy harvester (Rectangular design) 
The output of different sized energy generators fabricated in our laboratory is listed in Table 5.1. Historically; the 2nd generation energy harvester $(7.9 \mathrm{~cm} \times 7.9 \mathrm{~cm} \times 3$ $\mathrm{cm})$ generated $1740 \mu \mathrm{W}$ with a power density of $9.43 \mu \mathrm{W} \mathrm{cm}-3$. The output of the third generation energy harvester with round cross-sectional area was $256 \mu \mathrm{W}$ at $50 \mathrm{~Hz}$ frequency (Tansel et al., 2008). The energy generator with square cross-section was robust enough to vibrate at higher amplitudes and generated $625 \mu \mathrm{W}$ at $50 \mathrm{~Hz}$. The power density was $57.89 \mu \mathrm{W} \mathrm{cm}-3$. The energy harvester volume and power density variations trend of the listed harvesters are showed in Figure 5.7.

Table 5.1 List of different generation energy harvesters

\begin{tabular}{lcccc}
\hline $\begin{array}{l}\text { Energy harvesting } \\
\text { device }\end{array}$ & $\begin{array}{c}\text { Test conditions } \\
\text { (frequency and } \\
\text { approximate } \\
\text { amplitude) }\end{array}$ & $\begin{array}{c}\text { Size - } \\
\text { volume } \\
\left(\mathrm{cm}^{3}\right)\end{array}$ & $\begin{array}{c}\text { Power } \\
(\mu \mathrm{W})\end{array}$ & $\begin{array}{c}\text { Power density } \\
\left(\mu \mathrm{W} \mathrm{cm}^{-3}\right)\end{array}$ \\
\hline $\begin{array}{l}\text { Third generation } \\
\text { with square cross- } \\
\text { sectional area }\end{array}$ & $50 \mathrm{~Hz}, 0.9 \mathrm{~cm}$ & 10.79 & 625 & 57.89 \\
$\begin{array}{l}\text { Third generation } \\
\text { with round cross- } \\
\text { sectional area }\end{array}$ & $50 \mathrm{~Hz}, 0.4 \mathrm{~cm}$ & 7.7 & 256 & 33.24 \\
$\begin{array}{l}\text { Second } \\
\text { generation energy } \\
\text { harvester }\end{array}$ & $\begin{array}{l}60 \mathrm{HZ}, 0.325 \\
\mathrm{~cm}\end{array}$ & 184.4 & 1740 & 9.43 \\
\hline
\end{tabular}




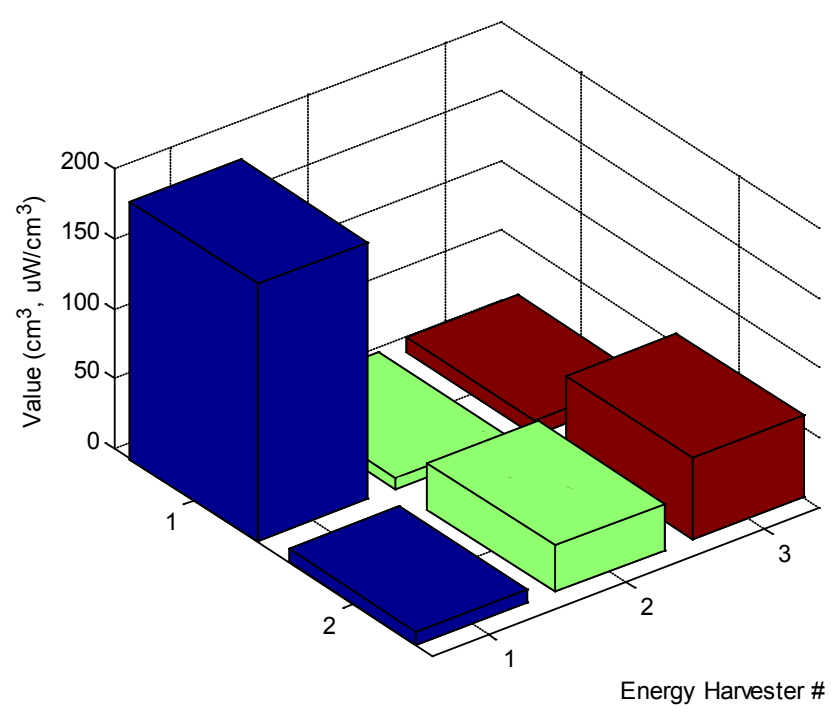

Figure 5.7 Comparison of Volume and Power Density of energy harvesting devices

\subsection{Brief Summary}

The dynamic characteristics, and electrical output obtained from piezoelectric stripe actuators, were described in this Chapter. Frequency response and nonlinear characterization of the piezoelectric actuators was revealed, and the optimum frequency to achieve maximum electrical response was evaluated in Section 5.1. The electrical response of two differently designed energy harvesting devices was evaluated, and compared in Section 5.2. It was observed that the rectangular shape harvester generated maximum power output. 


\section{CHAPTER 6}

\section{RESULTS AND DISCUSSION: IDENTIFICATION OF DEFECTS OF COMPLEX}

\section{SHAPES AND WELDS}

This section presents the results and discussion of the experiments conducted on end mill and weld joint. The experimental Setup of these results was described in Chapter Three. The analysis was performed using Lamb wave method, SuRE Techniques and biorthogonal wavelet (Binlet) analysis. The procedures for data analysis were demonstrated in Chapter Four.

\subsection{Wear Estimation of an End mill}

\subsubsection{Lamb wave Analysis}

The Lamb wave perturbations at the receiving actuator were recorded on a computer during the five stages of wear. Initially, the no wear condition data was recorded in order to compare the initial signal with the signals at different stages of wear.
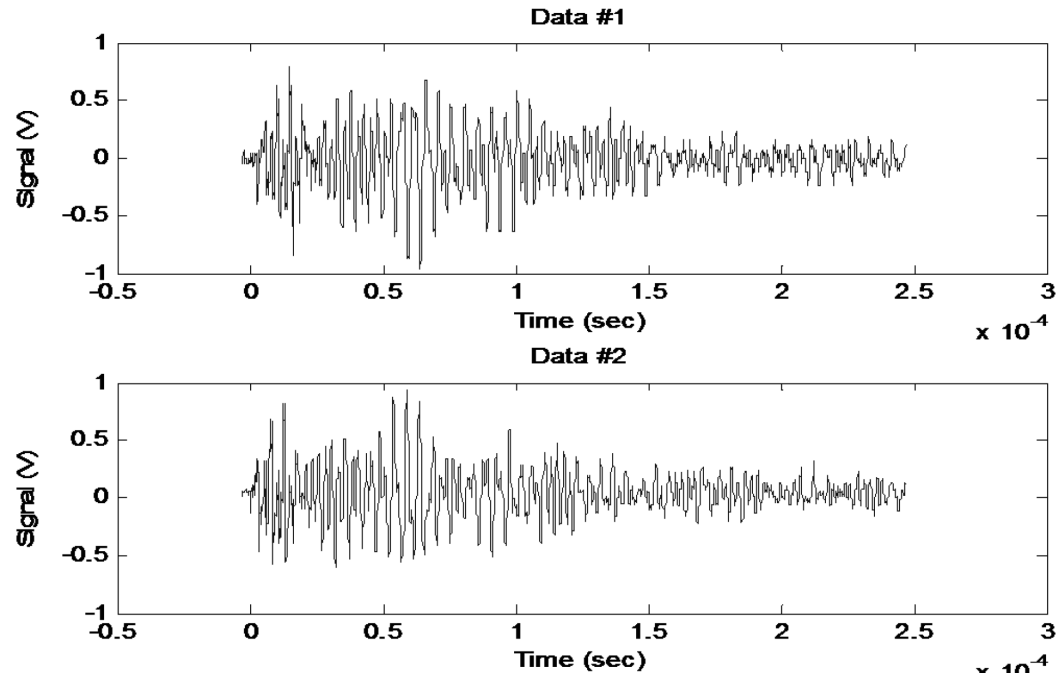

(a) no wear vs. slightly worn 

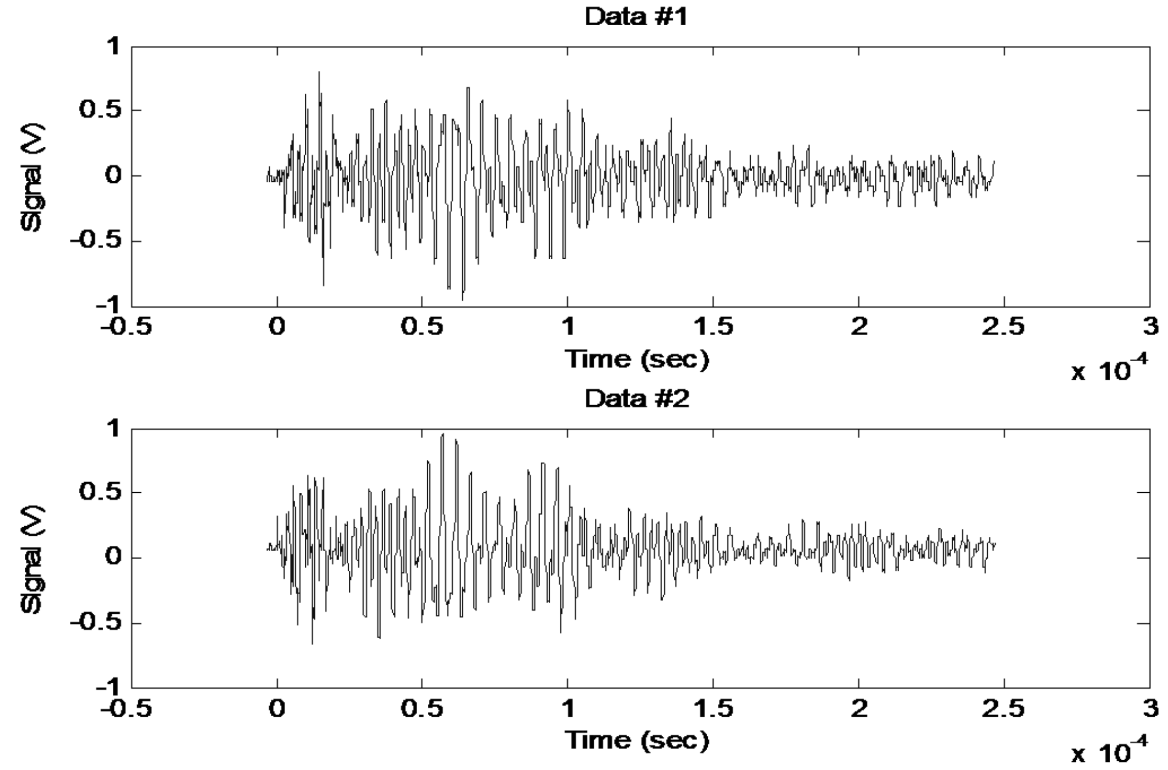

(b) no wear vs. $1^{\mathrm{s}}$ medium wear
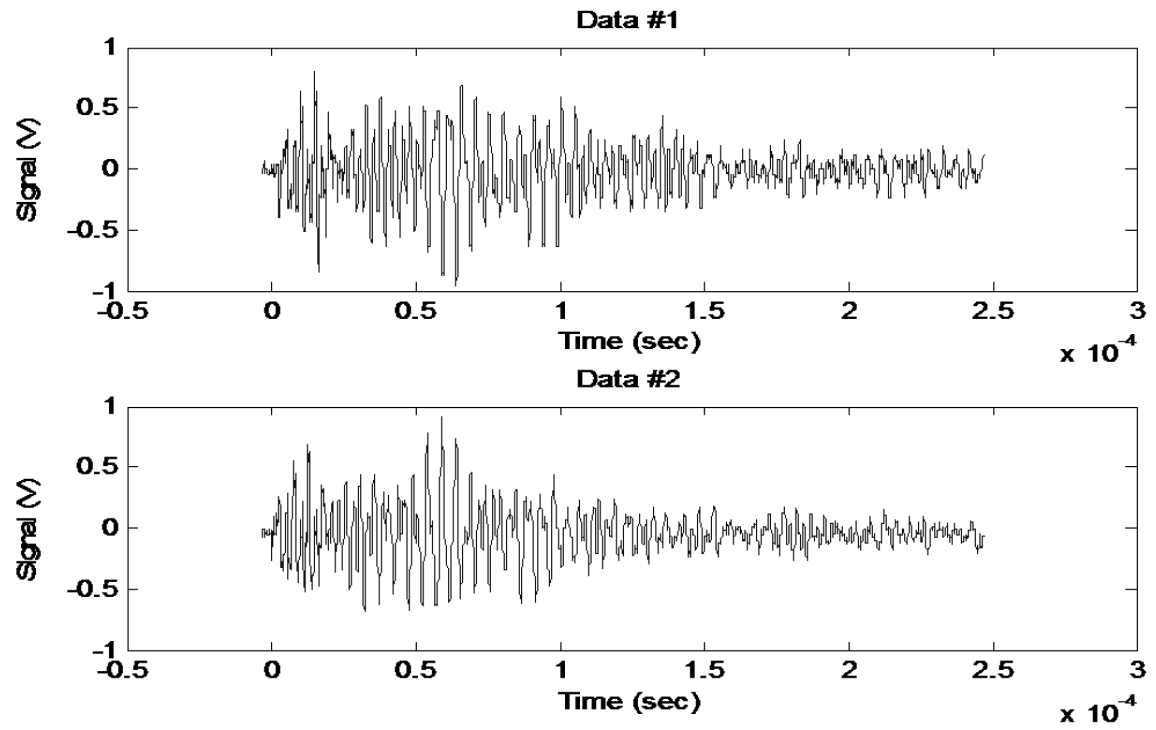

(c) no wear vs. heavy wear 

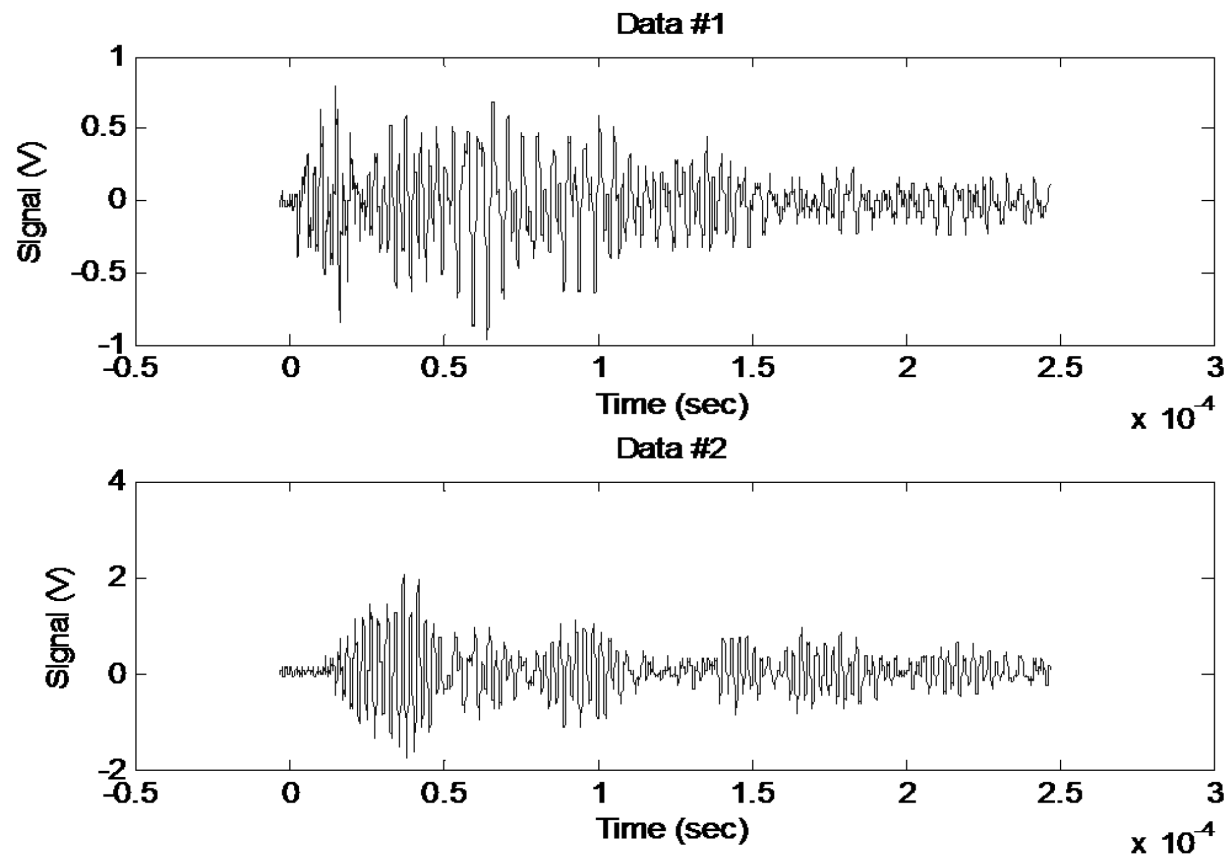

(d) no wear vs. severe wear
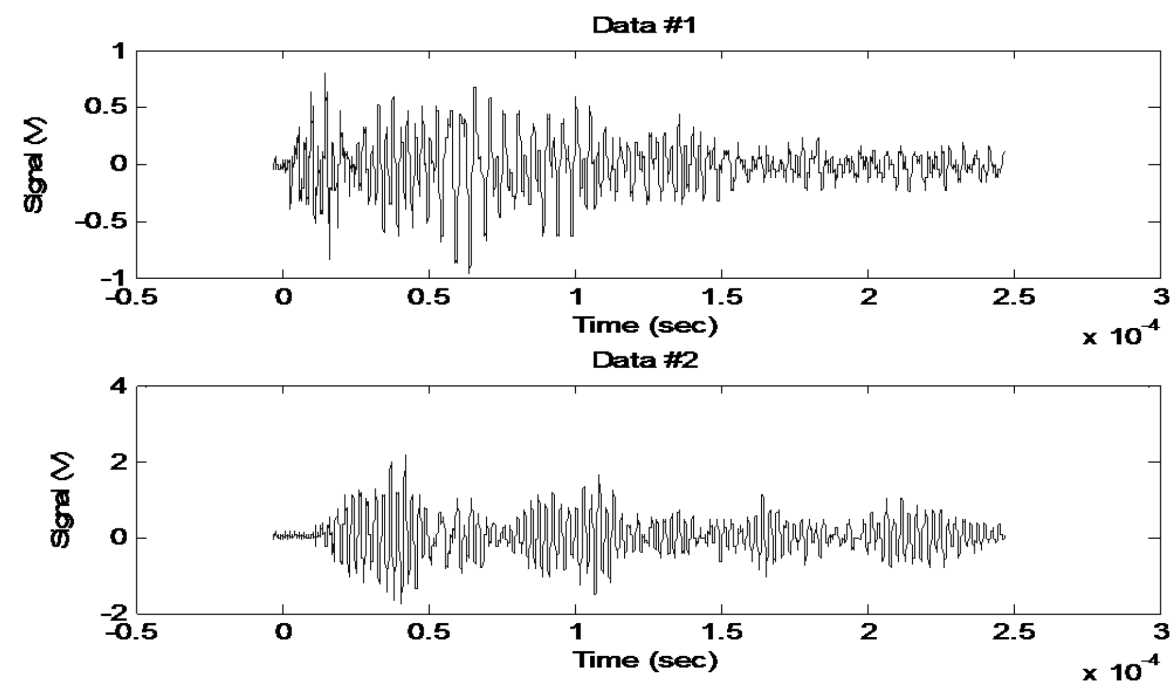

(e) no wear vs. extreme wear

Figure 6.1 Comparison of Data 1 (no wear signals) with Data 2: (a) Slightly worn (b) Medium wear(c) Heavy wear (d) Severe wear (e) Extreme wear of mill tool 
Data 1 described the no wear condition of the signal, and Data 2 represents the wear condition in Figure 6.1, which also demonstrates the comparison of wear during the five stages with the wear. The Lamb waves perturbations obtained in these time domain signals were not sufficient to determine the wear level. In order to estimate the progressive wear of the mill tool over time, an artificial wear was induced a tool is induced in five steps. A MATLAB program was used to calculate the envelopes of the signals by using s-transformation and presented in Figure 6.2. (a) (c) (d). The Stransformation for the micro tool is showed in Figure 6.3

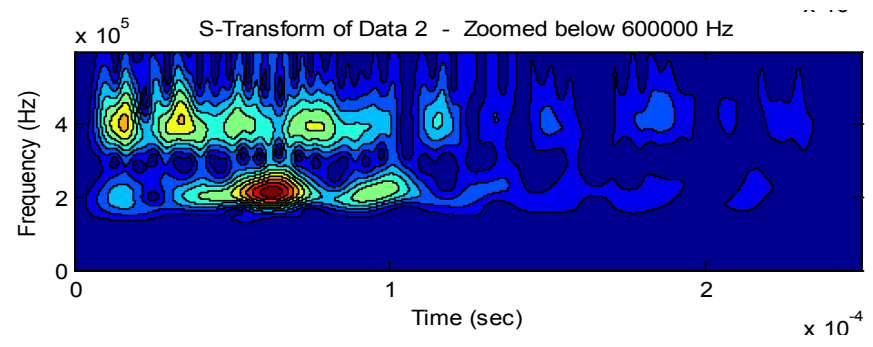

(a)

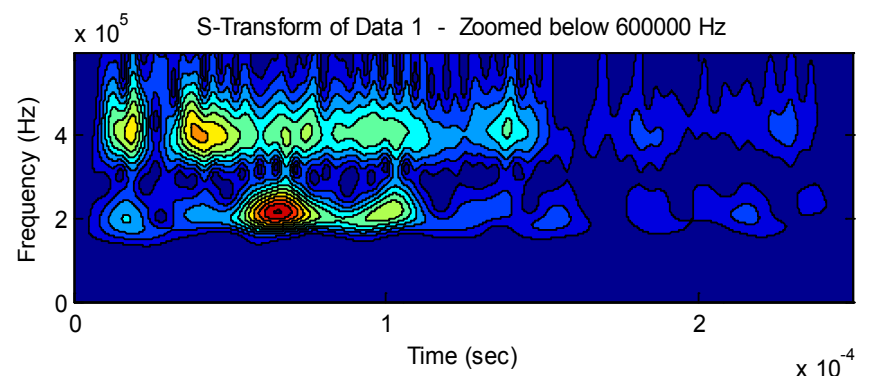

(b)

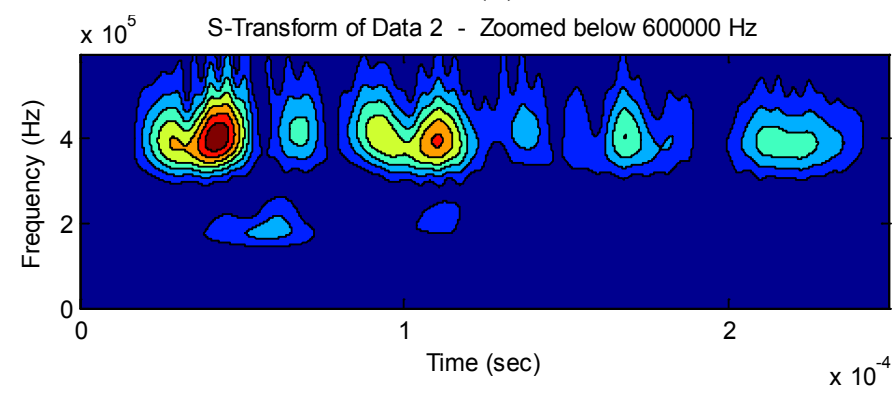

(c) 


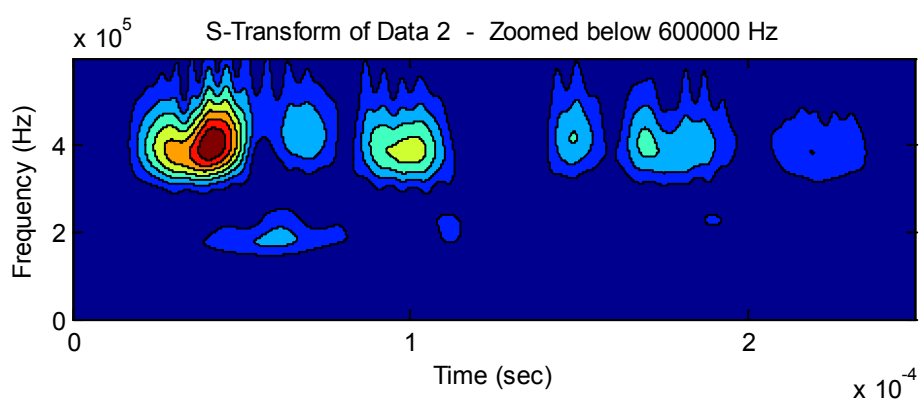

(d)

Figure 6.2 Comparison of s-transformation data zoomed below $600 \mathrm{KHz}$ (a) No wear (b) Slightly worn (c) Heavy wear (d) Extreme wear

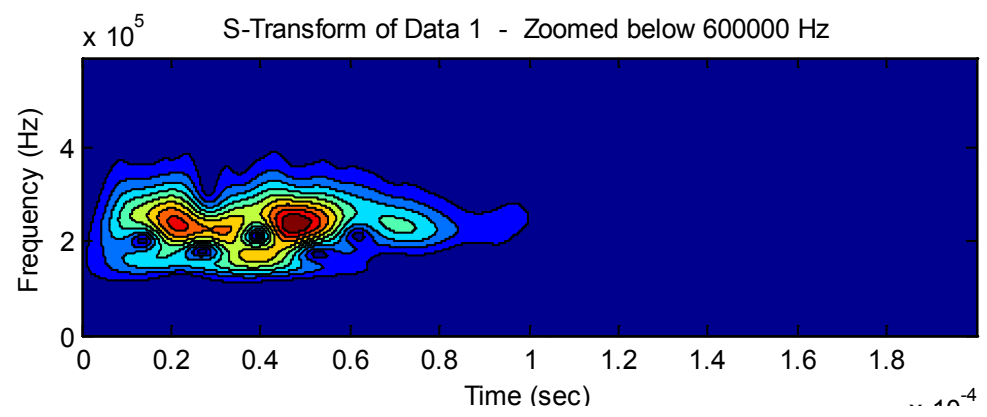

(a)

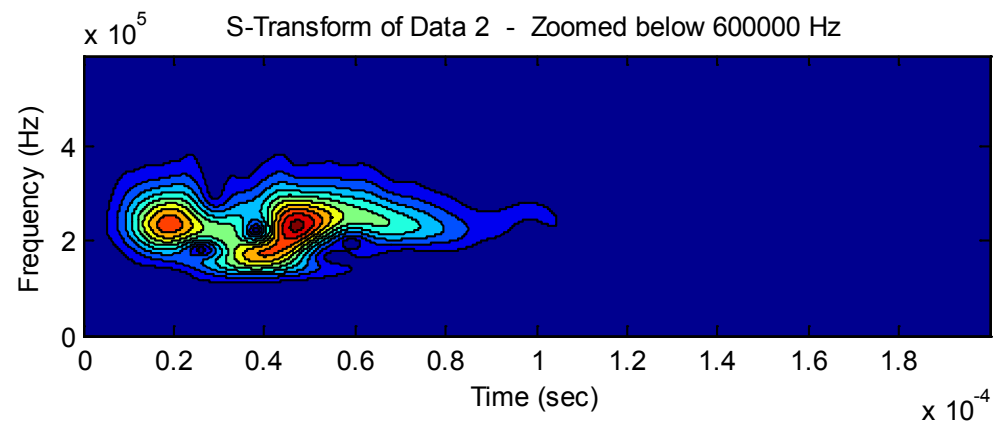

(b) 


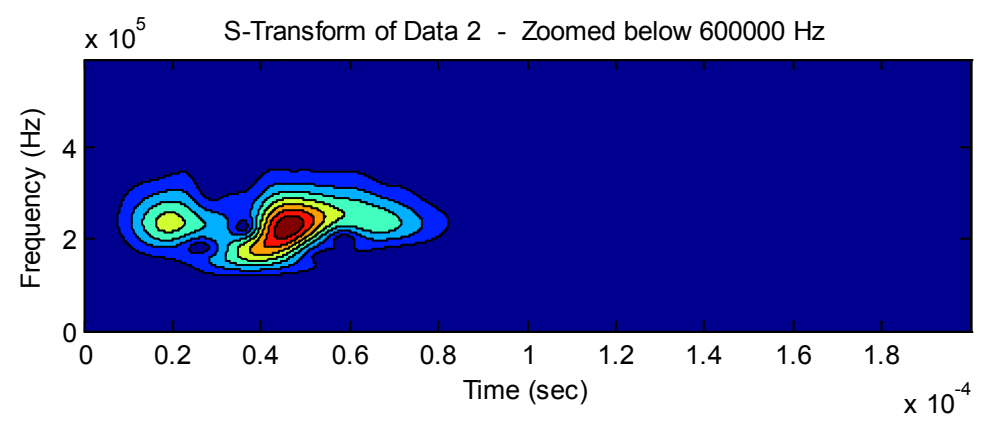

(c)

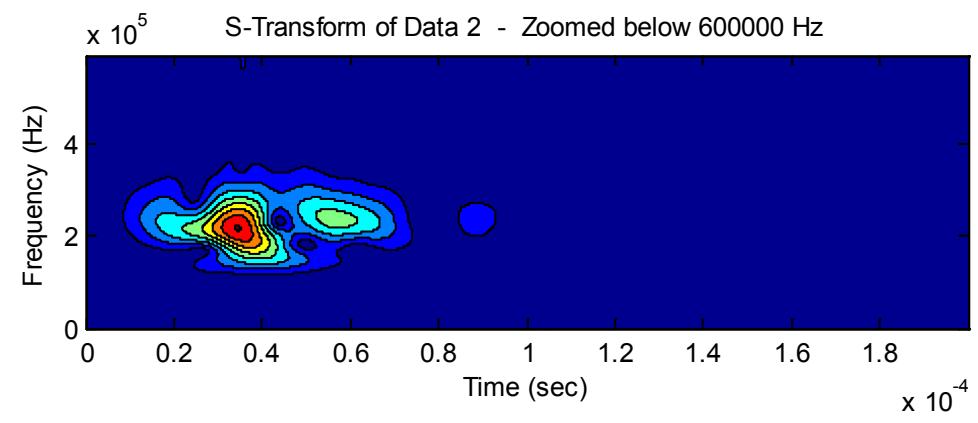

(d)

Figure 6.3 Comparison of s-transformation of Micro tool (a) No wear (b) Slightly worn (c) Heavy wear (d) Extreme wear

In order to achieve the task, The s-transformations of the recorded Lamb wave perturbations were zoomed below $600 \mathrm{kHz}$ frequency and analyzed. The results obtained during this study are showed in Figure 6.2. The observation apprises the small localized changes in low wear (Figure 6.2 (a)) pattern when compared to the original envelope (Figure $6.2(\mathrm{~b})$ ). The transition from no wear to severe wear was keenly observed during the analysis (Figure 6.2 (d)). Lamb waves travelled through the tool medium, and transmitted back after hitting the surface, and this was the reason for retaining a difference in envelope profile at the receiving sensor. The characteristics of the $\mathrm{S}$ transform and envelope in Figure 6.2 and Figure 6.3 were changed particularly while the 
wave propagation characteristics changed with material loss created by wear. A similar trend of the S-Transformation was also observed in case of the micro tool.

The envelopes of the signal were presented in Figure 6.4 (a), (b), (c), (d), (e) which classified wear level more accurately. In each case envelopes of the signal at different stages of wear were compared to the original signal. The results taken at 200 $\mathrm{kHz}$ isolated frequency were reasonable, and the amplitude of the Lamb wave was changing in a pattern due to increase in wear. The tool envelopes of the micro tool are compared in figure 6.5 (a) (b) (c) (d) (e).

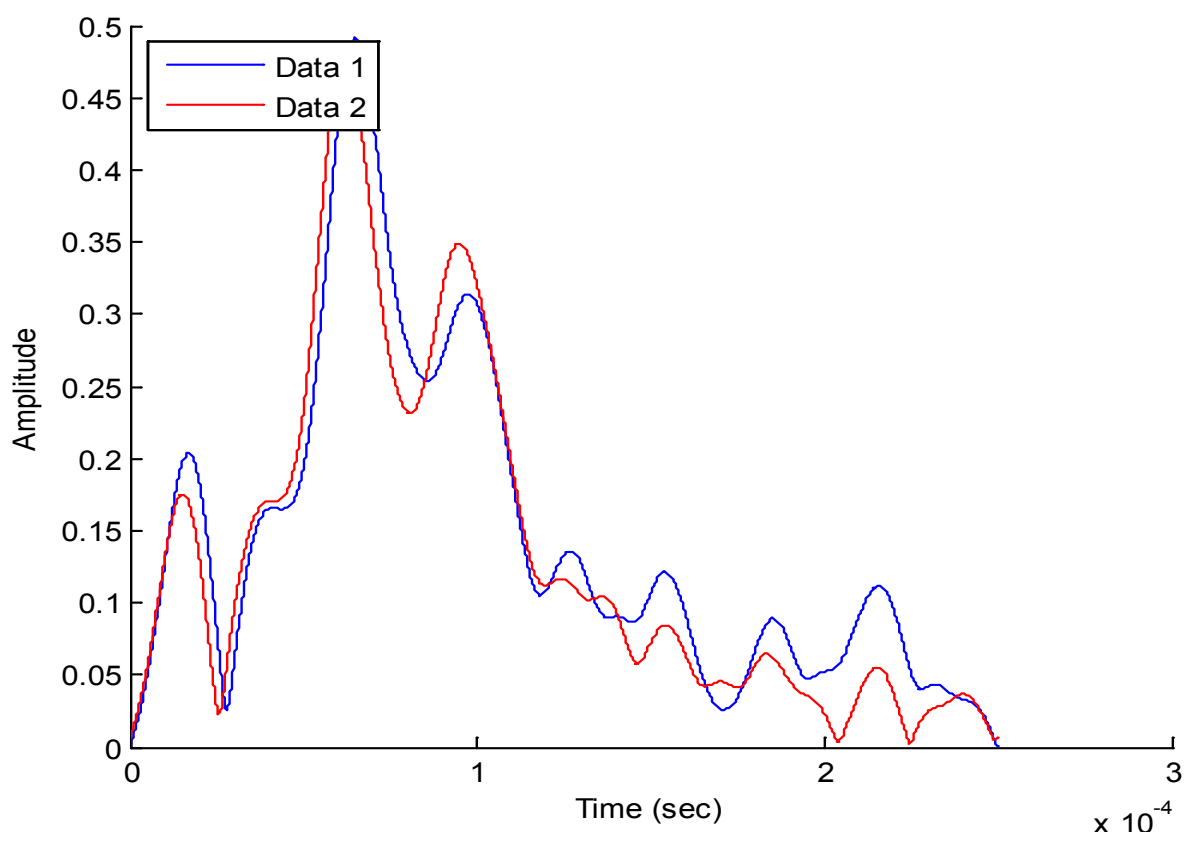

(a) no wear vs. slightly worn 


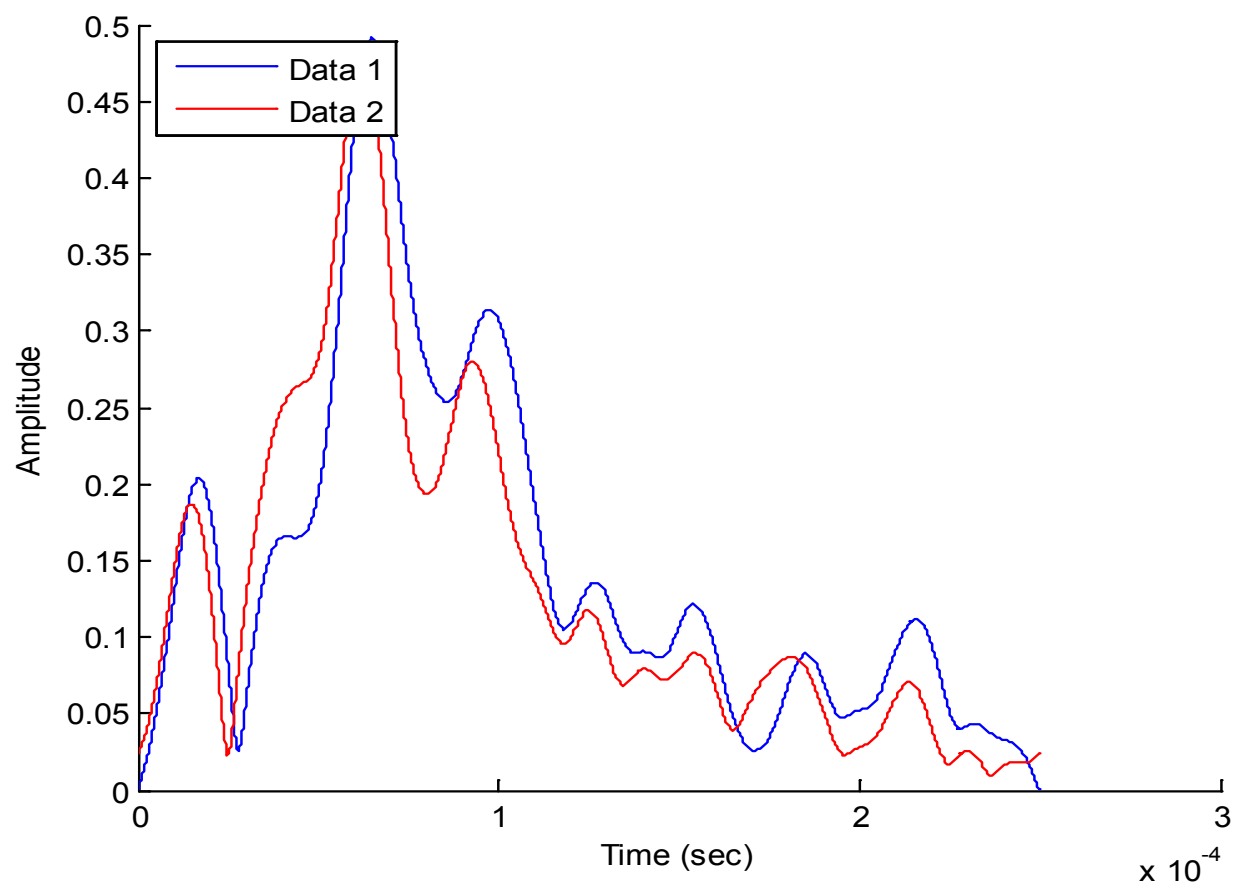

(b) no wear vs. medium wear

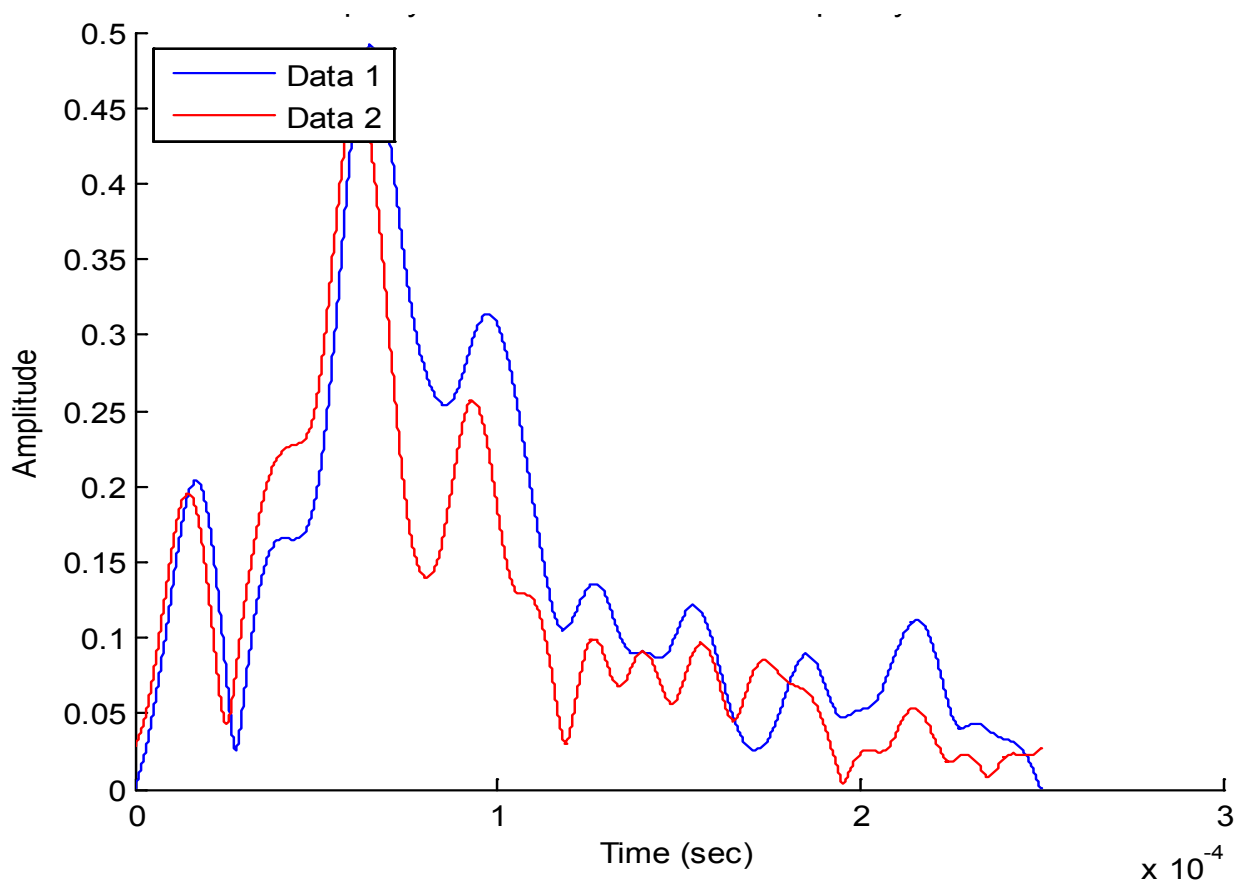

(c) no wear vs. heavy wear 


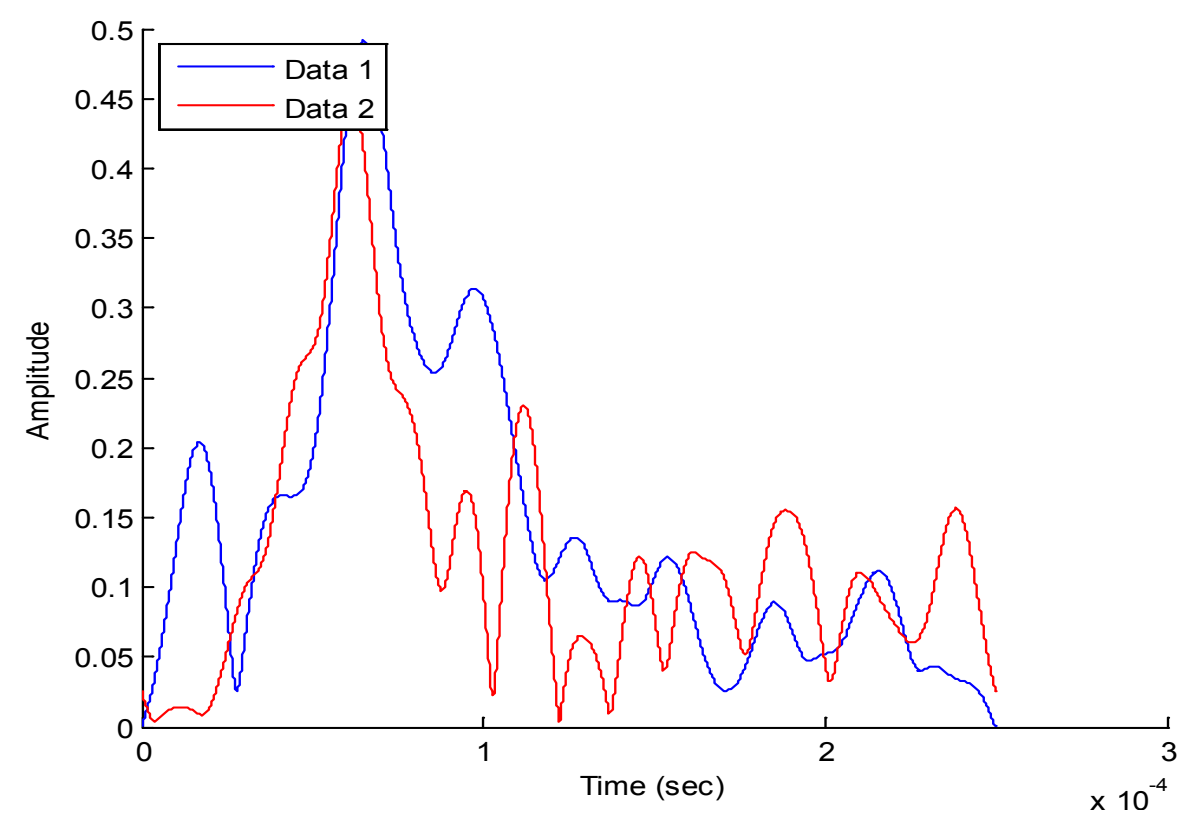

(d) no wear vs. extreme wear

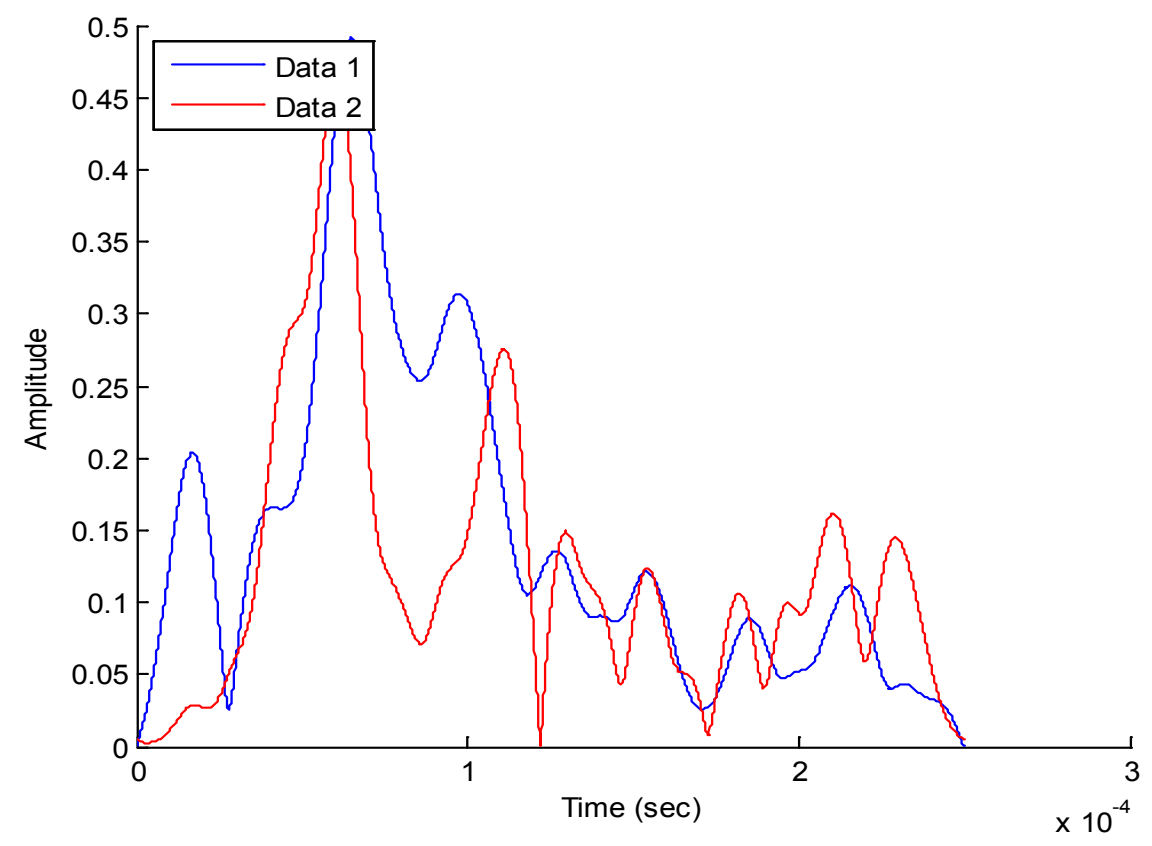

(e) no wear vs. severe wear

Figure 6.4 Comparison of s-transformation envelopes during five stages of wear (a) Slightly worn (b) medium wear (c) Heavy wear (d) Severe Wear (e) Extreme wear of mill tool 


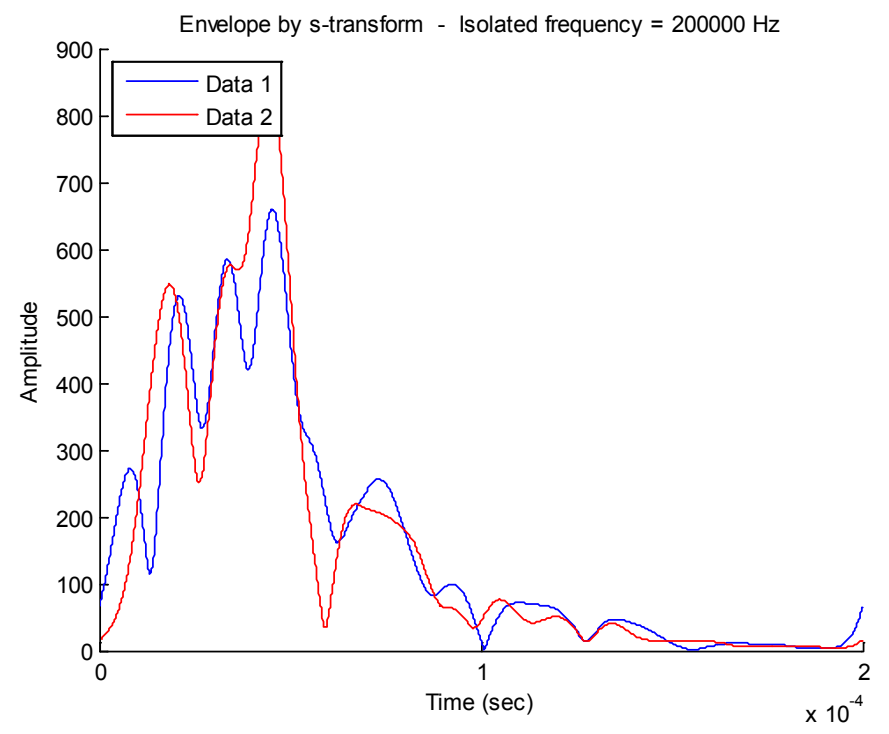

(a) no wear vs. slightly worn

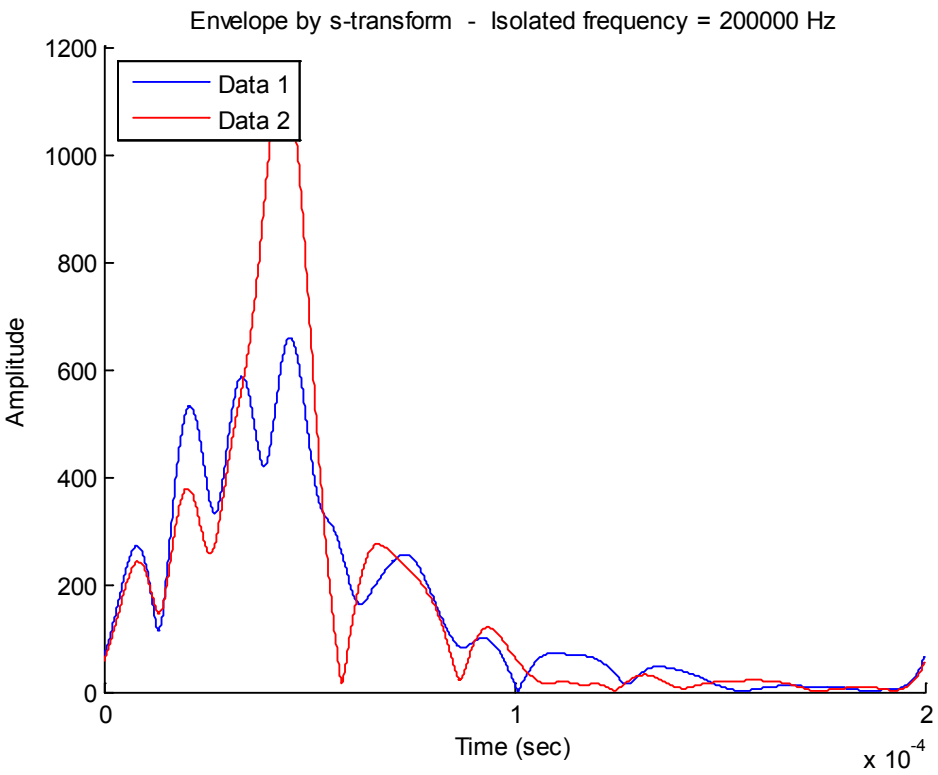

(b) no wear vs. medium wear 


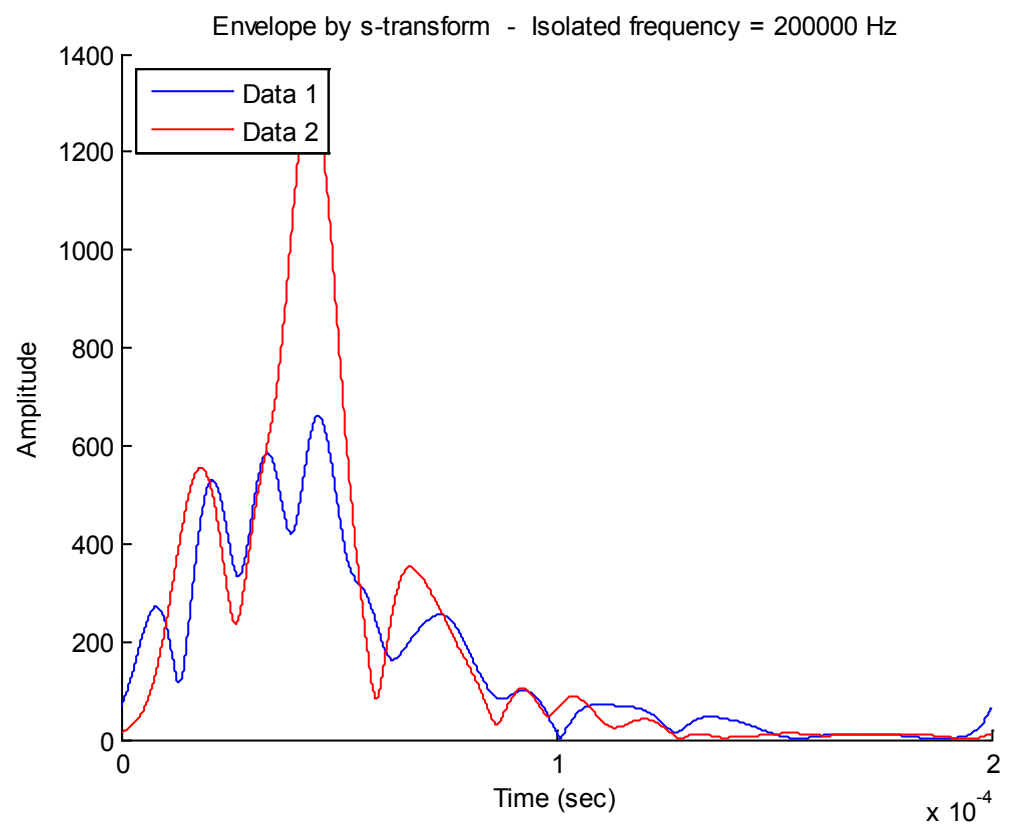

(c) no wear vs. heavy wear

(d)

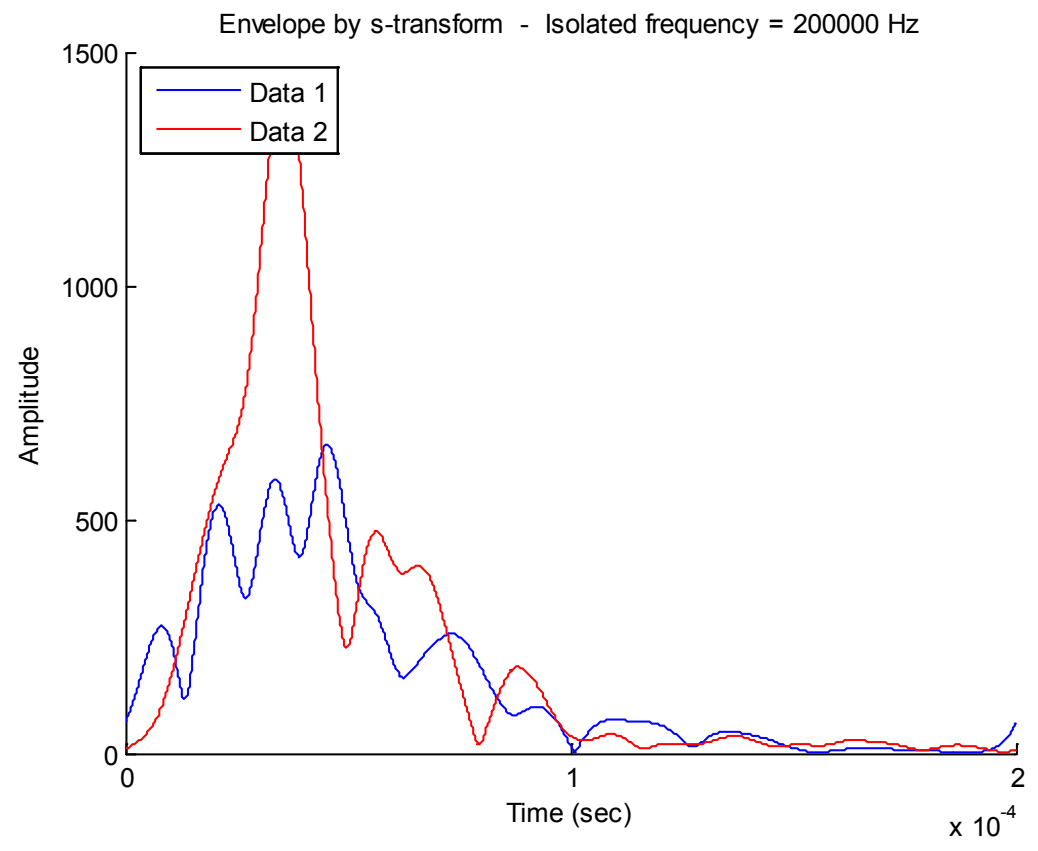

(d) no wear vs. severe wear 


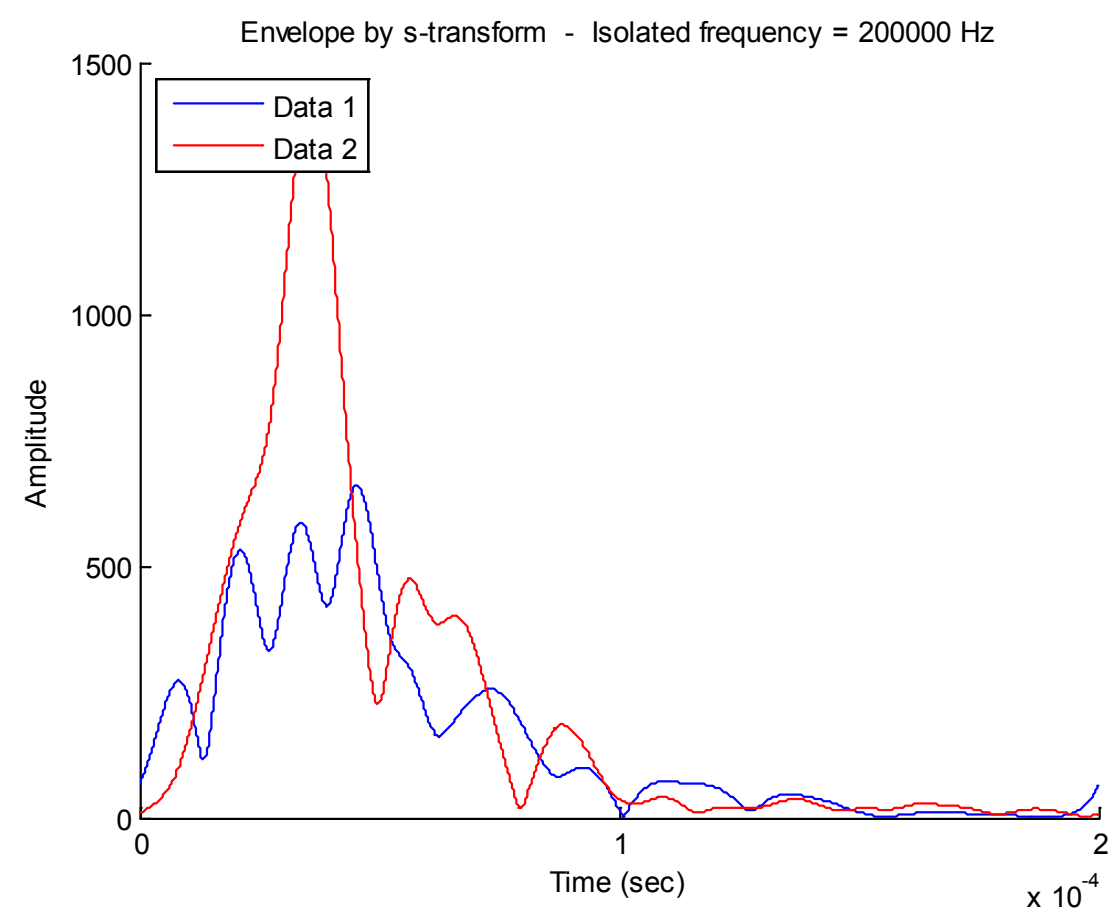

(e) no wear vs. extreme wear

Figure 6.5 Comparison of s-transformation envelopes of the micro tool (a) Slightly worn (b) medium wear (c) Heavy wear (d) Severe Wear (e) Extreme wear of mill tool

The envelopes of the s-transformation indicated that the amplitude of the highest peak deviated from the original envelope until medium wear (Figure 6.4 (c)), and directional change in the perturbations were observed due to the severe wear. Datal in each of the graphs of figure 6.4 and 6.5 represents s-transformation envelope taken when there was no wear. Data 2 indicates the wear at each level of the five stages as described in Chapter Three. 
The square of the difference between the time domain observations of different wear levels and a baseline was studied by using a Simulink model (Figure 6.6). The excitation frequency was $200 \mathrm{kHz}$. The squares of the differences were presented in Figure 6.7. The first cut in the figure corresponds to a low level wear of the tool. Second and third cuts represent a medium wear level. Final cut indicates a sever wear at the tip of the mill tool.

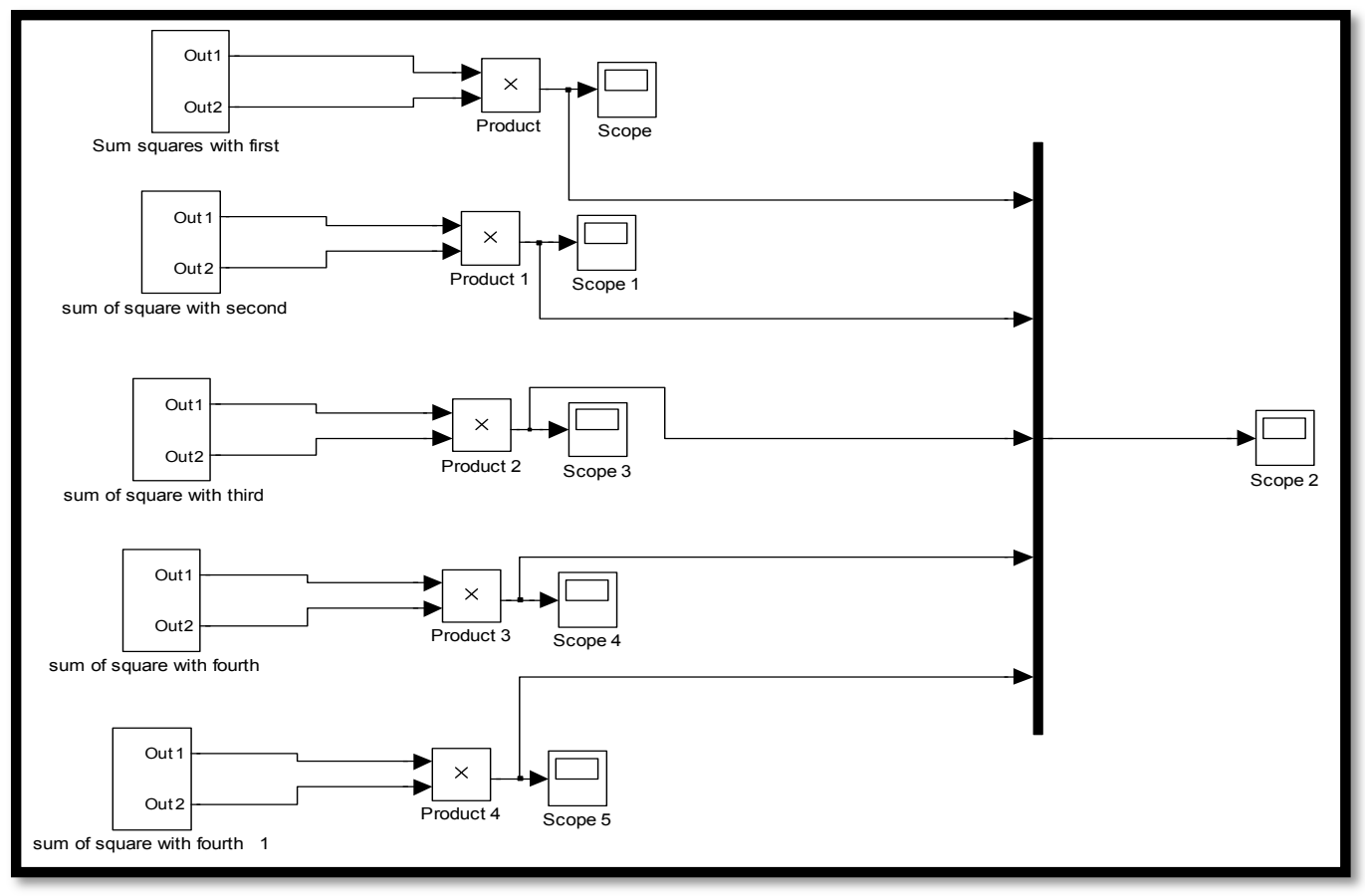

Figure 6.6 Simulink model to evaluate square of difference value 


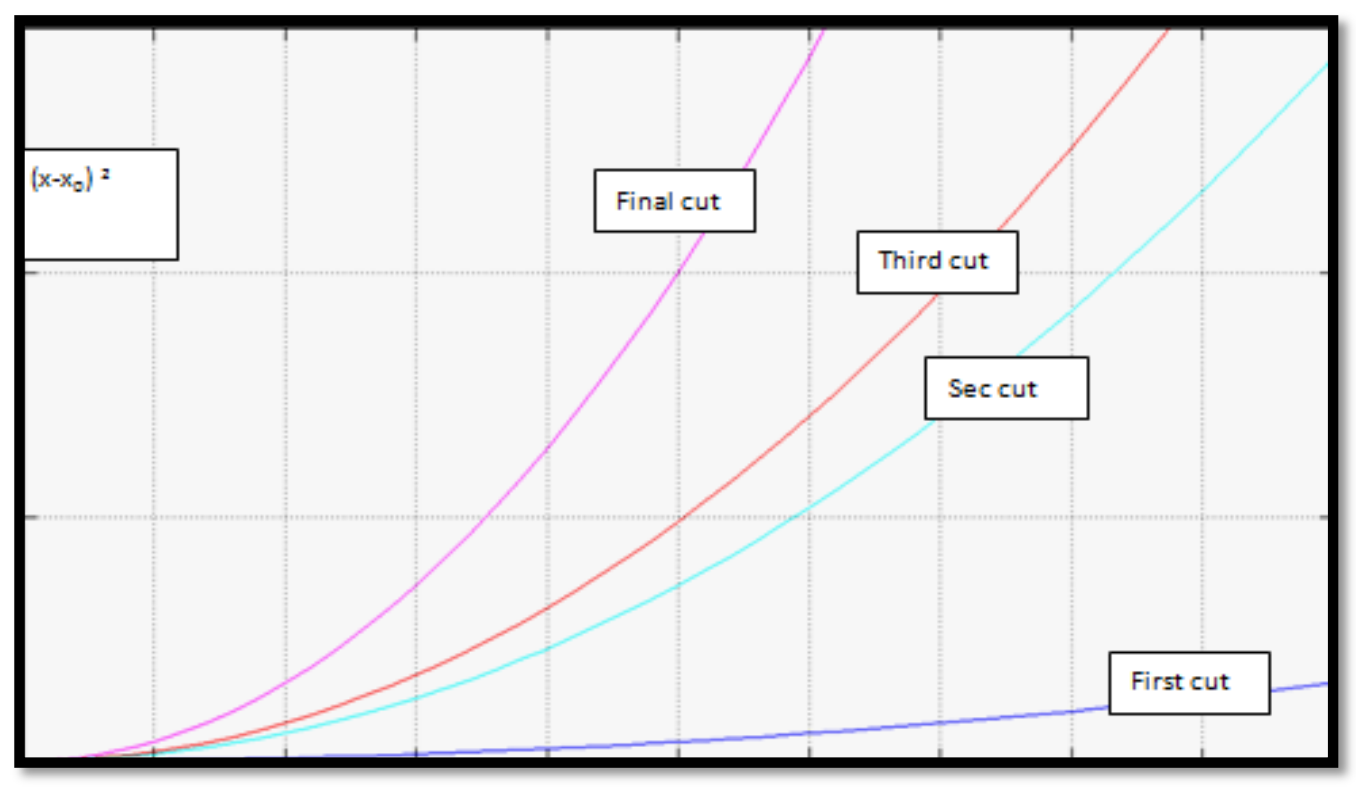

Figure 6.7 Simulation square of difference data using Simulink model

\subsubsection{Analyzing the Surface Response data}

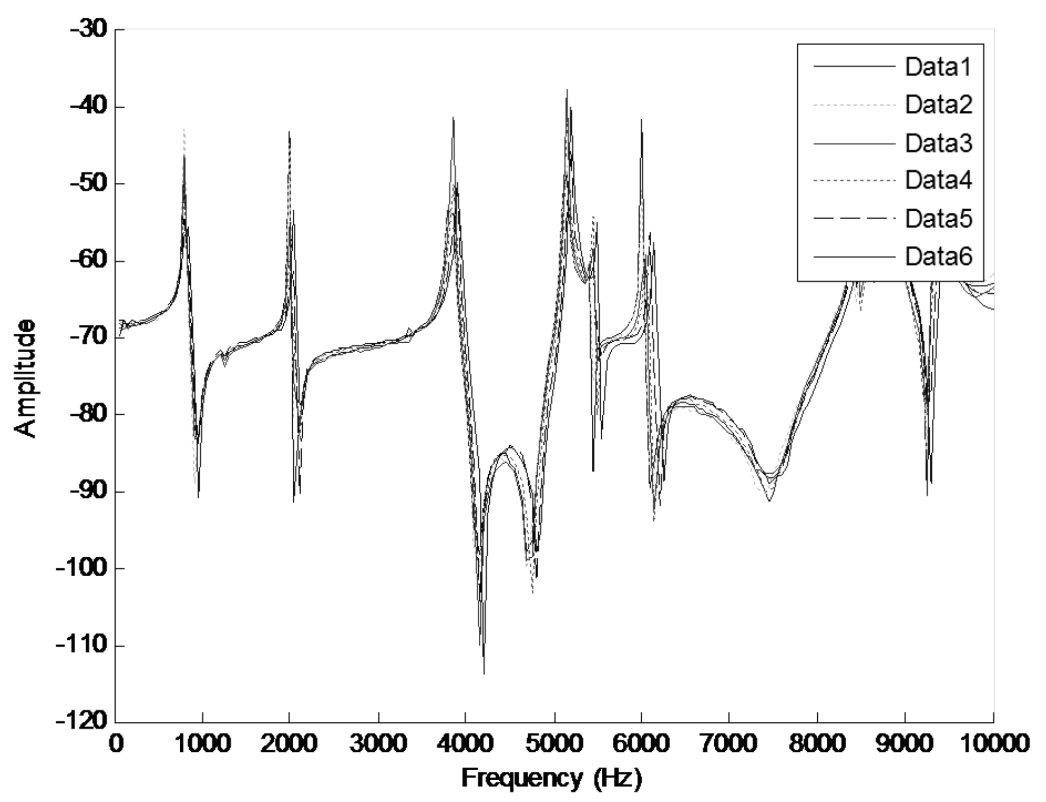

Figure 6.8 Frequency responses at the receiving sensor (a) Data1: No wear (b) Data 2Data 6: Slightly worn -extreme wear of the tool

The surface response was also recorded parallel with the Lamb wave data acquisition from a SR 780 signal analyzer. The frequency responses due to excitation at 
piezoelectric receiving actuator at different wear conditions were showed in the Figure 6.8. Datal to Data6 provides the signal data from no wear to complete wear in an increasing order of the end mill tool decay. The signal data showed could not describe the wear characteristics of the end mill tool. The difference among various stages of wear in completely analyzed using various signal processing techniques.

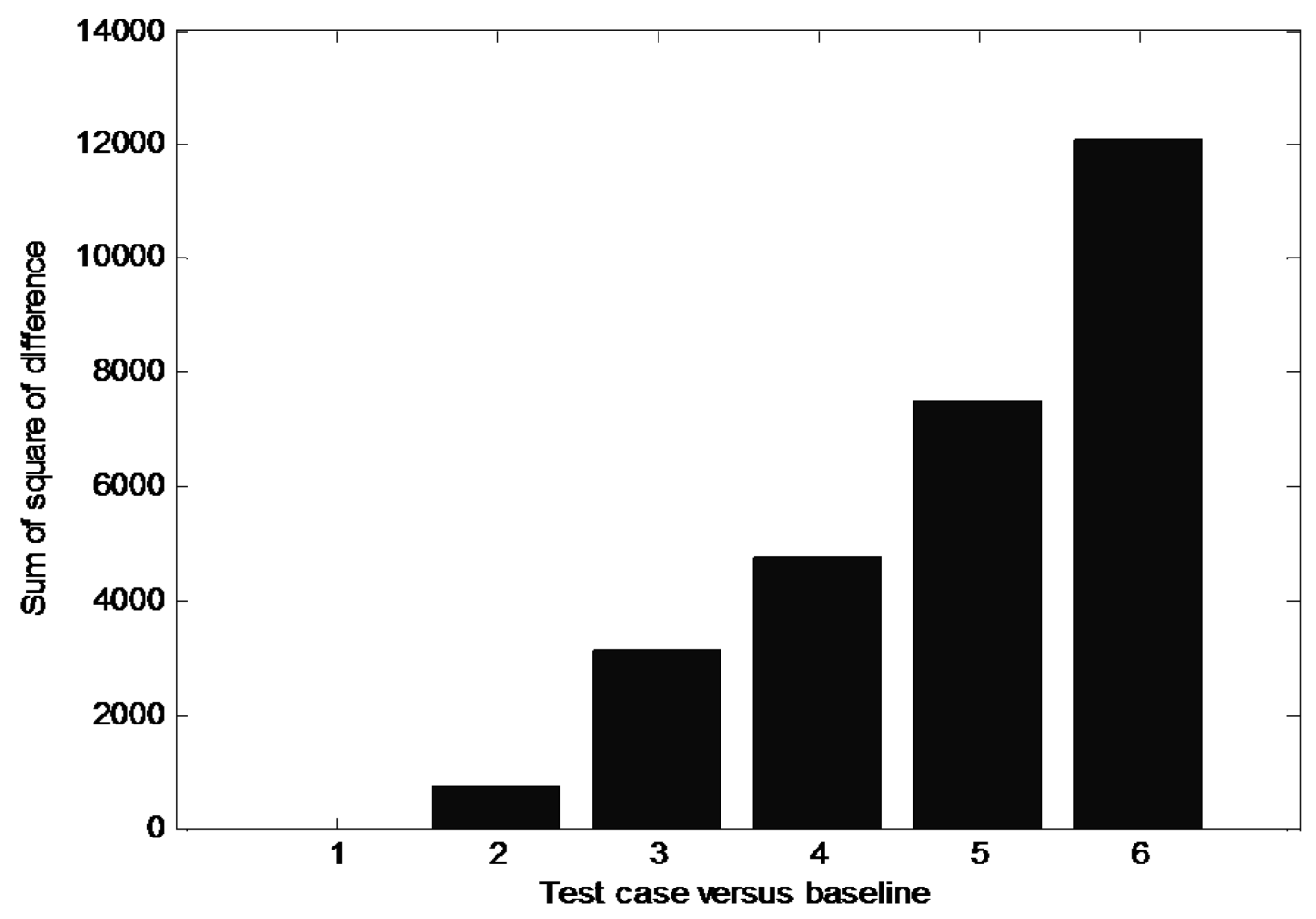

Figure 6.9 Comparison of sum square of difference among wear levels of the End mill 


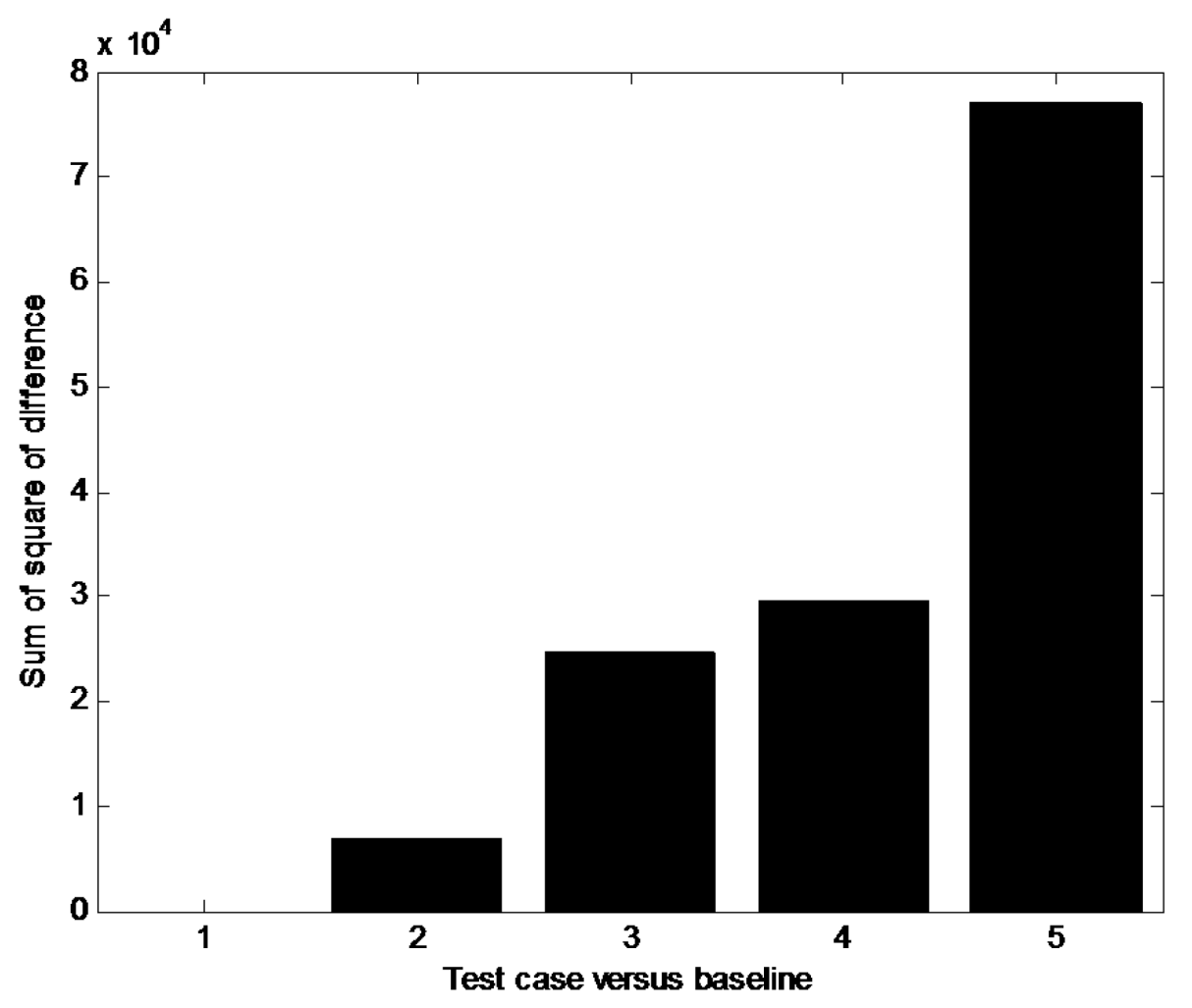

Figure 6.10 Comparison of sum square of difference among wear levels (Micro tool)

Sum of square of difference values were plotted using bar graphs in Figure 6.9. Each bar in the graph indicates a corresponding level of wear during the experiment. Test case 1 indicates no wear condition of the end mill, and the increase rate of artificially induced wear is apparently demonstrated in the other cases of the bar chart. Figure 6.10 indicate similar plot for the micro tool 
The bar representation of the sum square of difference data indicated the wear but it was hard to categorize the wear level. In order to overcome this disability the sum of the squares values were compared at dissimilar frequency intervals as described in Figure 6.11 and Figure 6.12 (Micro tool). The difference among the six test cases was studied in intervals starting with of $50 \mathrm{~Hz}$ frequency.

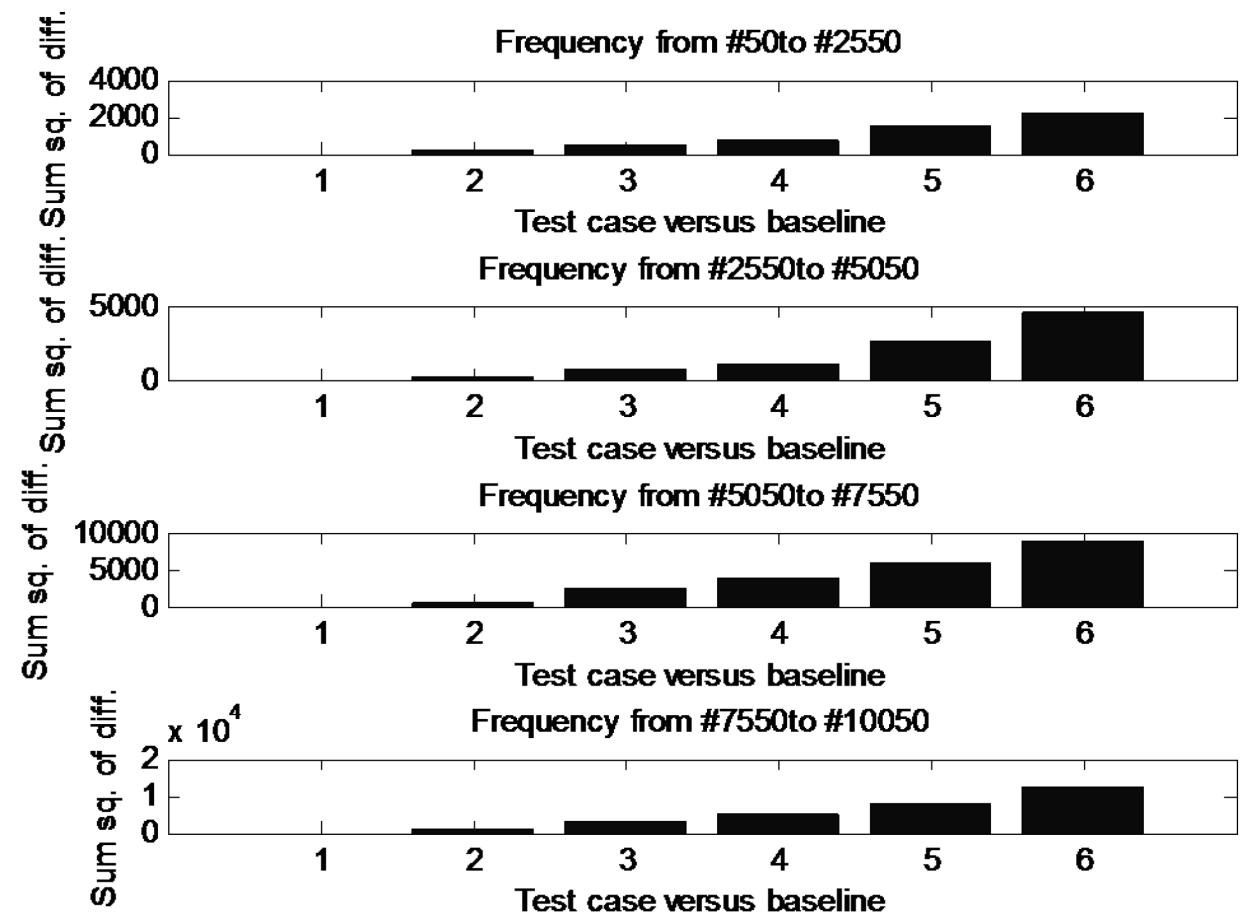

Figure 6.11 Comparison of sum square of difference (dissimilar frequency intervals) 


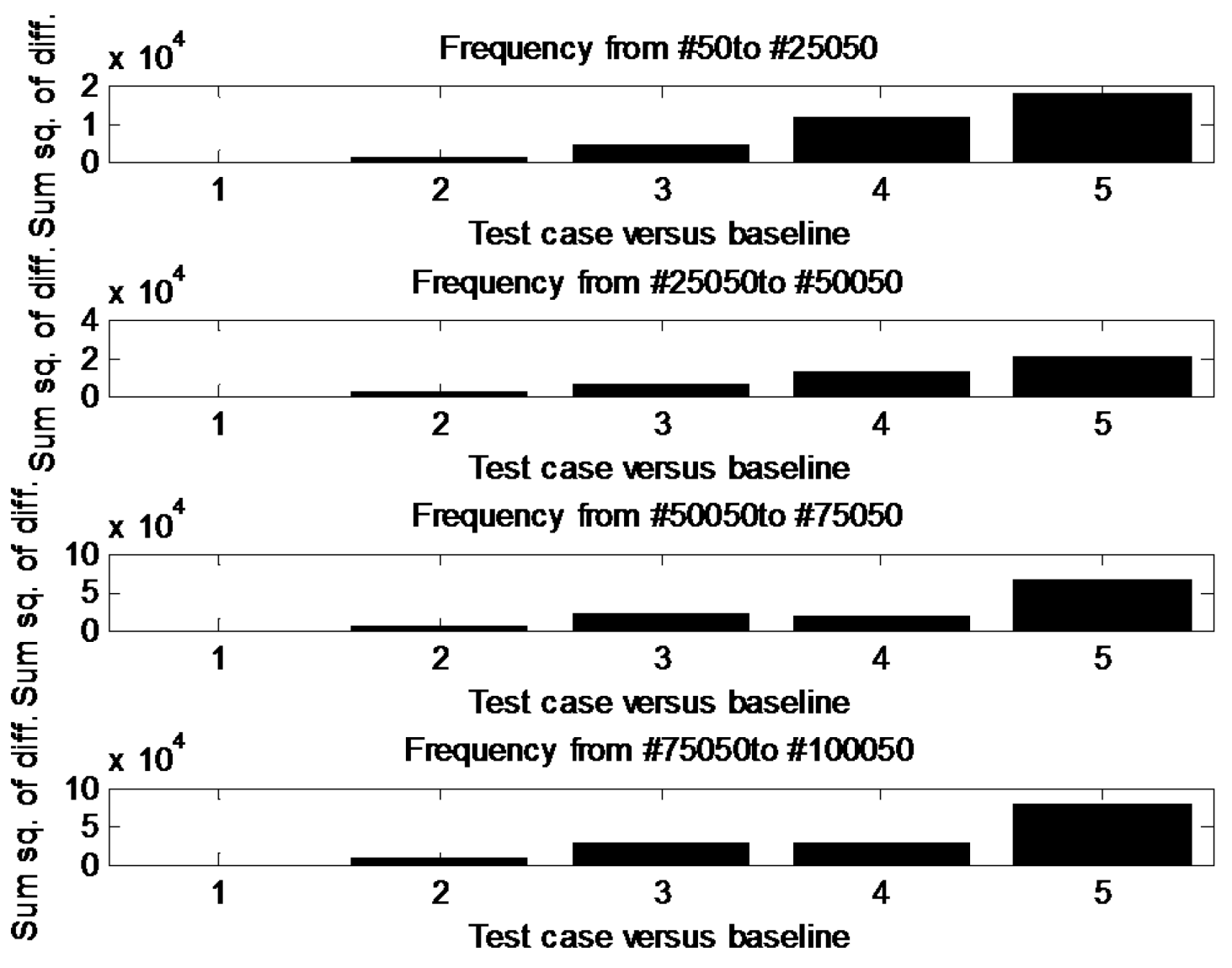

Figure 6.12 Comparison of sum square of difference for Micro tool (dissimilar frequency intervals)

The square of difference of the signal data was compared against frequency classified as slightly worn, medium wear, heavy wear severe wear and extreme wear. Sum square of difference at different bins of the mill tool are presented in Figure 6.13. The observation indicated that there was an incessant increase in the significant peak values of the sum square of differences. The difference in the peak values is able to separate the slightly worn tool from complete wear of the tip tool. 


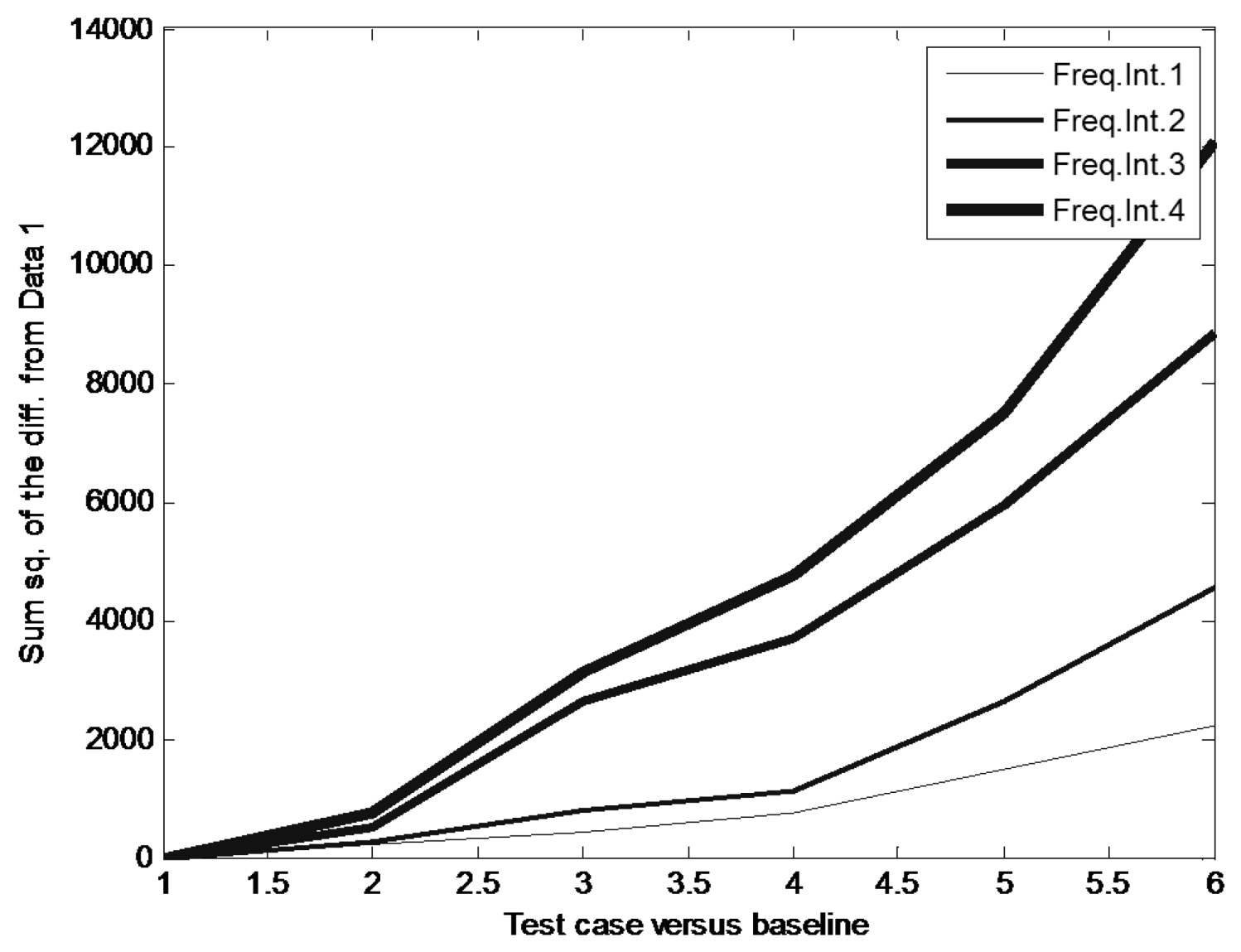

Figure 6.13 Comparison of the sum square differences at different bins

The peak values between significant data points are compared to investigate which significant peaks actually measuring the tool wear. Figure 6.14 details the significant peak information for the five stages of wear of the end mill. From the results it was observed that there are two significant peaks that are increasing form low level wear to the severe wear in ascending order. The first significant peak was observed at around $40^{\text {th }}$ data point ( $20.4 \mathrm{kHz}$ frequency) and the second significant peak was shown at $180^{\text {th }}$ point of the data ( $90 \mathrm{kHz}$ frequency). 


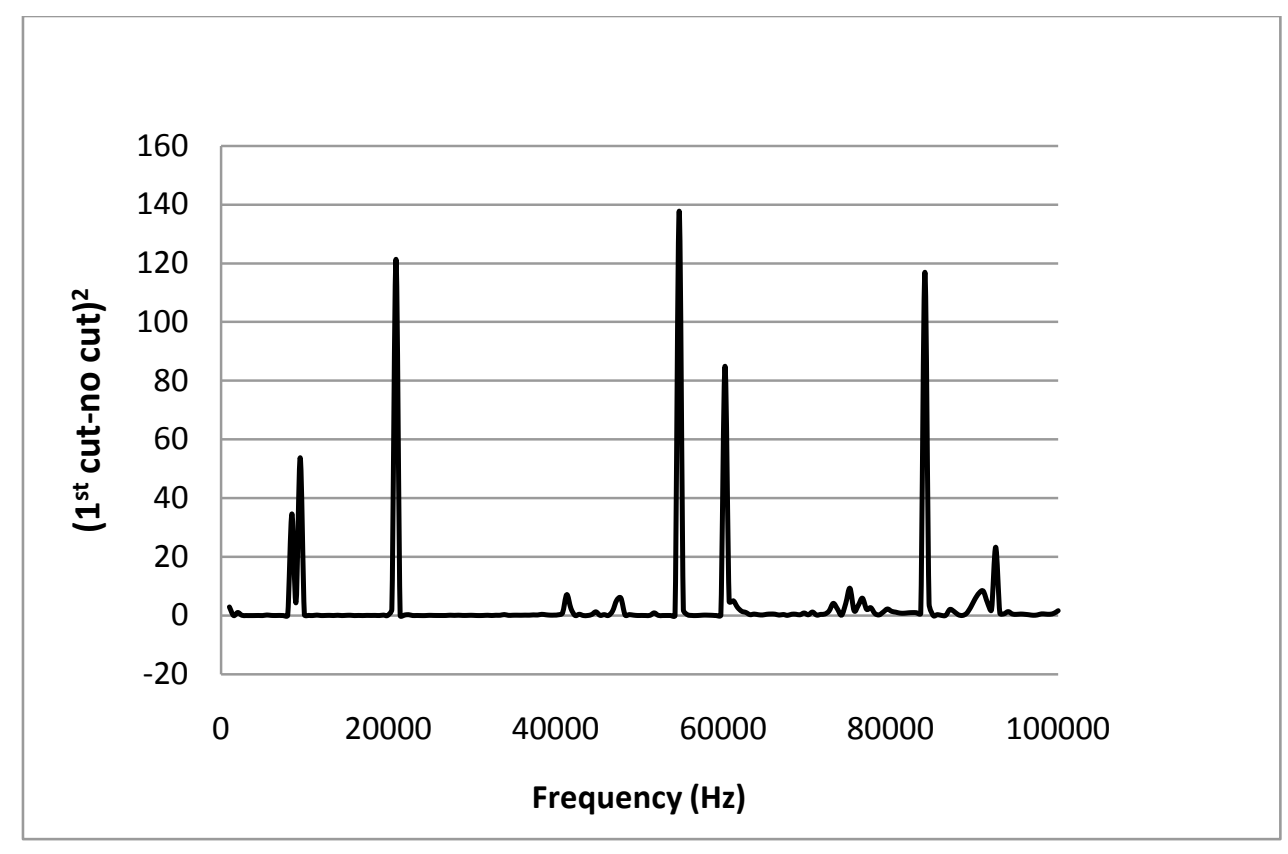

(a) No wear vs. Slightly worn

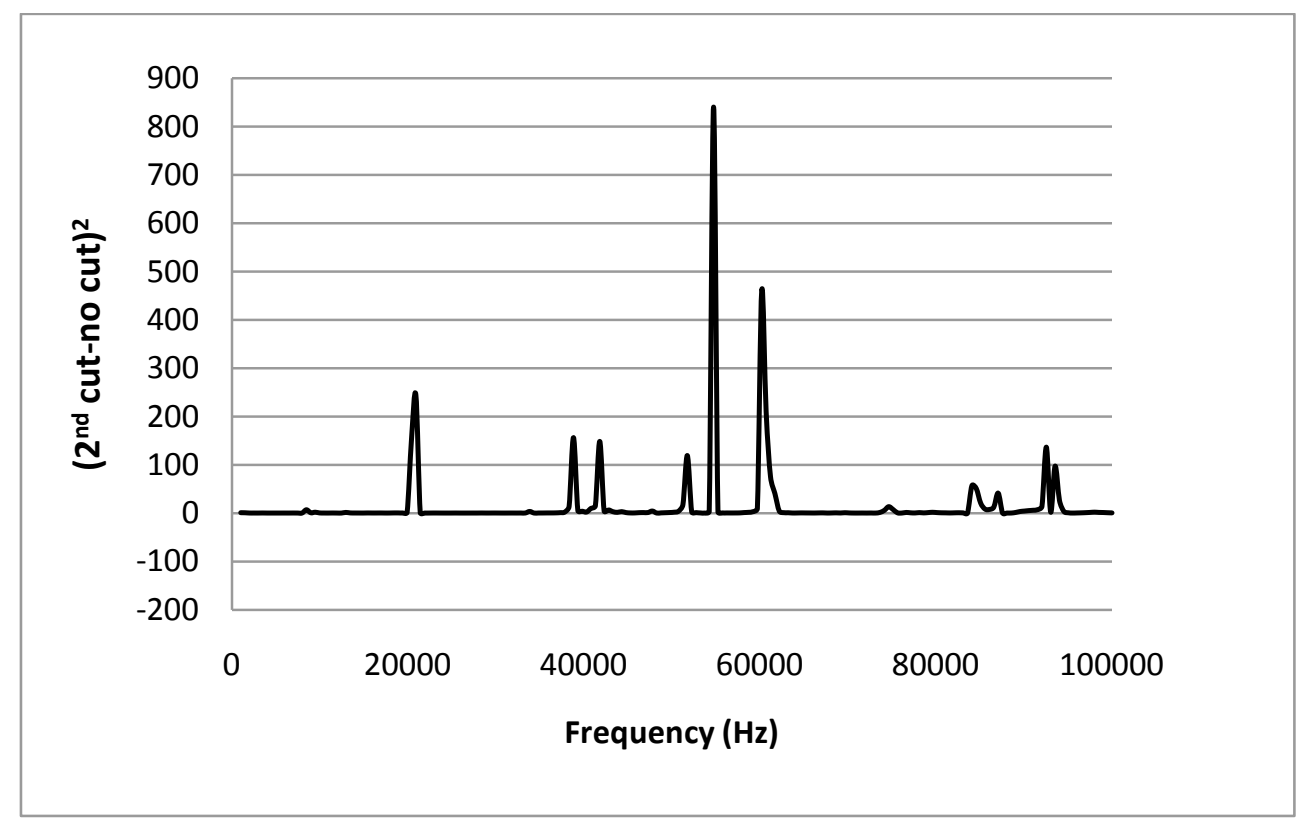

(b) No wear vs. Medium wear 


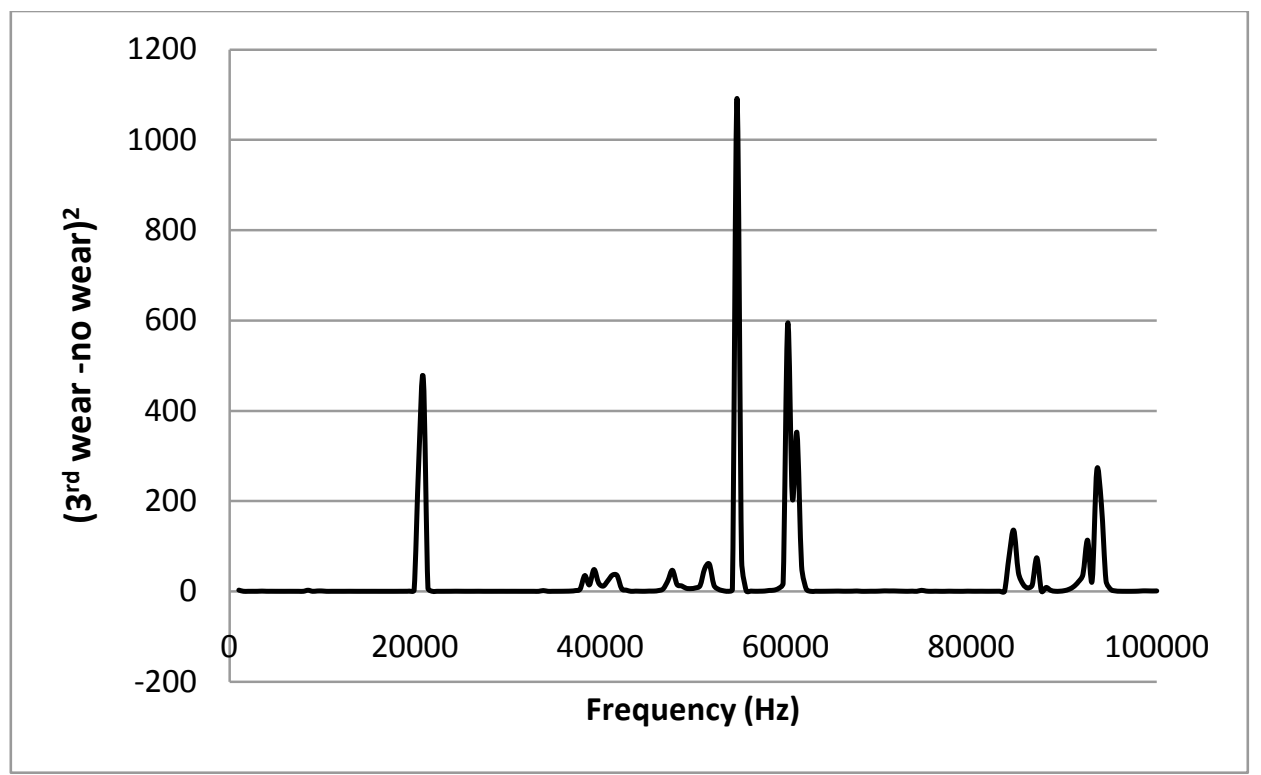

(c) No wear vs. Heavy wear

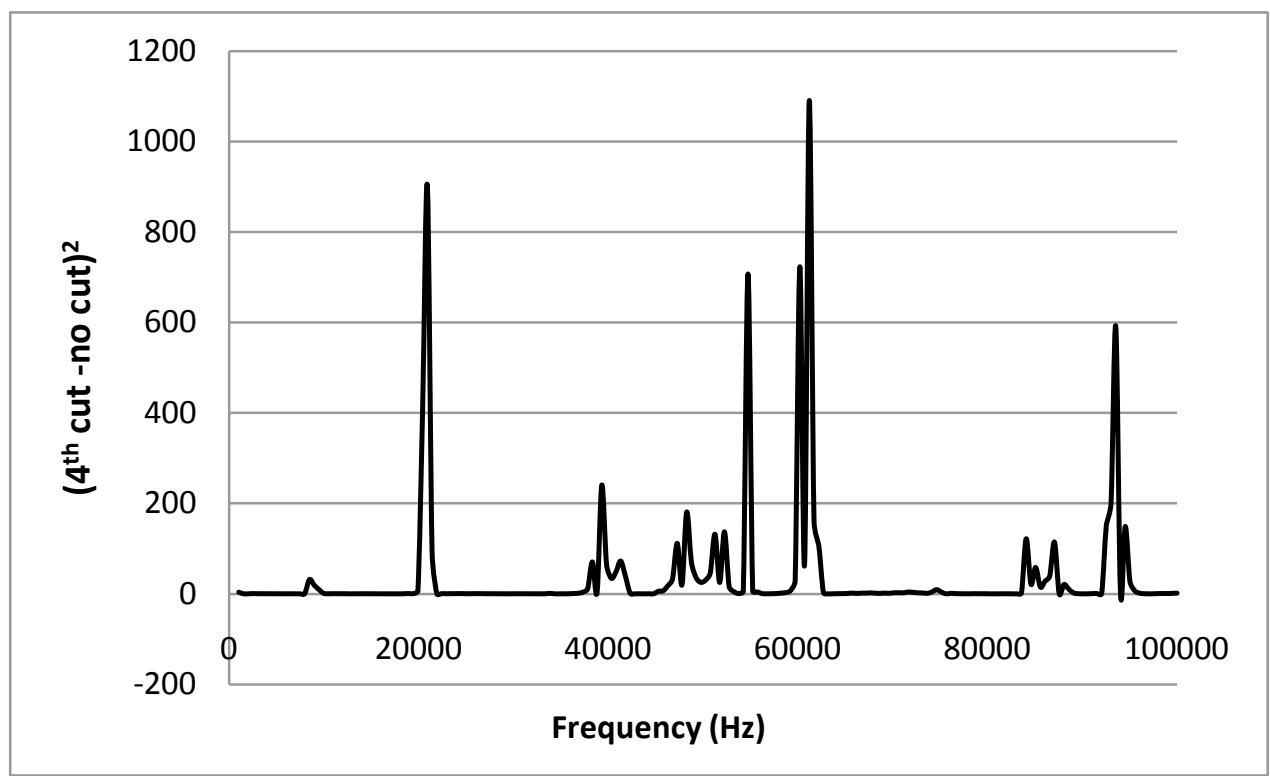

(d) No wear vs. Severe wear 


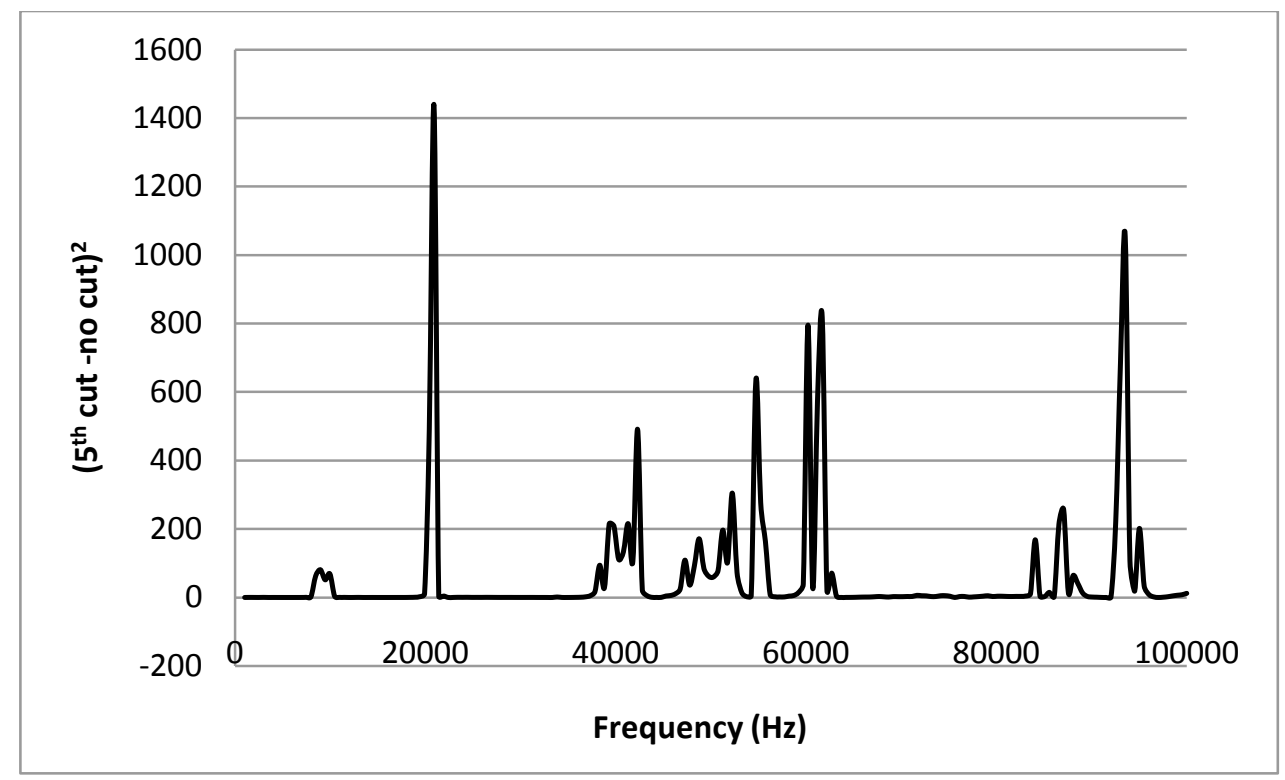

(e) No wear vs. Extreme wear

Figure 6.14 Comparison of peak data of the envelopes(a) No wear vs. Slightly worn (b) No wear vs. Medium wear (c) No wear vs. Heavy wear (d) No wear vs. Severe wear (e) No wear vs. Extreme wear

Table 6.1. List of square of difference peak values at $20.4 \mathrm{kHz}$ and $90 \mathrm{kHz}$ frequency

\begin{tabular}{|l|r|r|}
\hline Square of difference with no cut & $\begin{array}{l}\text { Peak value at 20.4 } \\
\mathrm{kHz}\end{array}$ & $\begin{array}{l}\text { Peak value at 90 } \\
\mathrm{kHz}\end{array}$ \\
\hline First wear (Low) & 121.41175 & 23.31007 \\
\hline Second wear (Medium) & 244.4526 & 136.5238 \\
\hline Third wear (Medium) & 470.668686 & 267.7504 \\
\hline Fourth wear (Severe) & 901.219012 & 590.3831 \\
\hline Fifth wear (severe) & 1434.61637 & 1055 \\
\hline
\end{tabular}




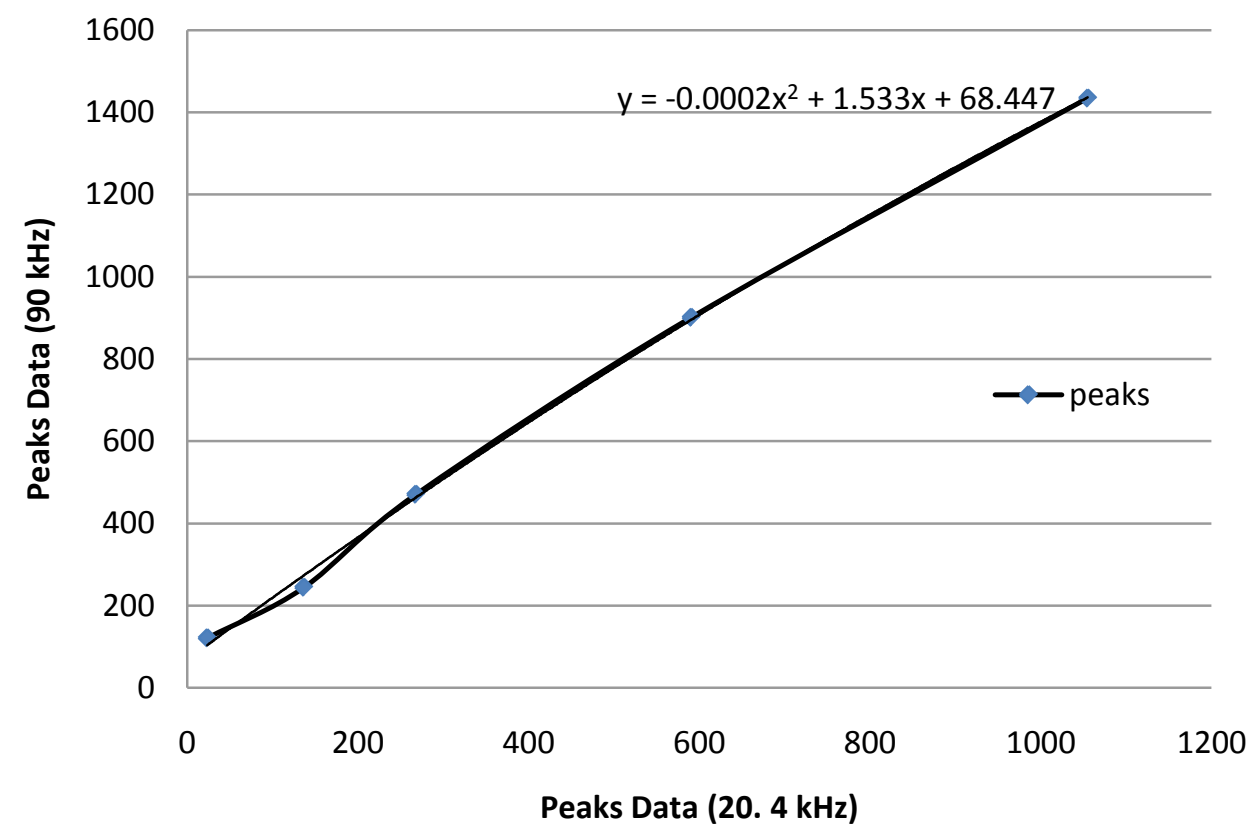

Figure 6.15 Comparison of peaks at $20.4 \mathrm{kHz}$ frequency and $90 \mathrm{kHz}$ frequency at different stage of wear

Figure 6.15 showed a consistent increase in the sum of square values of the peaks at $20.4 \mathrm{kHz}$ and $90 \mathrm{kHz}$ frequency. These significant peak values of these two cases were tabulated in Table 4.2. In order to determine the consistency, the wear levels at these two instances are compared in Figure 6.12.

\subsubsection{Binlet Analysis}

User defined bi-orthogonal filter (Srik_f7_H11) was included in MATLAB wavelet tool box as described in Chapter Four. Multi signal analysis in the wavelet tool box was used to analyze the frequency response signal data. The analysis was performed at different levels of wavelet decomposition. The level 1 coefficients obtained during the different stages of wear are compared in Figure 6. 16. The results showed an increase in 
the coefficient height from no wear to medium wear and diminished during the final stages of wear.

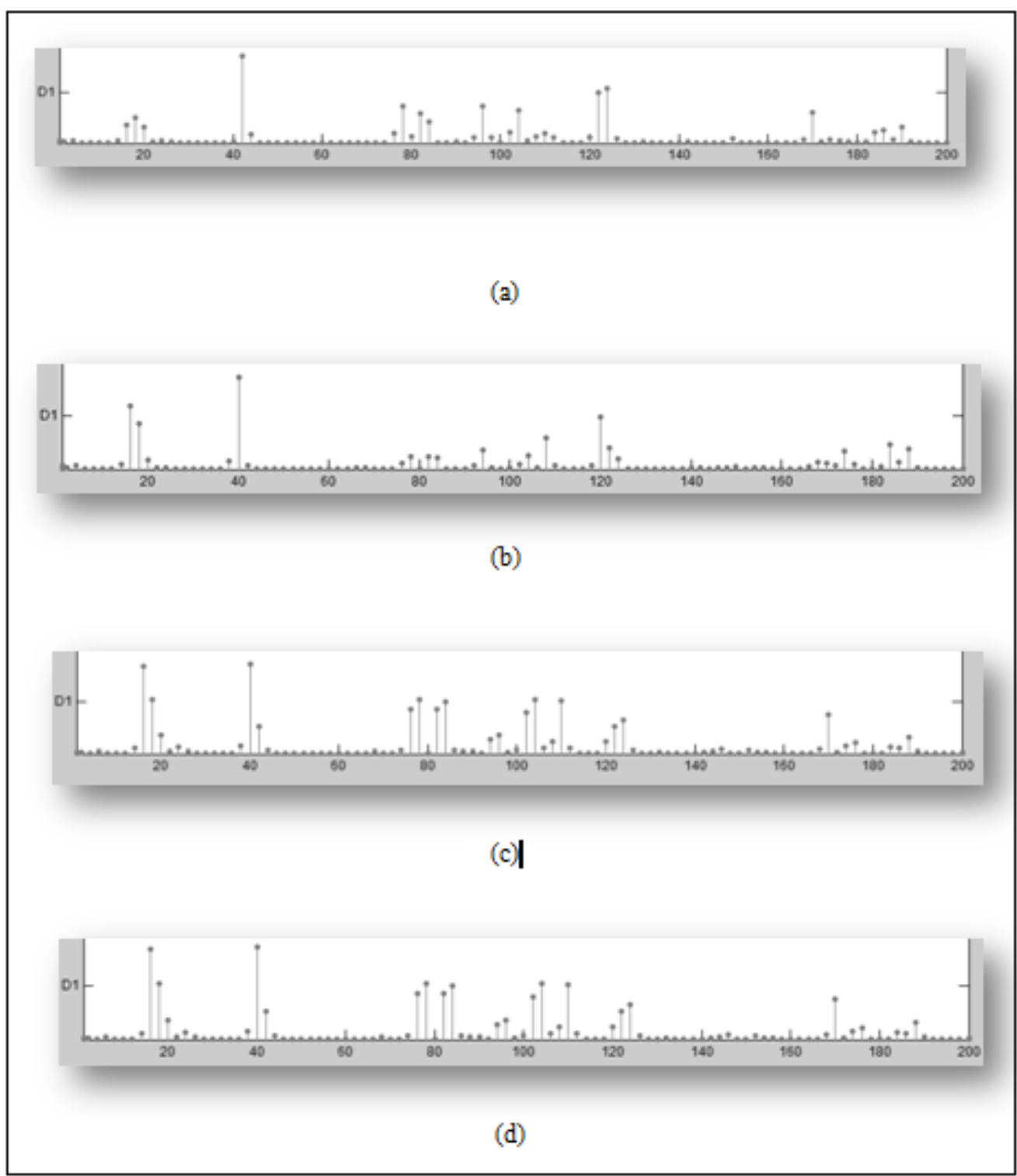

Figure 6.16 Binlet Coefficients: (a) no wear (b) slightly worn (c) medium wear (d) heavy wear 
The statistical residue coefficients of the signals were obtained from the binlet analysis and presented in Table 6.2. The standard deviation residue values were compared for all stages of wear in Figure 6.17, to understand the wear level. The graph showed a gradual increase in residue value, from no wear condition to the third stage of the wear. The standard deviation was lower during the severe wear condition of the tool. Mean absolute deviation against tool condition was also plotted in Figure 6.18.

Table 6.2 Statistical data at different wear levels

\begin{tabular}{|c|c|c|c|c|c|c|}
\hline & $\begin{array}{r}\text { No } \\
\text { wear }\end{array}$ & $\begin{array}{r}\text { Slightly } \\
\text { worn }\end{array}$ & $\begin{array}{r}\text { Medium } \\
\text { wear }\end{array}$ & $\begin{array}{c}\text { Heavy } \\
\text { wear }\end{array}$ & $\begin{array}{r}\text { Severe } \\
\text { wear }\end{array}$ & $\begin{array}{r}\text { Extreme } \\
\text { wear }\end{array}$ \\
\hline Mean & 0.0063 & 0.0061 & 0.0078 & 0.0078 & 0.0063 & 0.0066 \\
\hline Max & 1.0477 & 1.0472 & 1.4746 & 1.4746 & 1.0477 & 1.2223 \\
\hline Min & -0.9921 & -0.9982 & -1.1598 & -1.1598 & -0.9921 & -1.0727 \\
\hline Range & 2.0398 & 2.0454 & 2.6343 & 2.6343 & 2.0398 & 2.295 \\
\hline $\mathrm{SD}$ & 0.3645 & 0.3889 & 0.4054 & 0.4054 & 0.3645 & 0.3778 \\
\hline Median & 0.0307 & 0.0289 & 0.02555 & 0.0255 & 0.0307 & 0.0289 \\
\hline mode & 0.1366 & 0.0518 & -0.4397 & -0.4397 & 0.1366 & 0.136 \\
\hline $\begin{array}{l}\text { Mean abs } \\
\text { deviation }\end{array}$ & 0.278 & 0.3018 & 0.3035 & 0.3035 & 0.278 & 0.2911 \\
\hline $\begin{array}{r}\text { Median } \\
\text { abs } \\
\text { deviation }\end{array}$ & 0.2044 & 0.2316 & 0.2243 & 0.2243 & 0.2044 & 0.2206 \\
\hline
\end{tabular}




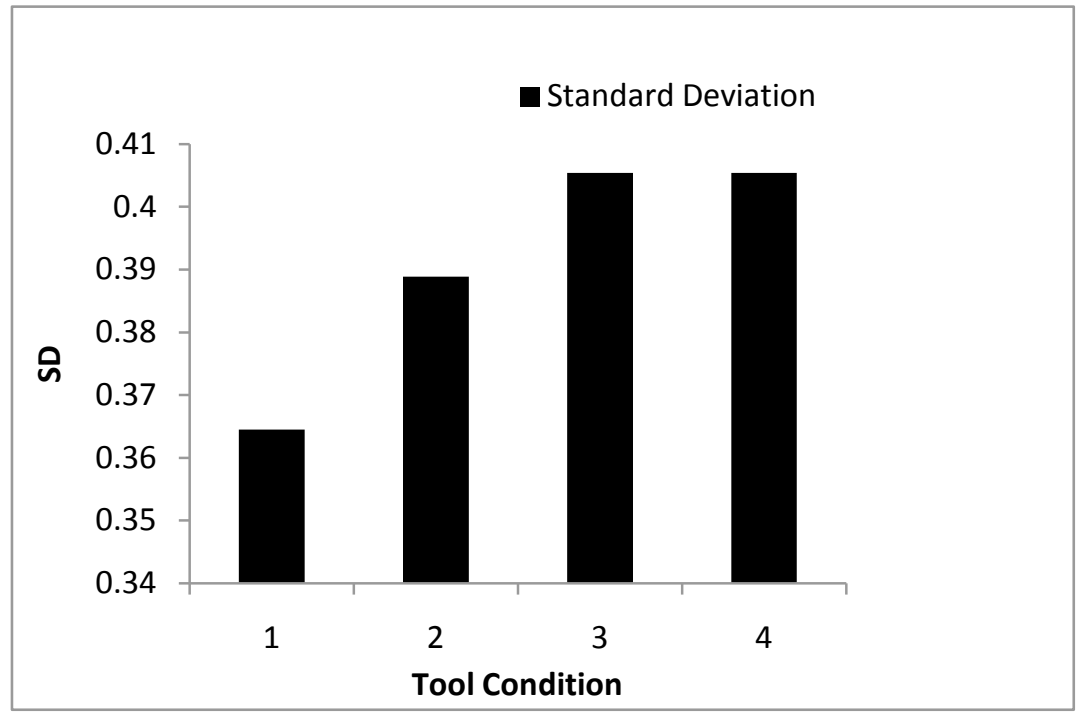

Figure 6.17 Comparison Residuals (SD): (a) No wear (b) Slightly worn (c) Medium wear (d) Heavy wear

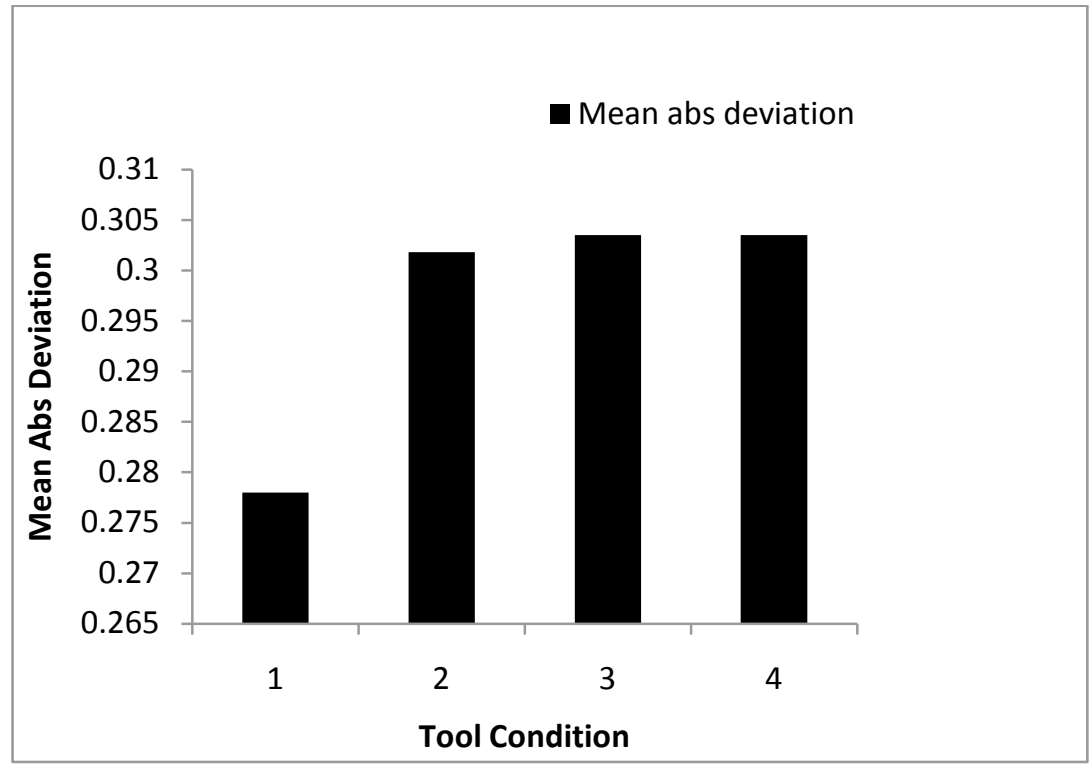

Figure 6.18 Comparison Residuals (Mean Abs. Deviation): (a) No wear (b) Slightly worn (c) Medium wear (d) Heavy wear 


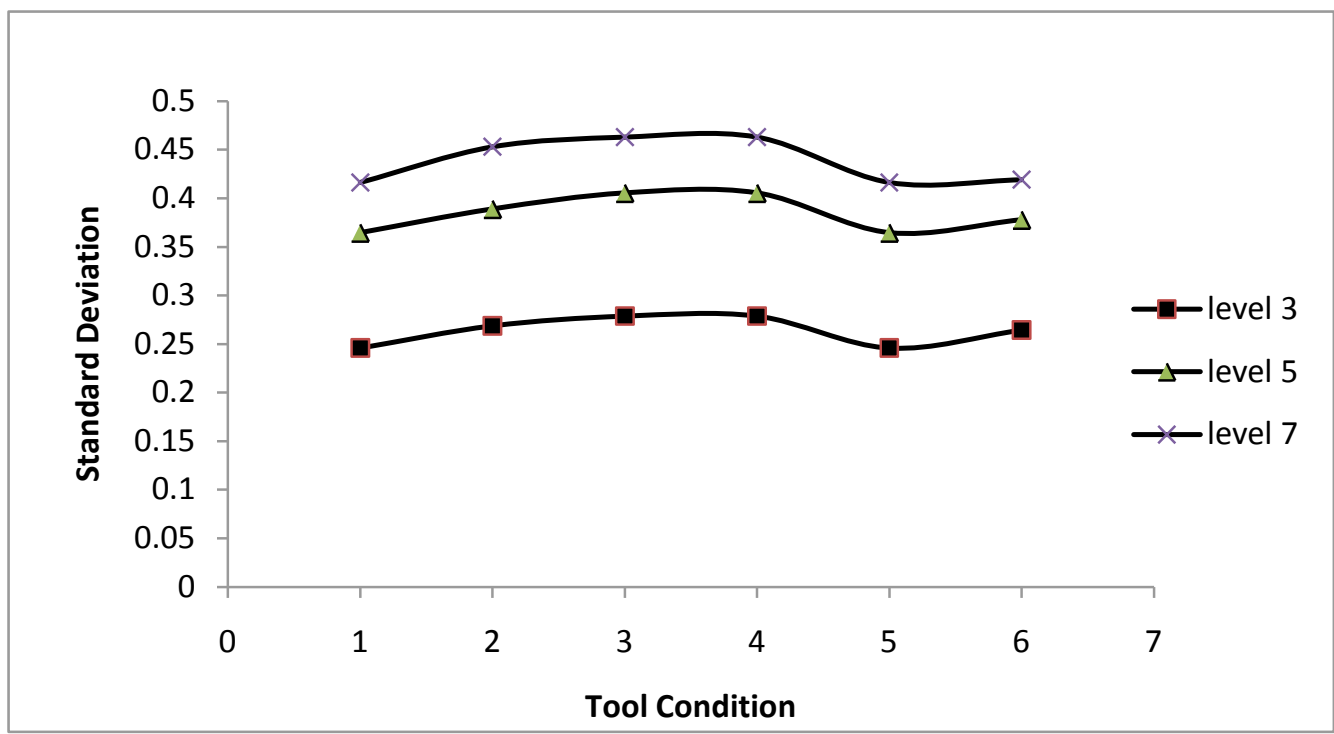

Figure 6.19 Comparison of SD residuals at three different levels of binlet analysis

The binlet analysis was performed to compare the statistical residue data during different levels of analysis (decomposition). Figure 6.19 compares the Standard deviation residuals during level 3, Level 5 and Level 7 stages of the analysis. All the plots showed a similar trend with different residue coefficients.

\subsection{Classification of Imperfections in Weld Joints}

The Lamb wave and SuRE techniques were used in this chapter to analyze a cut and hole damages on a weld joint. Binlet analysis was also performed to separate a cut from a hole. The amplitude and frequency envelopes using s-transformation primarily distinguish cut and a drilled hole of 0.0625 inch $(1 \mathrm{~mm})$ diameter on the butt weld.

\subsubsection{Lamb Wave Analysis}

The experiment was conducted in two steps Step 1: Initially, a cut of $1 / 4^{\text {th }}$ inch is created on the weld to record Lamb wave perturbations and surface response data. In Step 
2, a hole of 0.0625 inch diameter was drilled at the Centre of the butt weld. The Lamb wave signals of the imperfect welds when compared to the perfect weld signals were compared in Figure 6.20.
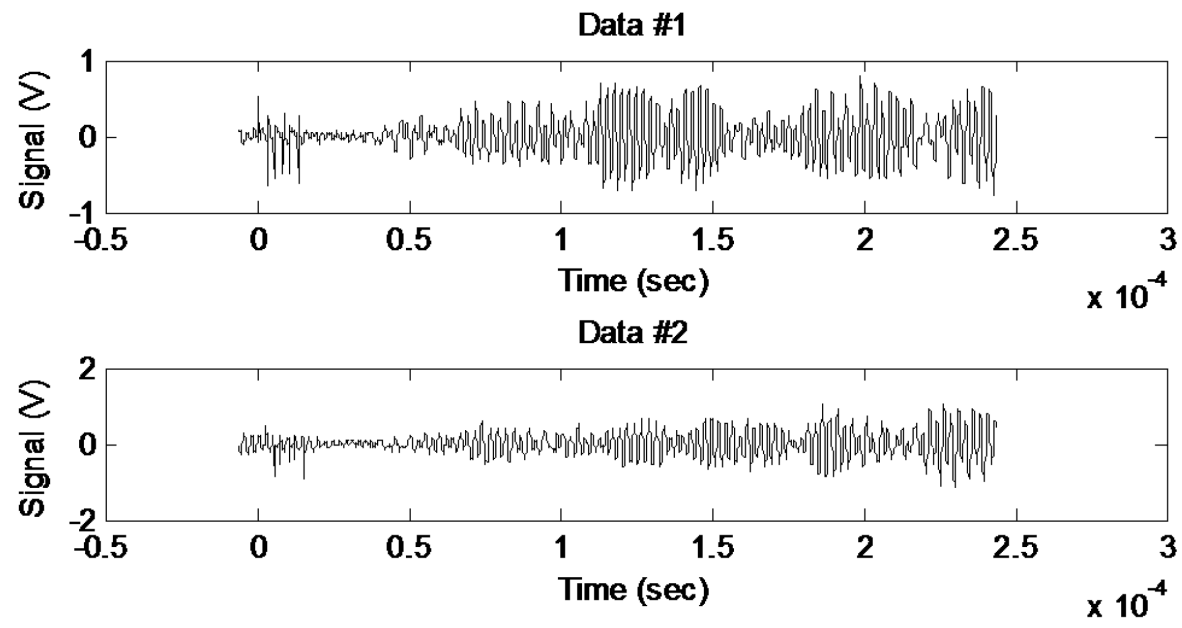

(a)
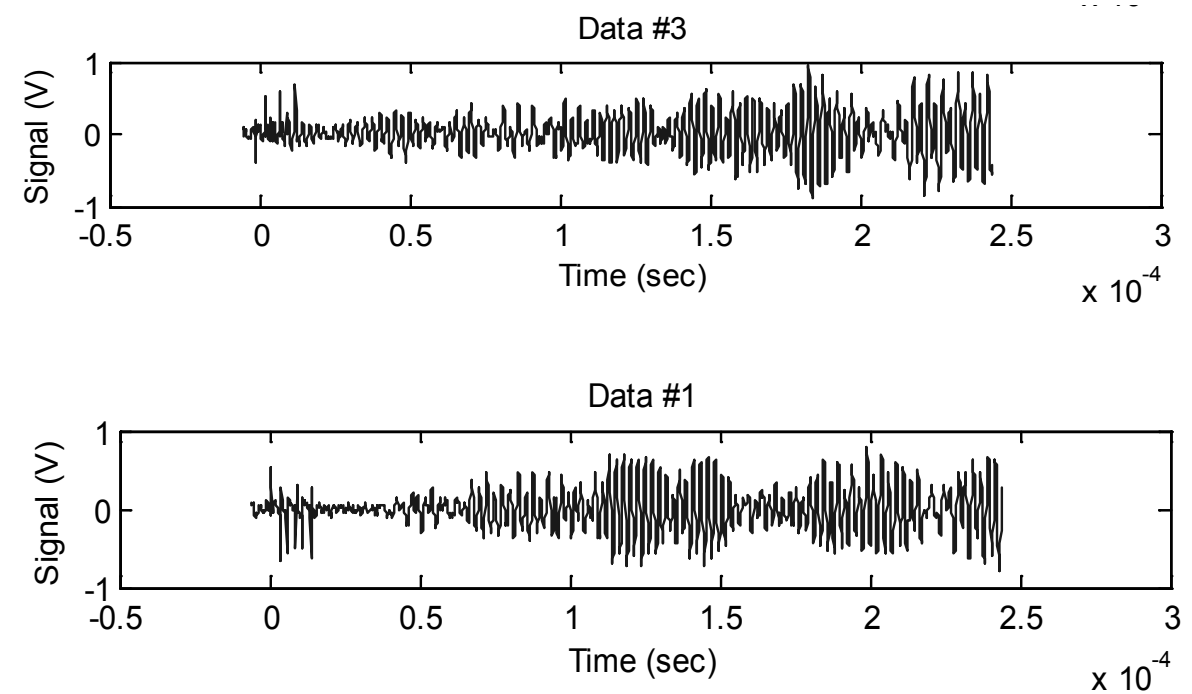

(b)

Figure 6.20 Comparison perfect signal data (Data 1) with signals with Imperfections (Data2): (a) $1 / 4^{\text {th }}$ inch cut (b) 0.0625 inch diameter hole (Lamb wave data) 


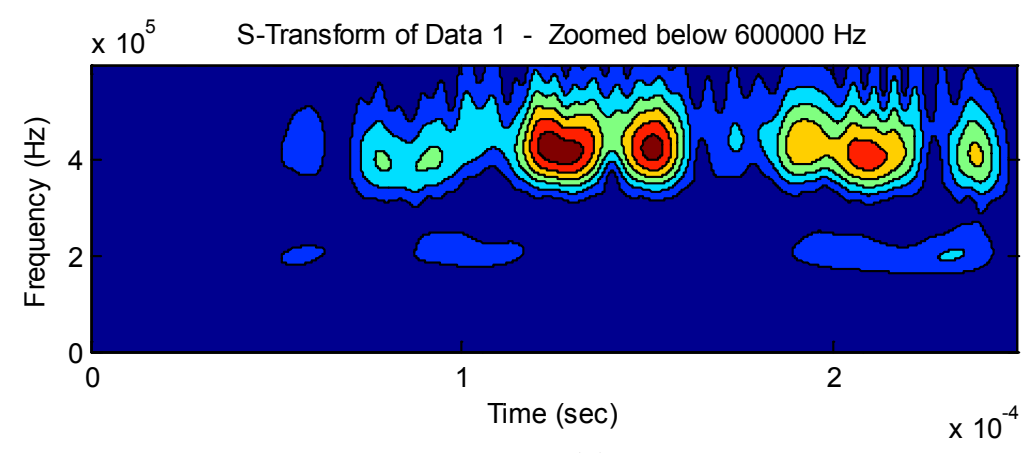

(a)

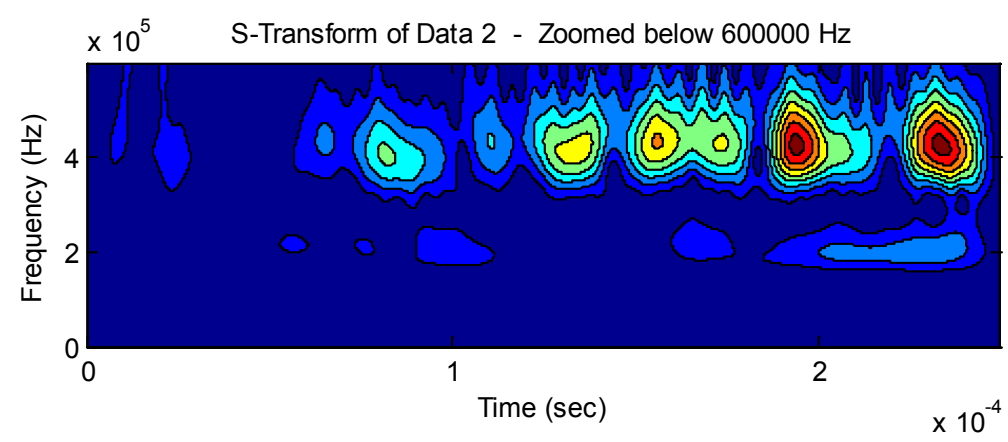

(b)

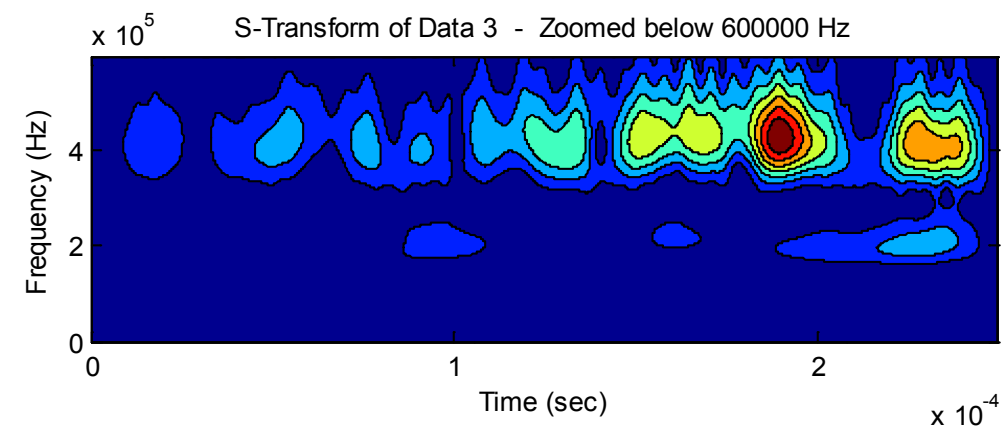

(c)

Figure 6.21 s-transformation data (Zoomed below $600000 \mathrm{~Hz}$ ) (a) Initial Signal (b) Cut (c) Hole

Comparison of the s-transformation (zoomed below $600000 \mathrm{~Hz}$ ) of the Lamb waves for a cut and a hole are showed in Figure 6.21 (b) and (c). The S transform of the 
perfect signal was showed in Figure 6.21(a). A change in s-transformation was observed among the perfect weld and the weld with a cut and hole.

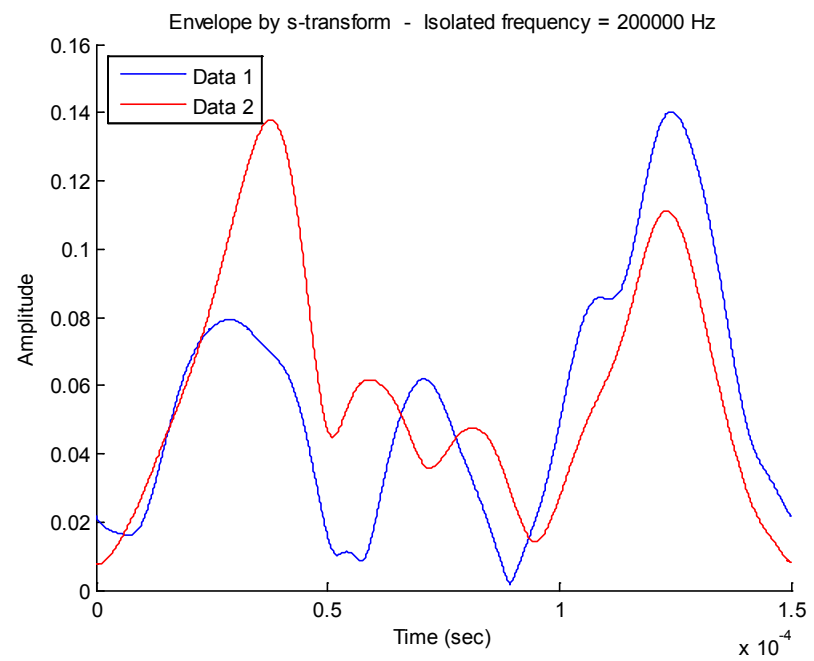

(a)

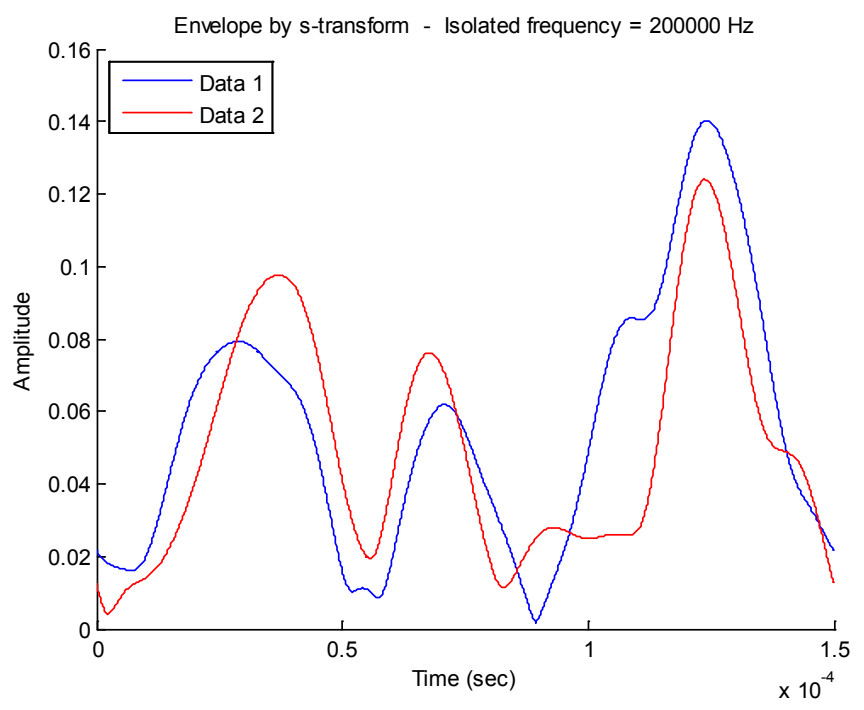

(b)

Figure 6.22 Comparison of s-transformation envelopes ( $200 \mathrm{kHz}$ ) between a (a) $1 / 4^{\text {th }}$ inch cut (b) 0.0625 inch diameter drilled hole 
A further analysis was made to compare the s-transformation envelopes of the imperfections with the perfect weld data. The Data 1 in Figure 6.22 (a), (b) represented the perfect weld data, while the Data 2 was the envelopes of the imperfection weld data. The $\mathrm{S}$ transform envelopes were taken at $200 \mathrm{kHz}$ frequency. It was observed that the peak of the S transform envelope was lowered in case of the drilled hole.

\subsubsection{Data Analysis with SuRE Approach}

The frequency response data for a $1 / 4^{\text {th }}$ inch cut and $1 \mathrm{~mm}$ diameter drilled hole of the weld were estimated as showed in Figure 6.23. The unprocessed Lamb wave data recorded as not able to distinguish a cut from the hole effectively. Therefore, Analysis was further conducted by determining the square of difference between Data1 and Data 2
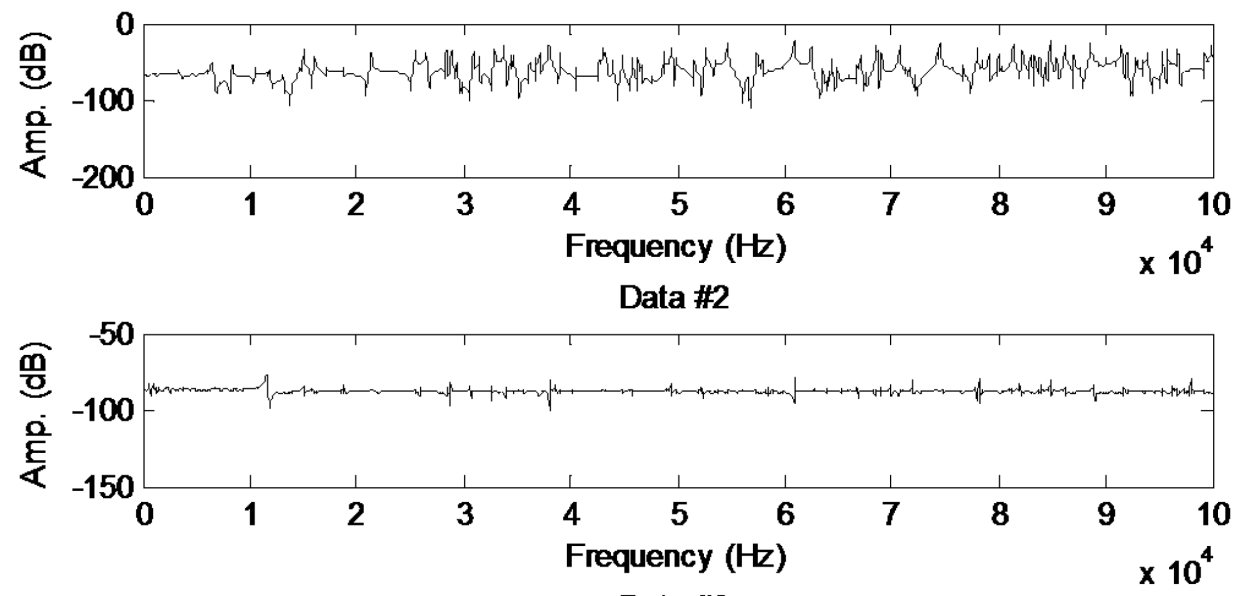

Data \#3

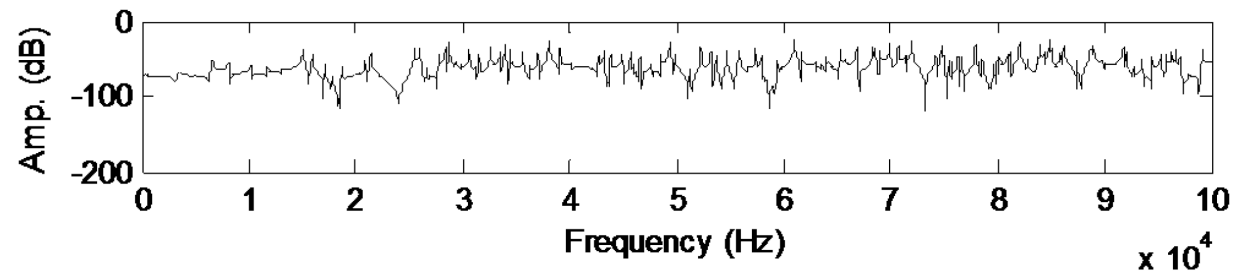

Figure 6.23 Comparison (a) perfect signal data (Data 1) with signals with Imperfections (Data2): (b) $1 / 4^{\text {th }}$ inch cut (c) 0.625 inch diameter hole (SuRE data) 
Figure 6.24 showed the difference of the amplitude levels between the perfect weld and the weld with imperfections. The amplitude of the difference was observed against the frequency. The Square of difference between the signal data with imperfections and the perfect weld are compared in Figure 6.25 Larger peaks were observed when the perfect weld was compared with the cut data. The amplitudes were smaller in case of the drilled hole.

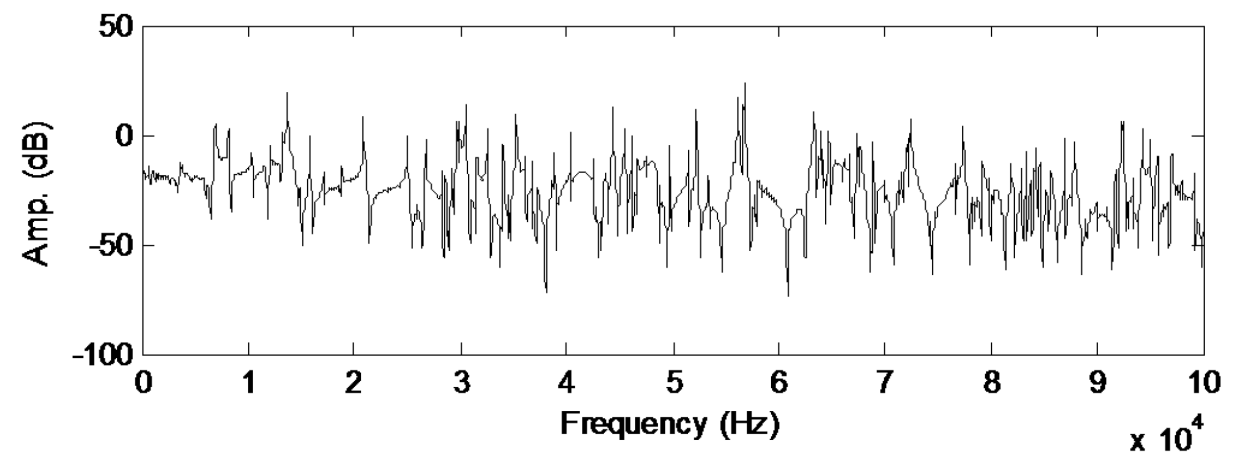

(a)

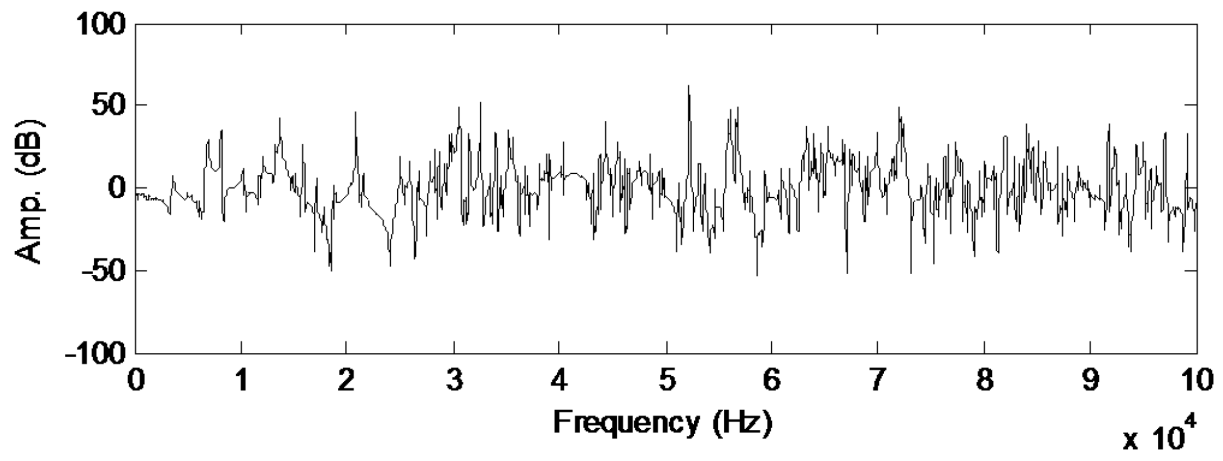

(b)

Figure 6.24 Comparison of amplitude difference between Perfect weld (data 1) and (a) Cut (b) Hole 

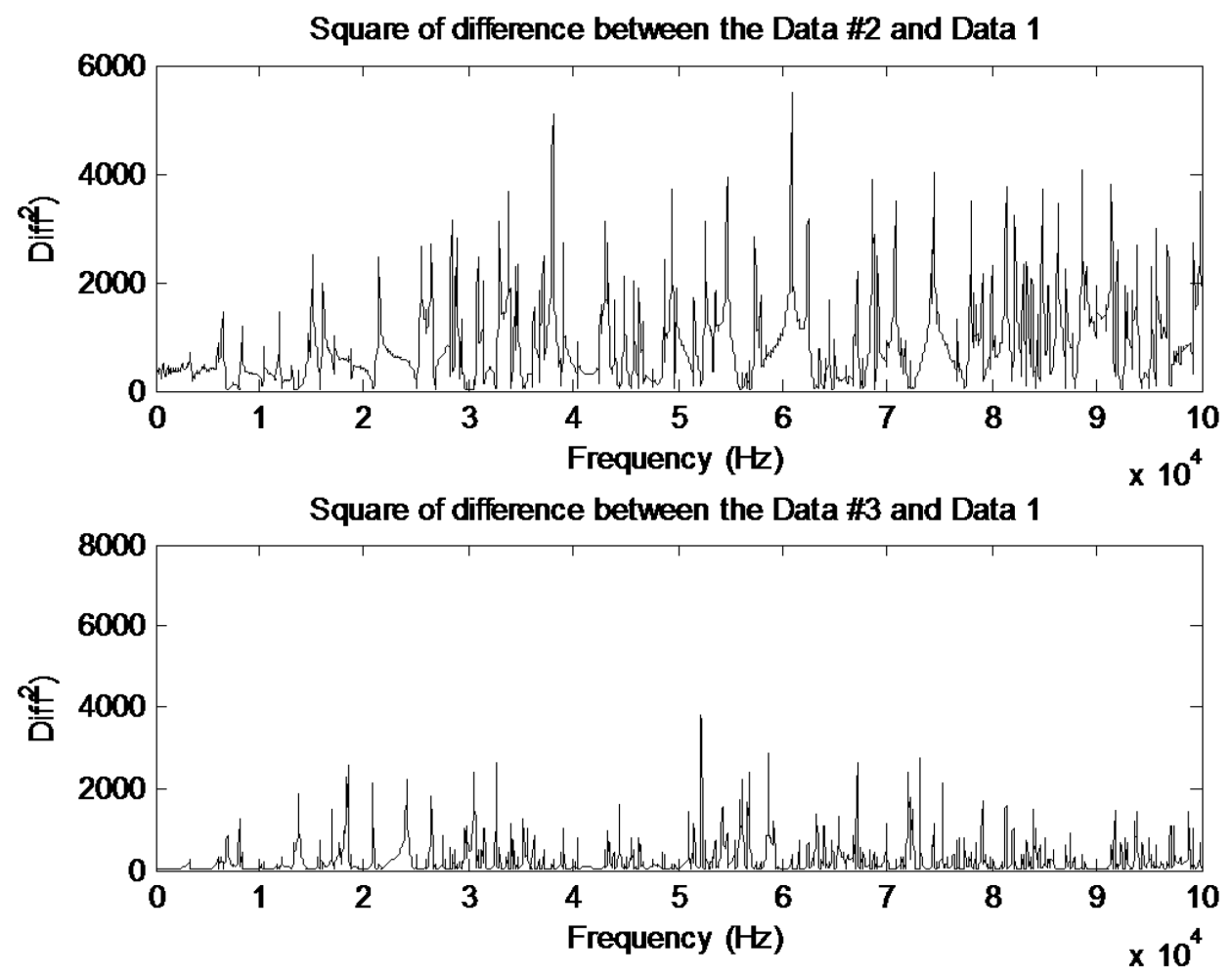

Figure 6.25 Comparison of square of difference between perfect weld (Data 1) and Cut (Data 2) and Hole (Data 3)

Sum of square of difference values were plotted using bar graphs in Figure 6.26. Each bar in the graph indicates the condition of the weld joint. Test case 1 indicates no the perfect weld. Test case 2 represents the sum square of difference in values due to the $1 / 4$ cut on the weld. Test case 3 Provide the information of the $1 \mathrm{~mm}$ drilled hole on the weld joint.

To compare the bar chart of the three cases of the weld joint, the same analysis is performed at dissimilar frequencies and presented in Figure 6.27. Comparison of sum of squares was accomplished in Figure 6.28 


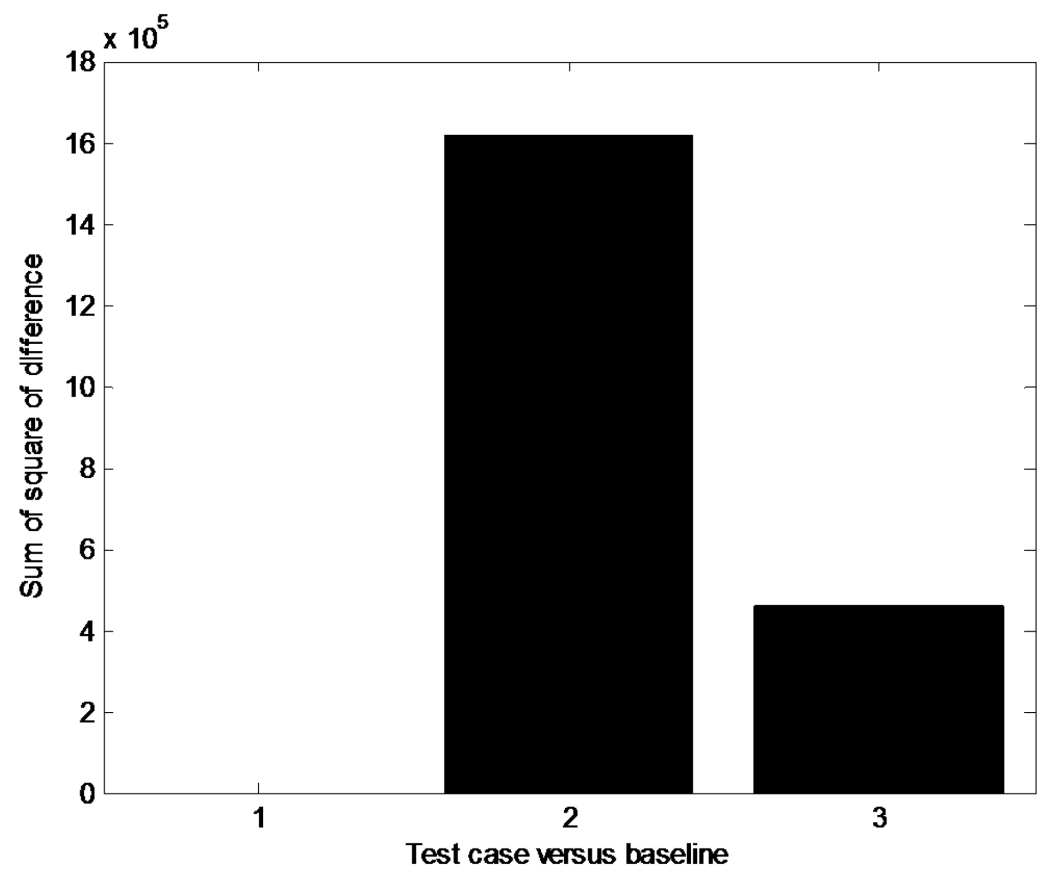

Figure 6.26 Comparison of sum square of difference between perfect and damaged data

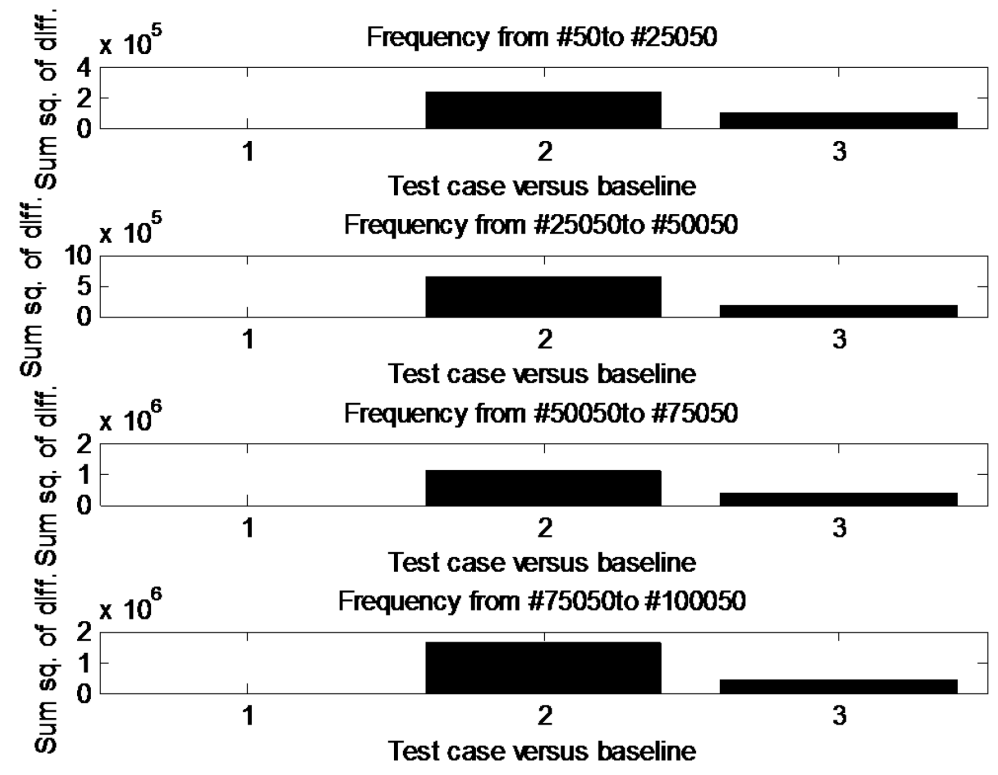

Figure 6.27 Comparison of sum square of difference between perfect and weld with Cut and Hole at dissimilar frequency intervals 


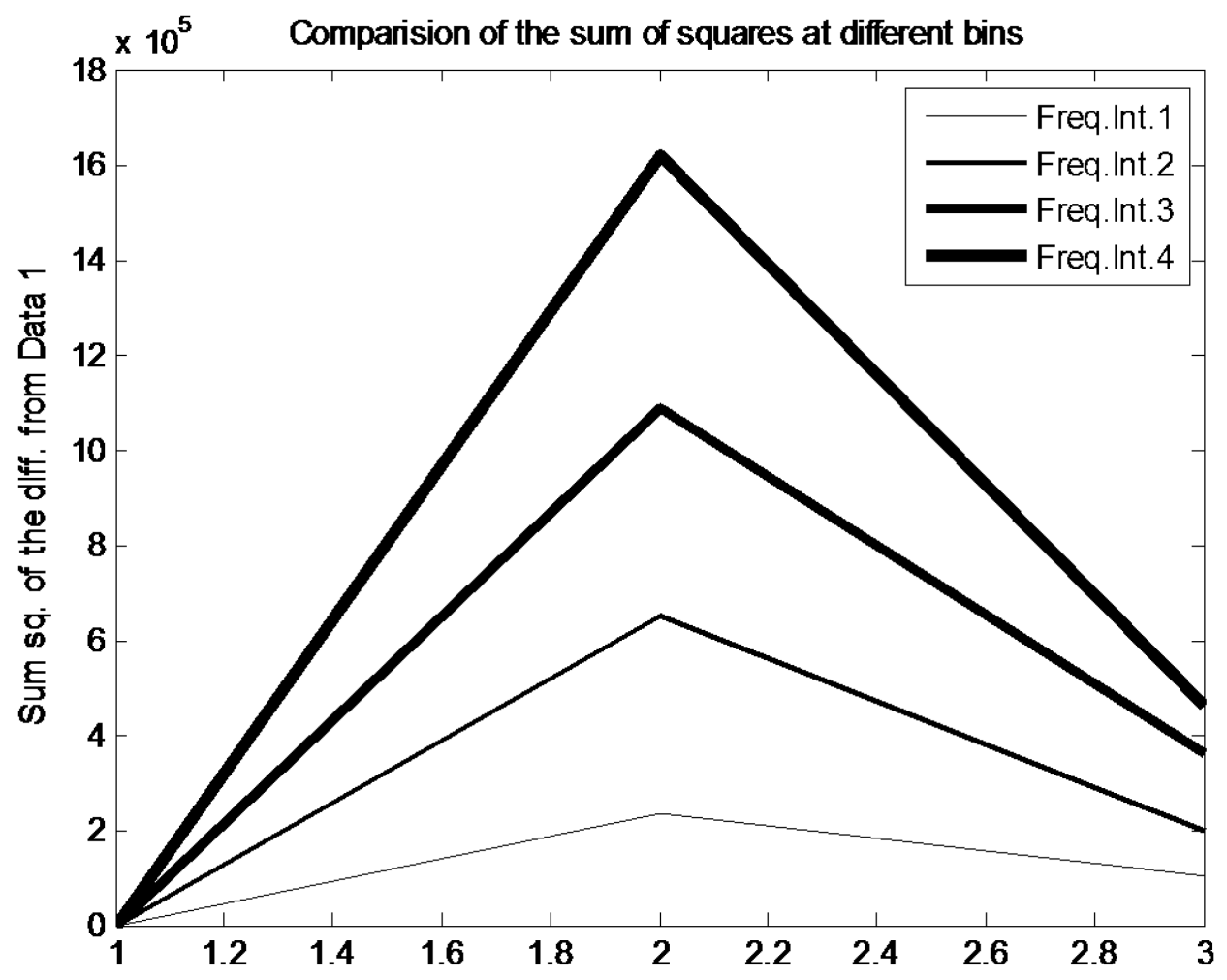

Figure 6.28 Comparison of Sum square of difference at different bins

The main idea that could differentiate various type of defects was to separate the shorter period oscillations in the signal from the longer oscillations and analyzing the local behavior of the signal. Initially the windowed fast Fourier transformation was identified to perform this kind of analysis. But the limitation is the window size and since the stage of the disturbance in the signal is unpredictable, this method of processing the data would be inconsistent. 


\subsubsection{Binlet Analysis}

The earlier results of the experiments were able to detect the defect at some level and the experimental results are significant. These results could not be able to distinguish the geometry of a defect. In the following sections, bi-orthogonal binary wavelets (Binlets) were used to effectively analyze the defects in structures. Recently MATLAB have been an essential tool for mechanical engineers as it is intended for numerical computing. MATLAB also engineers with other accessories like neural networks, fuzzy logics, control system and image processing tool boxes during optimization and signal processing. The wavelet tool box is also one of such important tool in MATLAB for signal and image processing. Using wavelet tool box one can perform various kinds of analysis using the software defined filters. It facilitates to execute 1-D signal processing and 2-D image processing very effectively.

Signal processing can be conducted using continuous and discrete wavelet transforms. Wavelet tool box also facilitates single signal and multi signal analysis. In the following sections of this chapter the results of multiple 1-D are used to compare the damages in various structures and cutting tools. The first level decomposition coefficients obtained during the multi scale wavelet analysis of the wavelet tool box environment were compared between a cut and drilled hole as showed in Figure 6.29 (a), (b).

The statistical data was taken by further analyzing the decomposition coefficients. It was observed that the density of the coefficients was increased during the first data points in case of the drilled hole. The height of the coefficients was more in case of a cut and a distributed behavior of the coefficients was observed. In order to distinctly separate 
the hole form the cut a statistical analysis was made using the residue coefficients obtained during binlet analysis.

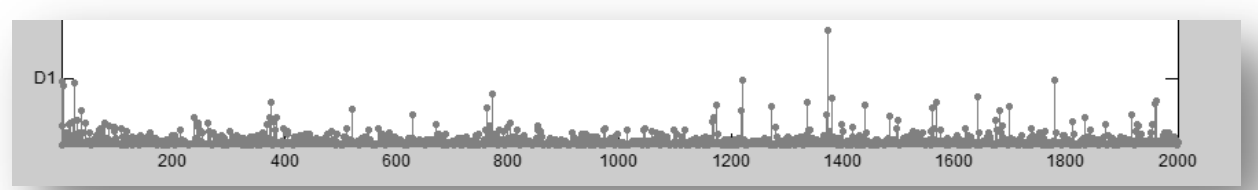

(a)

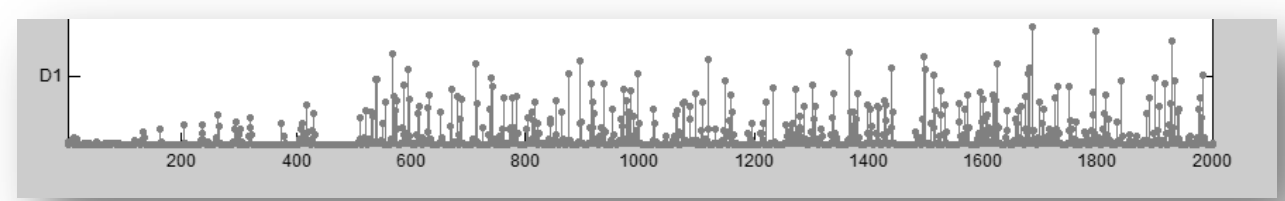

(b)

Figure 6.29 Binlet coefficients (a) $1 / 4$ inch cut (b) 0.0625 inch diameter drilled hole (D1 level decomposition)

The residue statistical data (mean, mode, SD etc.) was obtained from the binlet coefficients discussed in the previous section. The residue coefficient data was evaluated and used to distinguish dissimilar imperfections in the weld joints successfully. The statistical data obtained during the residue analysis was compared with the original weld data and listed in Table 4. 
Table 6.3 Comparison of statistical data

\begin{tabular}{|l|r|r|r|r|r|r|}
\hline & Trail 1 & Trail 2 & Trail 1 & Trail 2 & Trail 1 & Trail2 \\
\hline mean & -0.0013 & -0.002 & 0.0017 & -0.0012 & -0.0015 & 0.0016 \\
\hline max & 1.5721 & 1.633 & 0.9825 & 0.4677 & 1.483 & 1.4753 \\
\hline min & -1.4402 & -2.0835 & -2.3393 & -0.3888 & -1.6841 & -2.3607 \\
\hline range & 3.0123 & 3.7165 & 3.3218 & 0.8565 & 3.167 & 3.8359 \\
\hline SD & 0.3969 & 0.4216 & 0.0847 & 0.0703 & 0.4114 & 0.4187 \\
\hline Median & -0.0415 & -0.004 & -0.0008 & -0.0005 & 0.0053 & 0.0049 \\
\hline Mode & -0.0947 & 0.0225 & -0.014 & 0.0052 & 0.0261 & 0.0176 \\
\hline $\begin{array}{l}\text { Mean abs } \\
\text { dev }\end{array}$ & 0.2909 & 0.325 & 0.0345 & 0.0295 & 0.3046 & 0.3109 \\
\hline
\end{tabular}

A detailed analysis was made by comparing the perfect and defective residue statistical data in Figure 6.23. This graph apparently separated a hole from a cut. The percentage deviation of the peak values of the statistical data was compared to measure the variation.

The standard deviation (SD) and mean values were compared in the Figure 6.30 to determine the characteristics of the drilled hole. Higher deviations in the mean residue coefficients were observed during a cut, and comparatively the values low for the hole. 


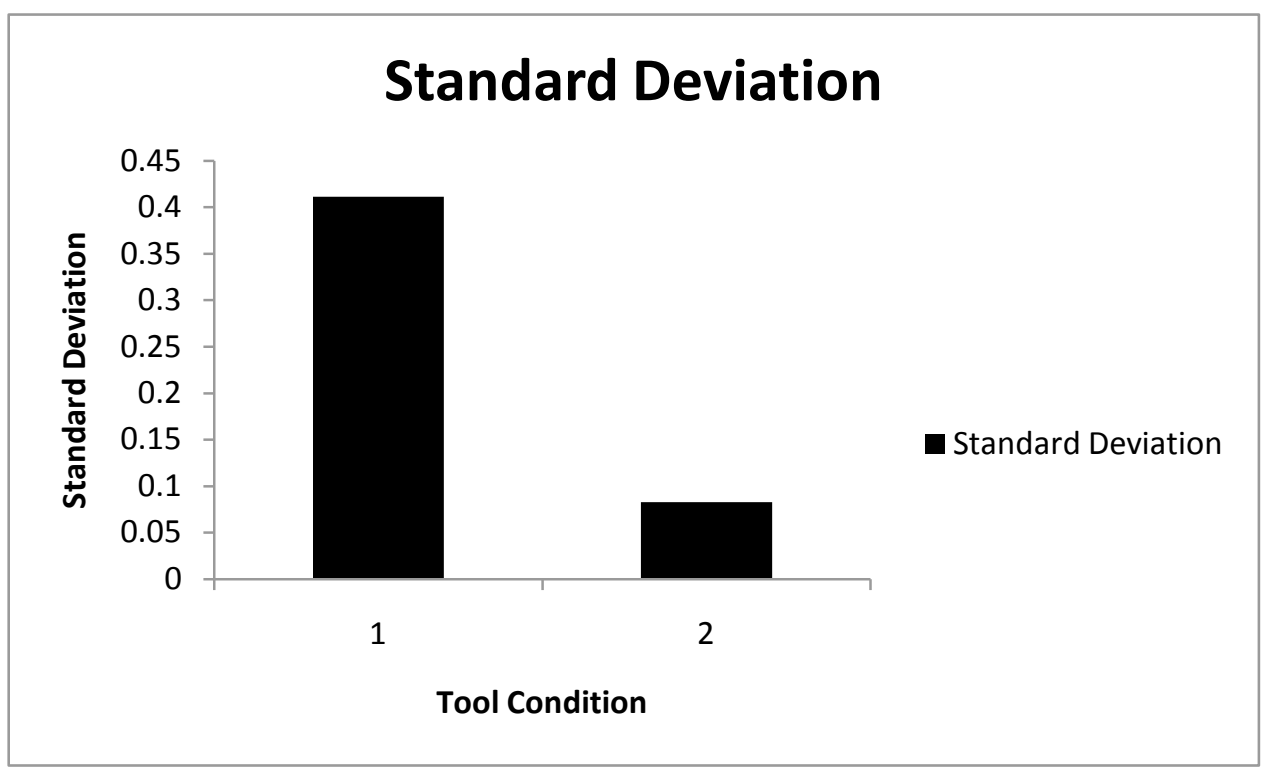

(a)

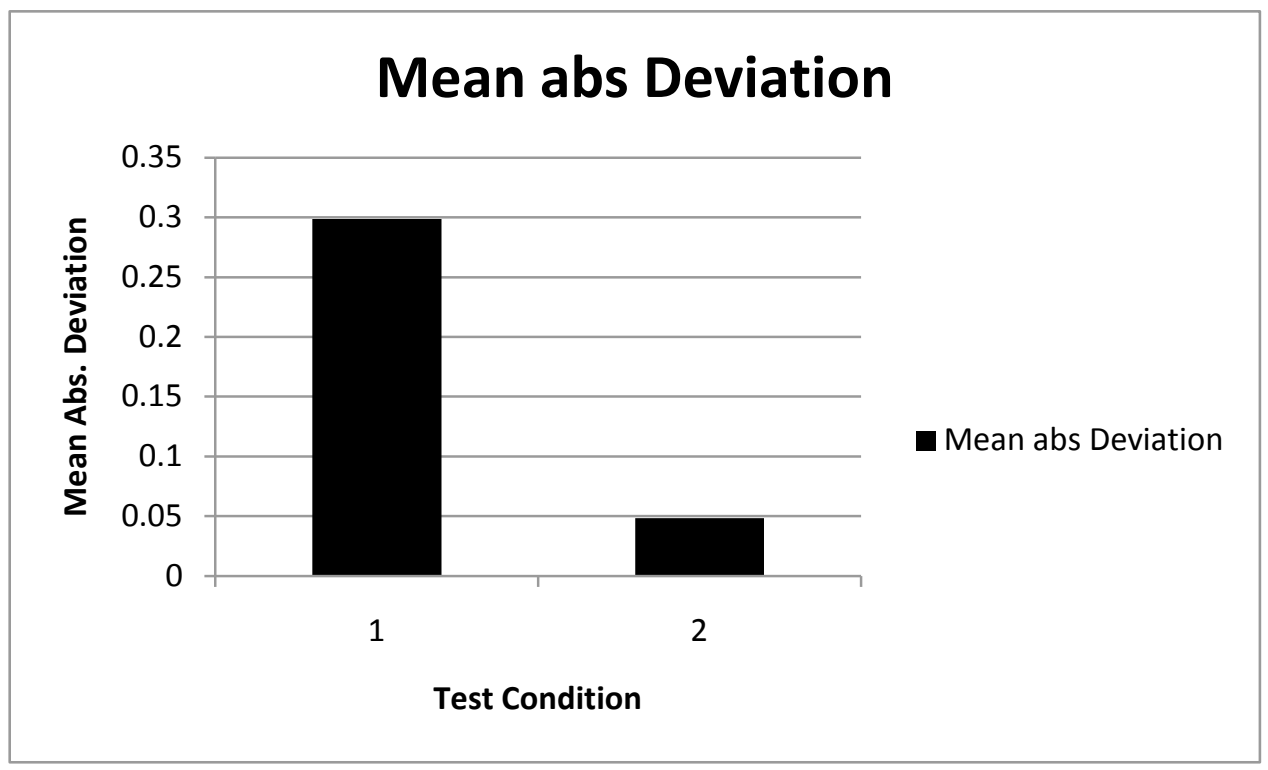

(b)

Figure 6.30 Comparison of statistical data between a the cut and hole imperfections: (a) Standard Deviation (b) Mean Abs Deviation 
To validate the results, the same experiment was conducted in two trails. The results of the two trails were compared to estimate the error in the percentage deviation. The error showed in Figure 6.31 concluded that that binary residue method could be successfully used to separate dissimilar imperfections in a weld joint.

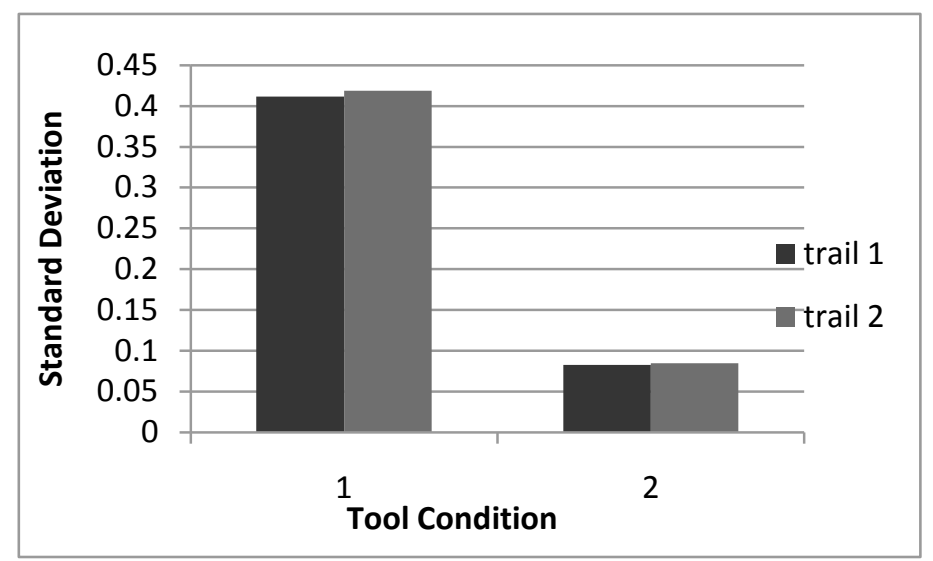

Figure 6.31 Error in SD values between different trails of the experimental analysis

\subsection{Brief Summary}

A complete analysis of the results obtained in Chapter Three of the dissertation was performed in this Chapter. It also investigated the effectiveness of piezoelectric actuators to sense the tool wear in and end mill tool using Lamb wave and SuRE techniques. The tool wear was classified into different wear levels. Finally the residue statistical data of the wavelet coefficients was used to separate dissimilar imperfections in welds. 


\section{CHAPTER 7}

\section{CONCLUSION AND FUTURE WORK}

The integrity of complex parts and welds was inspected by using the SuRE and Lamb wave methods in this work. The study started by characterizing the response of the piezoelectric materials since they were used as actuator and sensor for the tested SHM methods.

7.1 Characterization of Piezoelectric Materials and their Application:

Piezoelectric materials were used to investigate their electric generation characteristics. Their dynamic response was obtained with a spectrum analyzer. Two piezoelectric elements were used to construct energy scavengers. The frequency response of one of the piezoelectric elements was tested by exciting them. The natural frequency of the developed AA battery size scavenger was estimated at $1,135 \mathrm{~Hz}$ with a mass of $5.192 \mathrm{~g}$.

Energy scavengers were designed in rectangular and round profiles, and their performance was measured by using a digital oscilloscope. The round and rectangular energy scavengers generated an output of $250 \mu \mathrm{W}$ and $625 \mu \mathrm{W}$, respectively when they were excited at $50 \mathrm{~Hz}$ frequency. The study indicated that piezoelectric elements work effectively at higher frequencies. It was difficult to work with them when the mechanical vibration is less than $40 \mathrm{~Hz}$ frequency. 
7.2 Monitoring the Integrity of the Complex Geometries and Welded Parts

The integrity of the cutting tools with complex geometries and welded parts were inspected by using the Lamb wave and SuRE methods. Both methods used the piezoelectric elements for excitation and surface vibration monitoring. Sum of the squares of the differences of a reference signal and bi-orthogonal wavelet (Binlet) methods were used to interpret the data.

The wear of the cutting edges of a conventional tool and a micro end mill were evaluated by using two SHM methods. First, the propagation of the Lamb waves was monitored. The envelope of the received signal was obtained by using the stransformation. The height of the peaks of the envelopes at $20.4 \mathrm{kHz}$ and $90 \mathrm{kHz}$ frequencies correlated with the wear level of the tool. The shape of the envelope of the received signals was also changed with wear. Secondly, SuRE method was used to estimate the tool wear. The sum of the squares of the difference increased with the tool wear. The Lamb wave and SuRE methods complemented each other. Statistical analysis was conducted with the Lamb wave signal data using the bi-orthogonal wavelet (Binlet), to estimate the wear levels. They were derived from the acquired signal of the piezoelectric elements to estimate the different stages of the tool wear. The residual coefficients were used for interpretation of the signal. The wavelet coefficients increased with the tool wear. The standard deviation residues derived from the coefficients were correlated with the wear levels of the tested end mills.

The integrity of the welds was investigated by using both the SHM methods. Both of the methods identified the cuts and holes as soon as they were created on the 
weld, on the plate which has the actuator and sensor, and even on the plate which has no piezoelectric elements. The study indicated that the Lamb waves moved over the welds, and elastic behavior of the surface was affected as a whole. The residuals of the binlet analysis conducted using the Lamb wave data also identified the defects. Percentage variation in the statistical data indicated the type of the defect. The maximum difference of the means and the standard deviations were very small when the tests were repeated at the identical conditions (less than $0.3 \%$ ). However, the mean and SD significantly changed, when the weld or the parts were damaged.

\subsection{Future Work}

The following could be potential research topics in the near future:

1. Presently, Lamb wave and surface response data were analyzed separately to monitor the tool wear. Data analysis techniques like Neural Networks and Fuzzy Logic may be adopted to interpret the signals of both methods at the same time.

2. The discussed SHM methods may be used for other applications which have welds such as structures of the nuclear facilities. Also, SHM methods may be used for monitoring the wear and condition of the cutting tools. 


\section{LIST OF REFERENCES}

Abdukiram, T ., Nijima, K., and Takano, S., Design of bi-orthogonal wavelet filters using dyadic lifting scheme, Bulletin of Informatics and Cybernetics, Research Association of Statistical Sciences, Japan, 37 (2005) 123-136

Ali, N., and Haddad, R.A., Multi-resolution signal decomposition: transforms, sub bands, and wavelets, Second edition, Bell Communications Research (2001)

Altintas, Y., and Yellowley, I., In-process detection of tool breakage in milling using cutting force models, Journal of Engineering for Industry 111 (1988) 149-157

Altintas, Y., Yellowley, I., and Tlusty, J., The detection of tool breakage in milling operations, Journal of Engineering for Industry 110 (1988) 271-277

Bajaba, N. S., and Alnefaie, K. A., Multiple damage detection in structures using wavelet transforms, Emirates Journal for Engineering Research, 10 (2005) 1: 35-40

Baker, A., Rajic, N., and Davis, C., Towards a practical structural health monitoring for patched cracks, Composite Structures (2008), in press

Baptista, F., and Filho, J.V., The influence of the structural area on the performance of SHM systems based on electromagnetic Impedance, Proc. of $7^{\text {th }}$ International Workshop on Structural Health Monitoring, 1 (2009) 691-697

Beeby, S. P., Tudor, M. J., and White, N. M., Energy harvesting vibration sources for microsystems applications, Measurement Science and Technology, 17 (2006) 175-195

Bhalla, S., Gupta, A ., Bansal, S ., and Garg, T., ultra low-cost adaptations of electromechanical impedance technique for structural health monitoring, Journal of Intelligent Material Systems and Structures, 20 (2009) 8 :991-999

Castejon, M., Alegre, E., Barreiro, J., and Hernandez, L.K., On-line tool wear monitoring using geometric descriptors from digital images, International journal of Machine tools \& Manufacturing, 47(2007)1847-1853

Charnegie, D., Frequency tuning conducts for piezoelectric cantilever beams and plates for energy harvesting, M.S. Thesis, University of Pittsburgh, (2007)

Cooklev, T., Nishihara, A., and Sablatash, M., Regular orthonormal and bi-orthogonal wavelet filters, Signal Processing, 57(1997) 121-137

Cui, L., Zhai, B., and Zhang, T., Existence and design of bi-orthogonal matrix-valued wavelets, Nonlinear Analysis, 10 (2009) 2679-2687 
David, B. H., Rationalizing the coefficients of popular bi-orthogonal wavelet filters, IEEE Transactions on Circuits and Systems, 10(200) 6

Duhamel, P., and Vetterli, M., Fast Fourier tranforms: A tutorial review and a state of the art, Signal Processing, 19 (1990)4: 259-299

Dunn, C. T., Raghavan, A., and Kessler, SS., Analytical axisymmetric coupled piezoelasto dynamic models for guided Wave structural health monitoring, Proc. of $7^{\text {th }}$ International Workshop on Structural Health Monitoring, 1(2009)729-736

Dutoit, N.E., Wardle, B.L., and Kim, S.G., Design considerations for MEMS-Scale piezoelectric mechanical vibration energy harvesters, Integrated Ferroelectrics, 71 (2005) $21-160$

Ertunc, H.M., and Oysu, C., Drill wear monitoring using cutting force signals, Mechatronics, 14 (2004) 533-548

Erturk, A., Electromechanical modeling of piezoelectric energy harvesters, Ph.D. thesis, Virginia Polytechnic Institute and State University (2009)

Filho, J. V., Baptista, F. G., Inmann, D. J., Time-domainanalysis fo piezoelectric impedance -based structural health monitoring using multi-level wavelet decomposition, Mechanical systems and signal processing, in press

Genovese, L., Videau, B., Ospici, M., Deutsch, T., Goedecker, S. J., and Mehaut, F., Daubechies wavelets for high performance electronic structure calculations: The Big DFT Project, Academic des science, (2010), in press

Giurgiutiu, V., Zagrai, A., and Bao, J., Active sensors for health monitoring of aging aerospace structures, International journal of condition monitoring and diagnostic engineering management, (2001)

Giurgiutiu, V., Embeded NDE with piezoelectric wafer active sensors in aerospace applications, Journal of Materials, (2003)

Giurgiutiu, V., Lamb wave generation with piezoelectric wafer active sensors for structural health monitoring, Annual International Symposium on NDE for Health Monitoring and Diagnostics, San Diego (2002)

Giurgiutiu, V., Structural health monitoring with piezoelectric wafer active sensors, Academic Press, Elsevier, (2007)

Giurgiutiu, V., Zagrai, A., Bao, J., Damage Identification in Aging Aircraft structures with Piezoelectric Wafer Active Sensors, Journal of intelligent material systems and structures, 15(2004) 9-10:673-686 
Goldfarb M., and Jones, L.D., On the Efficiency of electric power generation with piezoelectric ceramic, ASME Journal of Dynamic Systems, Measurement and Control, 121 (1999) 566-571

Gong, W., Li, W., Shirakshi, T., Obikawa, T., An active method of monitoring tool wear states by impact diagnostic excitation, International Journal of Machine Tools \& Manufacture, 44 (2004) 847-854

Grabowska, J. ., Palacz, M., and Krawczuk, M., Damage identification by wavelet analysis, Mechanical Systems and Signal Processing, 22 (2008) 1623-1635

Gyuhae, P., Charles, R. F., Amanda, C. R., and Amy, N. R., Piezoelectric active sensor self-diagnostics using electrical admittance measurements, ASME Journal of Vibration and Acoustics, 128 (2006) 4: 469-476

Gyuhae, P., Charles, R. F., Francesco L., Stefano C., Performance assessment and validation of piezoelectric active-sensors in structural health monitoring, Smart Materials and Structures, 15 (2006) 16: 1673-1683

He, T.X., Bi-orthogonal spline type wavelets, International Journal of Computers \& Mathematics with Applications, 48 (2004) 1319-1334

Helge, P., Frans, F., and Martine, W., Durability and self-testing of sensor bonding used in structural health monitoring, EU Project meeting, AISHA, Leuven, Belgium (2007)

Hernandez-Salazar, C.D., Baltazar, A., Aranda-Sanchez, J. I., Damage detection in multi wire cables using continuous wavelet transform analysis of ultrasonic guided waves, Robotics and Automotive Mechanics Conference, (2009)

Huang, H., Pamphile, T., and Derriso, M., Directionality of Lamb wave displacement field generated by circular piezoelectric wafer actuators, Proc. of $7^{\text {th }}$ International Workshop on Structural Health Monitoring, 1 (2009) 1088-1095

Huang, H., Pamphile, T., and Derriso, M., The effect of actuator bending on Lamb wave displacement fields generated by a piezoelectric patch, Smart Mater. Struct. 17 (2008) 113

Inman, D., Grisso, B. L., and Sodano, H. A., Smart Materials in Structural Health Monitoring in Mechanical Vibration, CISM Courses and Lectures No. 488, Ed. by Isaac Elishakoff, SpringerWien, New York, (2007) 179-188

Kannan, E., Maxfield, B.W., and Krishnan B., SHM of pipes using torsional waves generated by in situ magnetostrictive tapes, Smart Mater. Struct. 16(2007)2505-2515 
Keinert, F., Biorthogonal wavelets for fast matrix computations, Mathematics Subject Classification (1996)

Kerkira, G. R., Shinde, V., Schulz, M., Ghoshal, A., Sundaresan, M., Allemang, R., Damage localization in composite and metallic structures using a structural neural system and simulated acoustic emissions, mechanical systems and signal processing, 21 (2007) 280-297

Kessler, S.S., and Raghavan, A., Vector -based damage localization for anisotropic composite laminates, Proc. of $7^{\text {th }}$ International Workshop on Structural Health Monitoring, 2 (2009) 1328-1335

Khare, A., Khare, M., Jeong, Y., Kim, H., and Jeon, M., Despepeckling of medical ultrasound images using daubechies complex wavelet transform, Signal Processing, 90 (2010) 428-439

Kim, B.H., Park, T., and Voyiadjis, G.Z., International Journal of Solids and Structures, 43 (2006) 14-15:4238-4257

Kim, S., Clark, W.W., Wang, Q.M., Piezoelectric energy harvesting with a clamped circular plate:Experimental study, Journal of Intelligent Materials Systems and Structures, 16 (2005) 847-854

Kirby, J.F., Which wavelet best produces the Fourier power spectrum, Computers \& Geosciences, 31 (2005) 846-864

Kivio, J., and Andersson, P.H., Tool breakage monitoring in a multi spindle drilling operation, XVII IMEKO world congress, Metrology in the $3^{\text {rd }}$ Millennium, 2003, Croatia

Konstantinidis, G., Drinkwater, B. W., and Wilcox, P. D., The temperature stability of guided wave structural health monitoring systems, Smart Materials and Structures, 15 (2006) 967-976

Kulesh, M., Holschneider, M., and Diallo, M.S., Geophysical wavelet library: Applications of the continuous wavelet transform to the polarization and dispersion analysis of signals, Computers \& Geo sciences, 34 (2008) 1732-1752

Kunpeng, Z., San, W. Y., and Soon, H. G., Wavelet analysis of sensor signals for tool condition monitoring: A review and some new results, Machine tools and manufacture, 49 (2009) $537-553$

Lamb, H., on waves in elastic plates, Proc. Roy. Soc. London, A 93 (1917) 114-128 
Sejdić,E., Djurović, I., and Stanković, L., Fractional Fourier Transform as a signal processing tool: An overview of recent developments, Signal Processing, 91(2011)6: $1351-1369$

Lee, B. C., and Staszewski, W, J., Sensor location studies for damage detection with Lamb waves, Smart Materials and Structures, 16(2007) 399-408

Lee, B. C., and Staszewski, W. J., Sensor location studies for damage detection with Lamb waves, Smart Materials and Structures, 16(2007) 399-408

Lee, B.C., and Staszewski, W.J., Modeling of Lamb waves for damage detection in metallic structures: Part II. Wave interaction damage, Smart Materials and Structures, 1726 (2003) 67509-9/12 (2003) 815-824

Lee, B.C., and Staszewski, W.J., modeling of Lamb waves for damage detection in metallic structures: Part II. Wave interaction damage, Smart Materials and Structures, 1726(2003)67509-9/12(2003)815-824

Lemistre, M., and Balageas, Structural health monitoring system based on diffracted Lamb wave analysis by multi resolution processing, Smart Mater. Struct. 10(2001) 504

Lemistre, M., Balageas, D., Structural health monitoring system based on diffracted Lamb wave analysis by multiresolution processing, Smart Materials and Structures, 10 (2001) 504-511

Li, X., A brief review: acoustic emission method for tool wear monitoring during turning, International journal of Machine tools \& Manufacturing, 42 (2002) 157-165

Li, X., Dong, S., and Yuan, Z., Discrete wavelet transform for tool breakage monitoring, Machine Tools and Manufacture, 39 (1999) 1935-1944

Lim, Y.Y., Suresh B., and Soh, C.K., Structural identification and damage diagnosis using self-sensing Piezo- impedance transducers, Smart Materials and Structures, 15 (2006) 987-995

Lin, B., and Giurgiutiu, V., Modeling and testing of PZT and PVDF piezoelectric wafer active sensors, Smart Materials and Structure, 15 (2006) 1085-1093

Lin, S.C., and Lin, R. J., Tool wear monitoring in face milling using force signals, Wear, 198 (1998)136-142

Lipinski, P., and Yatsymirskyy, M., Efficient 1D and 2D daubechies wavelet transforms with application to signal processing, ICANNGA, (2007) 391-398 
Liu, L., and Yuan, F.,G., Active damaze localization for plate like structures using wireless sensors and distributed algorithm, Smart Materials and Structures, 17 (2008) 5: $\mathrm{xx}-\mathrm{xx}$

Loosvelt, M., and Lasaygues, P., A wavelet based processing method for simultaneously determining ultrasonic velocity and material thickness, Ultrasonics, (2010) 055022

Lotfollahi-Yaghin, M. A., and Hesari, M. A., Using wavelet analysis in crack detection at the arch concrete dam under frequency analysis with FEM, 3 (2008) 4:691-704

Lu, F., Lee, H.P., and Lim, S.P., Modeling and Analysis of Micro Piezoelectric Power Generators for Micro-Electromechanical-Systems Applications, Smart Materials and Structures, 13 (2004) 57-63

Lynch, J.P., Linear classification of system poles for structural damage detection using piezoelectric active sensors, SPIE $11^{\text {th }}$ annual international symposium on smart structures and materials, San Diego (2004)14-18

Mallat, .G., A theory for multi resolution signal decomposition : The wavelet representation, IEEE transactions on Pattern analysis and machine Intelligence, 11 (1989) 7:674-693

Mehrjoo, M., Khaji, N., Moharrami, H., Bahreininejad, A., Damage detection of truss bridge joints using artificial neural networks, Expert Systems with Applications, 35 (2008) 1122-1131

Minazara, E.., Vasic, D., Costa, F., and Poulin, G., Piezoelectric diaphragm for vibration energy harvesting, Proc. Of Ultrasonics international, 44 (2006) 699-703

Mossi, K., Green, C., Ounaies, Z., and Hughes, E., Harvesting energy using a thin unimorph prestressed bender: geometrical effects, Journal of Intelligent Material Systems and Structures, 16 (2005) 249-261

Nair, K. K., and Kiremidjian, A. S., A damage detection algorithm using the morlet wavelet transform, Stanford University (2009)

Niezrecki, C., Brei, D., Balakrishnan, S., and Moskalik, A., Piezoelectric Actuation: State of the Art, Shock and Vibration Digest, 33 (2001) 269-280

Ozmen, B., and Ozkaramanli, H., Complex linear phase biorthogonal filterbanks with approximately analytic wavelets, Signal Processing, 89 (2009) 599-604

Park, S., Park, G., Yun, C., and Farrar, C.R., Sensor self-diagnosis using a modified impedance model for active sensing -based structural health monitoring, Structural Health Monitoring, (2009) 
Park, S., Yun, C., and Roh, Y., Damage diagnostics on a welded zone of a steel truss member using and active sensing network system, NDT \&E International, 40 (2007) 7176

Park, G., Cudney, H. H., Inman, D.J., An integrated health monitoring technique using structural impedance sensors, Journal of Intelligent Material Systems and Structures, 11 (2000) 6:448-455.

Park, G., H Sohn, Farrar, C. R., Inman, D. J., "Overview of Piezoelectric ImpedanceBased Health Monitoring and Path Forward", The Shock and Vibration Digest, 35 (2003) 6: $451-463$

Patsias, S., Sataszewski, W. J., Damage detection using optical measurements and wavelets, Structural Health Monitoring, 1 (2002) 1: 5-22

Peairs, D. M., Park, G., Inman, D J., Improving accessibility of the impedance-based structural health monitoring method, Journal of Intelligent Material Systems and Structures, 15 (2004) 2: 129-2139

Pei, S.C., Yeh, M.H., Twp dimensional discrete fractional Fourier Transform, Signal Processing, 67 (1998)1:99-108

Pena, B., Armendeni, G., Rivero, A., Lopez de Lacalle, L.N., Monitoring of drilling for burr detection using spindle torque, International Journal of Machine tools \& Manufacture, 45 (2005) 1614-1621

Pfeiffer, H., Structural health monitoring of slat tracks using transient ultrasonic waves, EU Project on Aircraft Integrated Structural Health Assessment, (2007)

Prickett, P.W., Johns, C., An overview of approaches to end milling tool monitoring, International journal of Machine tolls \& Manufacture, 39 (1999) 105-122

Raghavan, A., Cesnik, C.E.S., Finite-dimensional piezoelectric transducer modeling for guided wave based structural health monitoring, Smart Mater. Struct. 14 (2005) 14481461

Rajic, N., Galea, S.C., and Chiu, W.K., Autonomous detection of crack initiation using surface -mounted piezo transducers, Smart Mater. Struct. 11 (2002) 107-114

Rodrigo, A., Munoz, S., Roberto A., and Lopez, A., Structural health monitoring of composite structural joints using embedded fiber Bragg grating strain sensors, Composite Structures, 89 (2009) 224-234 
Roemer, M.J., Kacprzynski, G.J., Nwadiogbu, E.O., Bloor, G., Development of diagnostic and prognostic technologies for aerospace health management applications, IEEE, (2001)

Rout, S., Orthogonal Vs. Biorthogonal wavelets, Master's thesis in electrical engineering, Virginia polytechnic Institute and state university, (2003)

Rucka, M., Wilde, K., Application of continuous wavelet transform in vibration based damage detection method for beams and plates, Journal of Sound and Vibration, 297 (2006) 536-550

Rutherford, A.C., Gyuhae, P, and Farrar, C.R., Non-linear feature identifications based on self-sensing impedance measurements for structural health assessment, Mechanical Systems and Signal Processing, 21 (2007) 322-333

Santoni-Bottai,G., and Giurgiutiu, V., Shear lag solutions for solid state actuators bonded to structures in SHM applications, Proc. of $7^{\text {th }}$ International Workshop on Structural Health Monitoring, 1 (2009) 713-720

Singh, G., Monitoring the health of plates with simultaneous application of Lamb waves and surface response to excitation approaches, M.S. Thesis, Florida International University (2010)

Sirohi J., and Chopra, I., Fundamental Understanding of Piezoelectric Strain Sensors, Journal of Intelligent Material Systems and Structures, 11 (2000) 246-257

Sodano, H. A., Macro-Fiber Composites for Sensing, Actuation and Power Generation, M.S. Thesis, Virginia Polytechnic Institute and State University, (2003)

Sodano, H.A., Inman, D.J., and Park, G., Comparison of piezoelectric energy harvesting devices for recharging batteries, Journal of Intelligent Material Systems and Structures, 16 (2005) 799-807

Sodano, H.A., Park, G., and Inman, D.J., Estimation of electric charge output for piezoelectric energy harvesting, Strain, 40 (2004) 49-58

Sohn, H., and Lee, S. J., Time reversal based piezoelectric transducer self-diagnosis under varying structural conditions, Proc. of $7^{\text {th }}$ International Workshop on Structural Health Monitoring, 1 (2009) 854-861

Stephane M., A wavelet tour of signal processing, second edition, Book (1998)

Stockwell, R. G, Mansinha, L., and Lowe, R. P., Localization of complez sprectrum: the S transform, IEEE transactions on Signal Processing, 44 (1996) 4: 998-1001 
Stoic, A., and Raos, P., Tool condition monitoring system, $12^{\text {th }}$ International science conference achievements in Mechanical \& Materials Engineering, (2003)

Strang, G., and Nguyen, T., wavelets and filter banks, Wellesley Cambridge Press, (1996)

$\mathrm{Su}, \mathrm{Z}$., Ye, L., and Lu, Y., Guided Lamb waves for identification of damage in composite structures: A review, Journal of Sound and Vibrations 295 (2006) 753-780

Tan, K. S., Guo, N., Wong, B.S., and Tui, C. G., Comparision of Lamb waves and pulse echo in detection of near surface defects in laminate plates, NDT \&E International, 28 (1995) 215-223

Tansel, I.N., Demetgul, M., Leon, R.A., Yenilmez, A., and Yapici, A., Design of energy scavengers of health monitoring systems by using GONNS, Sensors and Materials, 21 (2009)3:141- 154

Tansel, I.N., Makdeci, C., and Mclaughlin, C., Detection of tool failure in end milling with wavelet transforms and neural networks (WTNN), International Journal of Machine Tools and Manufacture 35 (1995) 1137-1147

Tansel, I.N., Leonard, R., Yenmilmez, A., Yapici, A., Korla, S., Demetgul, M., Powering next generation structural health monitoring sensors with energy scavengers, ISHM conference, 2008

Tao, H., and Moorhead, R.J., Progressive transmission of scientific data using biorthogonal wavelet transform, IEEE Journal (1994)

Tay, B. H., Family of binary coefficients of bi-orthogonal wavelet filters, International symposium on circuits and systems, Geneva (2000)

Torrence, C., Compo, G.P., A Practical Guide to wavelet Analysis, Bulletin of the American meteorological society, (1998)

Tseng, K.K., and Wang, L., Structural damage identification for thin plates using smart piezoelectric transducers, Computer Methods in Applied Mechanics and Engineering, 194 (2005) 3192-3209

Twiefel, J., Richter, B., Hemsel, T., and Wallaschek, J., Model-based design of piezoelectric energy harvesting systems, Proc. of SPIE., 6169 (2006), 09.1-09.10

Verl, A., Eisel, U., Walther, M., Maier, D., Sensor less automated condition monitoring for the control of the predictive maintenance of machine tools, CIRP AnnalsManufacturing Technology,58 (2009)375-378 
Vetteri, M., and Herley, C., wavelets and filter banks: theory and design, Signal Processing, 40 (1992) 9

Vonende, S., and Lammering, R., Piezoelectrically generated Lamb waves in elastic and viscoelastic plates-analysis and experimental verification, Proc. of $7^{\text {th }}$ International Workshop on Structural Health Monitoring, 1 (2009) 675-682

Wang, X., and Tansel, I. N., Modeling the propagation of Lamb waves using a genetic algorithm and S-Transformation, Structural health monitoring, 6 (2007) 25-37

$\mathrm{Xu}, \mathrm{B} ., \mathrm{Yu}, \mathrm{L}$., and Giurgiutiu, V., Advanced methods for time of flight estimation with application to Lamb wave structural health monitoring, Proc. of $7^{\text {th }}$ International Workshop on Structural Health Monitoring, 2 (2009) 1210-1217

$\mathrm{Xu}$, B., Giurgiutiu, V., A Low-Cost and Field Portable Electromechanical (E/M) Impedance Analyzer for Active Structural Health Monitoring, Structural Health Monitoring, Edited by Fu-Kuo Chang, (2005): 634-644

Yan, Y.J., and Yam, L.H., online detection of crack damage in composite plates using embedded piezoelectric actuators/sensors and wavelet analysis, Composite Structures, 58 (2002) 29-38

Yenilmez, A., Yapici, A., Tansel, I. N., Martin, S. A., Pereira, C. M., and Roth, L. E., Review of progress in quantitative nondestructive evaluation, edited by Drs. D.O. Thompson and D.E. Chimenti, Design of Energy Scavengers with the Help of Finite Element Packages, AIP Press, 894 (2007) 957-966

Zhao, X., Qian, T., Mei, G., Kwan, C., Zane, R., Walsh, C., Paing, T., Popovic, Z., Active health monitoring of an aircraft wing with an embedded piezoelectric sensor: II wireless approaches, Smart Mater. Struct., 16 (2007) 1218-1225

Zhu, X.Q., Law, S.S., Wavelet based crack identification of bridge beam from operational deflection time history, International Journal of Solids and Structures, 43 (2006) 2299-2317 
VITA

\section{SRIKANTH KORLA}

\section{EDUCATION}

2001

2004

2011

\section{EXPERIENCE}

2003-2004

2004-2007

2007-2011
B.E in Mechanical Engineering Andhra University Visakhapatnam, India

$\mathrm{ME}$ in $\mathrm{CAD} / \mathrm{CAM}$ Andhra University Visakhapatnam, India

Doctoral Candidate in Mechanical Engineering Florida International University Miami, USA

Lecturer in Mechanical Engineering JNT University Kakinada Kakinada, India

Assistant Professor in Mechanical Engineering GITAM University

Visakhapatnam, India

Graduate Teaching Assistant

Department of Mechanical and Materials

Engineering, Florida International University

\section{PUBLICATIONS}

Korla, S., Leon, RA., Tansel, IN., Yenilmez, A., Yapici, A., Demetgul, M., Design and testing of an efficient and compact Piezo-electric battery for micro power generation Journal of Microelectronics, 42 (2011) 265-270.

Tansel, I.N., Grisso, B.L., Singh, G., Singh, G., Korla, S., Duran, R., Salvino, L.W., Wear estimation by testing the elastic behavior of tool surface, International Journal of Machine Tools and Manufacture, In Press, Available online 12 June 2011.

Korla, S., Shinde, P., Ajrawat, T., Design and Development of a Flipping Biped Robot, ASME early Technical Journal, 7, (2008) 1.1-1.5 
Tansel, I, N. Singh G., and Korla, S., Grisso, and Salvino, L. W., Characterization and compaction of Lamb wave data using a combination of $\mathrm{S}$ and wavelet transformations, Proc. SPIE 7982, San Diego, California, USA, 2011.

Tansel, I N., Grisso B L., Singh, G., Korla, S., Salvino, L W., Uragan, v., Monitoring the integrity of machine assemblies by using surface response to excitation (SuRE) approach, RAST 2011 : 5th International Conference on Recent Advances in Space Technologies, Istanbul, Turkey, June 2011.

Tansel, I N., Singh, G., Singh, G., Korla, G., Li. M., Genetically Optimized Neural Network Systems (GONNS) Applications for Unmanned Vehicles, UVW conference, 2010.

Tansel, I.N., Li, M., Singh, G., Singh, G., Korla, S., Monitoring the health of unmanned aerial vehicles, UVW Conference, 2010.

Demetgul, M., Korla, S., Tansel, I.N., Hybrid driver concept for powering Ornithopters, Proc. of $47^{\text {th }}$ AIAA Aerospace meeting, Orlando, 2009.

Tansel, I.N., Korla S., and Demetgul M., Degradation of Signal Quality of Structural Health Monitoring Systems and Fault Tolerant Designs for Life Time Operation, presented along with ISHM Conference, Cincinnati, 2009.

Tansel, I. N., Leonard, R ., Yenilmez, A., Yapaci, A ., Korla, S., and Demetgul, M., Powering Next generation structural health monitoring sensors with energy scavengers Presented at Integrated Health Monitoring systems, Cincinnati, 2008.

Korla, S., and Ramji, K, Time scope study of dynamic system primitives, presented at Andhra University, Visakhapatnam, India, 2005.

Korla, S., Ramji, K., Simulation of rear wheels of a driveline when a vehicle takes a turn, Presented at JNT University, Kakinada, India, 2004.

Korla, S., Ramarao, S., Sound Pollution and its control, Presented at IMPETUS 2000, Gayatri Vidya Parishad college of Engineering, Visakhapatnam, India, 2000. 